\title{
Infection Prevention and Control Guideline for Cystic Fibrosis: 2013 Update
}

\author{
Lisa Saiman, MD, MPH; ${ }^{1, a}$ Jane D. Siegel, MD; ;,a John J. LiPuma, MD; ${ }^{3, a}$ Rebekah F. Brown, MD; ${ }^{4}$ \\ Elizabeth A. Bryson, RN, MSN, PPCN-BC, CS; ${ }^{5}$ Mary Jo Chambers, LCSW, MSW; ${ }^{6}$ Veronica S. Downer, RN; ${ }^{7}$ \\ Jill Fliege, APRN; ${ }^{8}$ Leslie A. Hazle, MS, RN, CPN, CPHQ; ${ }^{9}$ Manu Jain, MD, MS; ${ }^{10}$ Bruce C. Marshall, MD, MMM; ${ }^{11}$ \\ Catherine O’Malley, RRT-NPS, AS; ${ }^{12}$ Suzanne R. Pattee, JD; ${ }^{13}$ Gail Potter-Bynoe, BS; ${ }^{14}$ Siobhan Reid $;{ }^{15}$ \\ Karen A. Robinson, PhD $;{ }^{16}$ Kathryn A. Sabadosa, MPH $;{ }^{17}$ H. Joel Schmidt, MD $;{ }^{18}$ \\ Elizabeth Tullis, MD, FRCPC $;{ }^{19}$ Jennifer Webber; ${ }^{20}$ David J. Weber, MD, MPH ${ }^{21, b}$
}

\section{TABLE OF CONTENTS}

\section{EXecutive SumMary}

INTRODUCTION

Methods for Document Development

Committee Structure

Systematic Review Process

Results of the Systematic Review

Process for Inclusion of Recommendations

External Review

Updated Recommendations FOR IP\&C in CF

I. Core Recommendations

II. Recommendations for Microbiology and Molecular Epidemiology

III. Recommendations for CF Clinics and Other Ambulatory Care Settings

IV. Recommendations for Inpatient Settings

V. Recommendations for Nonhealthcare Settings

VI. Recommendations for Healthcare Personnel with CF

VII. Recommendations for Psychosocial and Medical Impact of IP\&C
Background Information Supporting the RECOMMENDATIONS

I. CF Microbiology and Molecular Typing

I.A. General Microbiology Methods

I.B. Molecular Typing Methodologies

I.C. Epidemiology of CF Pathogens

I.C.1. Overview

I.C.2. Burkholderia spp.

I.C.3. Other Gram-Negative Species

I.C.4. Small Colony Variant (SCV) S. aureus

I.C.5. Nontuberculous Mycobacteria

I.C.6. Aspergillus spp.

I.D. Surveillance Strategies for CF Pathogens

II. Routes of Transmission of CF Pathogens

II.A. Contact and Droplet Transmission

II.B. Classic View of Droplet Transmission

II.C. Emerging View of Droplet Transmission

II.D. Potential Role of Droplet Nuclei

II.E. Paradigm for Transmission of Respiratory Pathogens

Affiliations: 1. Department of Pediatrics, Columbia University Medical Center, New York, New York; and Department of Infection Prevention and Control, NewYork-Presbyterian Hospital, New York, New York; 2. Department of Pediatrics, University of Texas Southwestern Medical Center, Dallas, Texas; and Children's Medical Center, Dallas, Texas; 3. Department of Pediatrics and Communicable Diseases, University of Michigan Medical School, Ann Arbor, Michigan; and Department of Epidemiology, University of Michigan School of Public Health, Ann Arbor, Michigan; 4. Department of Pediatrics, Vanderbilt University Medical Center, Nashville, Tennessee; 5. Department of Pediatrics, Akron Children's Hospital, Akron, Ohio; 6. Department of Social Work, Arkansas Children's Hospital, Little Rock, Arkansas; 7. University of Michigan Hospital, Ann Arbor, Michigan; 8. Pulmonary Division, Nebraska Medical Center, Omaha, Nebraska; 9. Current affiliation: Director, Clinical Operations and Performance Improvement, Spectrum Health Medical Group, Grand Rapids, Michigan; 10. Department of Medicine, Feinberg School of Medicine, Northwestern University, Chicago, Illinois; 11. Medical Department, Cystic Fibrosis Foundation, Bethesda, Maryland; 12. Department of Pediatrics, Ann and Robert H. Lurie Children's Hospital of Chicago, Chicago, Illinois; 13. Adult with cystic fibrosis, Silver Spring, Maryland; 14. Department of Infection Prevention and Control, Boston Children's Hospital, Boston, Massachusetts; 15. Parent; 16. Johns Hopkins University, Baltimore, Maryland; 17. Dartmouth Institute for Health Policy and Clinical Practice, Geisel School of Medicine at Dartmouth, Lebanon, New Hampshire; 18. Department of Pediatrics, Children's Hospital of Richmond at Virginia Commonwealth University, Richmond, Virginia; 19. Department of Medicine, University of Toronto, and Keenan Research Centre of Li Ka Shing Knowledge Institute, St. Michael's Hospital, Toronto, Canada; 20. Parent; 21. Departments of Medicine and Pediatrics, University of North Carolina at Chapel Hill Medical School, Chapel Hill, North Carolina; a. Co-chairs of the Infection Prevention and Control Guideline for Cystic Fibrosis Committee; b. The list of authors represents the entire Infection Prevention and Control Guideline for Cystic Fibrosis Committee.

Received March 3, 2014; accepted March 5, 2014; electronically published July 1, 2014. Infect Control Hosp Epidemiol 2014;35(S1):S1-S67

(C) 2014 by The Society for Healthcare Epidemiology of America and The Cystic Fibrosis Foundation. All rights reserved. 0899-823X/2014/3508S1-0001\$15.00. DOI: $10.1086 / 676882$ 
III. Potential Sources of CF Pathogens

III.A. Person-to-Person Transmission among People with CF

III.A.1. Burkholderia spp.

III.A.2. P. aeruginosa

III.A.3. Other Gram-Negative Bacteria

III.A.4. S. aureus

III.A.5. Nontuberculous Mycobacteria

III.B. Acquisition from People without CF

III.B.1. P. aeruginosa and Burkholderia spp.

III.B.2. S. aureus

III.B.3. Respiratory Viruses

III.C. Acquisition from Animals

III.D. Acquisition from the Inanimate Environment

III.D.1. Nonhealthcare Sources: Soil, Organic Matter, and Water

III.D.2. Healthcare Sources: Water, Surfaces, Equipment, Air, and Contaminated Products

III.D.3. Construction and Renovation

IV. Strategies to Reduce Transmission and Acquisition of CF Pathogens

IV.A. Overview

IV.B. Education Strategies

IV.C. Hand Hygiene

IV.D. Personal Protective Equipment (PPE)

IV.D.1. Gowns and Gloves

IV.D.2. Masks, Eye Protection, and Respirators

IV.E. Environmental Cleaning and Disinfection

IV.E.1. Overview

IV.E.2. Healthcare Facilities: Respiratory Therapy, Nebulizers, and Diagnostic Equipment

IV.E.3. Nonhealthcare Settings: Nebulizers

IV.F. Strategies for CF Clinics

IV.F.1. Cohort Segregation versus All-Patient Separation

IV.F.2. CF Clinic Logistics

IV.F.3. Hand Hygiene

IV.F.4. Mask Use by People with CF

IV.F.5. Gown and Glove Use by Healthcare Personnel

IV.F.6. Pulmonary Function Testing

IV.F.7. Environmental Practices

IV.F.8. Use of Restrooms

IV.G. Hospital Room Placement and Transmission-Based Precautions

IV.H. Construction and Renovation

IV.I. Nonhealthcare Settings

IV.I.1. Camps and Educational Retreats

IV.I.2. Indoor Events

IV.I.3. Outdoor Events

IV.I.4. Schools

IV.I.5. Prevention of Viral Transmission and Immunizations

V. Healthcare Personnel with CF

VI. Psychosocial and Medical Impact of Transmission-Based Isolation Precautions

VII. Challenges to Implementation of IP\&C Recommendations
Research Agenda

REFERENCES

TABLES

Table 1. Infection Prevention and Control Guidelines Published since 2003

Table 2. Population, Intervention, Comparison, and Outcome (PICO) Clinical Questions Developed for the Guideline for Infection Prevention and Control in Cystic Fibrosis (CF): 2013 Update

Table 3. Grading Systems Used in the Updated Infection Prevention and Control (IP\&C) Guidelines for Cystic Fibrosis (CF), 2013

Table 4. Burkholderia cepacia Complex

Table 5. Modes of Transmission of Potential Pathogens in Cystic Fibrosis

Table 6. Relative Frequency of Shared Strains of Different Cystic Fibrosis Pathogens

Table 7. Examples of Opportunities for Hand Hygiene by Healthcare Personnel, People with Cystic Fibrosis (CF), and Families

Table 8. Use of Personal Protective Equipment by Healthcare Personnel, People with Cystic Fibrosis (CF), and Families

Table 9. Strategies to Enhance the Effectiveness of Environmental Cleaning in Ambulatory and Inpatient Settings for People with Cystic Fibrosis (CF)

Table 10. Infection Prevention and Control Strategies for Cystic Fibrosis (CF) Clinics to Minimize the Risk of Transmission of Potential Pathogens within 6 Feet (2 Meters)

Table 11. Possible Strategies to Minimize the Adverse Psychosocial Impact of Isolation Precautions among People with Cystic Fibrosis (CF)

Table 12. Knowledge, Attitudes, and Practice Barriers Related to Implementing the Infection Prevention and Control Guideline in Cystic Fibrosis (CF)

Table 13. Strategies to Enhance Implementation of the Infection Prevention and Control Guideline among Healthcare Personnel, People with Cystic Fibrosis $(\mathrm{CF})$, and Families of People with CF

\section{Figures}

Figure 1. Age-Specific Prevalences of Respiratory Organisms, 2012

Figure 2. Respiratory Organism Prevalences, 1988-2012

\section{EXECUTIVE SUMMARY}

\section{INTRODUCTION}

The 2013 Infection Prevention and Control (IP\&C) Guideline for Cystic Fibrosis (CF) was commissioned by the CF Foundation as an update of the 2003 Infection Control Guideline for $\mathrm{CF}^{1}$ During the past decade, new knowledge and new 
challenges provided the following rationale to develop updated IP\&C strategies for this unique population:

1. The need to integrate relevant recommendations from evidence-based guidelines published since 2003 into IPঊC practices for $C F$. These included guidelines from the Centers for Disease Control and Prevention (CDC)/Healthcare Infection Control Practices Advisory Committee (HICPAC), the World Health Organization (WHO), and key professional societies, including the Infectious Diseases Society of America (IDSA) and the Society for Healthcare Epidemiology of America (SHEA). During the past decade, new evidence has led to a renewed emphasis on source containment of potential pathogens and the role played by the contaminated healthcare environment in the transmission of infectious agents. Furthermore, an increased understanding of the importance of the application of implementation science, monitoring adherence, and feedback principles has been shown to increase the effectiveness of IP\&C guideline recommendations.

2. Experience with emerging pathogens in the non-CF population has expanded our understanding of droplet transmission of respiratory pathogens and can inform IPঊC strategies for $C F$. These pathogens include severe acute respiratory syndrome coronavirus and the 2009 influenza A H1N1. Lessons learned about preventing transmission of methicillinresistant Staphylococcus aureus (MRSA) and multidrug-resistant gram-negative pathogens in non-CF patient populations also can inform IP\&C strategies for CF.

3. As the use of molecular technologies increased throughout the past decade, there is an improved understanding of the epidemiology of newer CF pathogens that are increasing in prevalence and are associated with increased morbidity and mortality. Such pathogens include MRSA, Mycobacterium abscessus, new species in the Burkholderia cepacia complex (eg, Burkholderia dolosa), and epidemic clones of Pseudomonas aeruginosa (eg, the Liverpool epidemic strain).

\section{Methods for Document Development}

An interdisciplinary committee of healthcare personnel with expertise in CF, 3 parents of children with CF, and an adult with $\mathrm{CF}$ determined the scope of the guideline, reviewed the evidence (including that from a systematic review), and developed and voted anonymously on specific recommendations. Whenever appropriate, this guideline has integrated relevant recommendations from the 2003 Infection Control Guideline for CF and from other existing IP\&C guidelines.

The focus of the updated guideline is to provide recommendations to prevent individuals with CF from transmitting and/or acquiring respiratory tract pathogens from others with $\mathrm{CF}$ in ambulatory care and inpatient settings. Recommendations for nonhealthcare settings represent efforts to respond to questions and concerns voiced by people with $\mathrm{CF}$ and their caregivers and, thus, are intended to educate the CF community about potential risks and to help people with CF and their families and friends make informed choices in their personal lives. Recommendations for nonhealthcare settings are not intended to be enforced by healthcare personnel.

This guideline is intended for use by all healthcare personnel involved with the care of people with CF and the IP\&C teams that support CF care centers in the United States. The recommendations for healthcare settings apply to inpatient settings, CF clinics and other ambulatory care areas, diagnostic and therapeutic areas, and all clinical research activities. Successful and consistent implementation of IP\&C practices must include the ongoing participation of people with $\mathrm{CF}$ and their families as well as auditing the IP\&C practices of healthcare personnel and feedback about their performance. The goal of this guideline is to reduce substantially the risk of transmission and acquisition of CF pathogens, while recognizing that the risk is unlikely to reach zero.

A draft of the guideline was made available to the CF and IP\&C communities for review, and all comments were considered by the committee. This guideline was reviewed and endorsed by SHEA and by the Association for Professionals in Infection Control (APIC).

\section{RECOMMENDATIONS}

The recommendations are divided into 7 sections.

I. Core recommendations, intended for all people with $\mathrm{CF}$ (including following lung or liver transplantation) in all settings

II. Recommendations for microbiology and molecular epidemiology

III. Recommendations for CF clinics and other ambulatory settings

IV. Recommendations for inpatient settings

V. Recommendations for nonhealthcare settings

VI. Recommendations for healthcare personnel with CF

VII. Recommendations regarding the psychosocial and medical impact of IP\&C

To facilitate use of the guideline, the relevant sections of "Background Information Supporting the Recommendations" (Sections I-III) and strategies to reduce transmission and acquisition of pathogens (Sections $I V-V I I)$ are provided with each recommendation. The recommendations emphasize that healthcare personnel, people with CF, and their family and friends receive education about IP\&C that fosters understanding of the rationale for the recommendations.

The recommendations highlight the importance of partnering with local IP\&C teams to facilitate implementation and the use of existing audit and feedback tools to monitor adherence to IP\&C practices. The recommendations emphasize source containment of the respiratory secretions of people with CF, appropriate use of personal protective equipment, and cleaning and disinfection to prevent acquisition of CF pathogens from the contaminated healthcare environment. Furthermore, the CF community is encouraged to share best practices, written policies, quality improvement 
initiatives, educational materials, strategies for non-face-toface interactions among individuals with $\mathrm{CF}$, and outcome studies related to IP\&C practices.

The key recommendations in this document that are new for the CF community are as follows:

1. Develop strategies to monitor adherence to IP\&C practices by healthcare personnel and provide them with feedback for improvement.

2. Partner with IP\&C teams to implement the recommendations in this guideline, especially those that are likely to be followed in areas of the facility that are not dedicated only to people with CF.

3. Implement Contact Precautions (ie, wear a gown and gloves) when caring for all people with $\mathrm{CF}$, regardless of respiratory tract culture results, in both ambulatory and inpatient settings.

4. Separate all people with CF from others with CF, regardless of their respiratory tract culture results, at least 6 feet (2 meters) in all settings, to reduce the risk of droplet transmission of CF pathogens.

5. All people with CF and their family members and friends should perform appropriate hand hygiene (with either alcohol-based hand rub or antimicrobial soap and water) when there is the potential for contamination of hands with pathogens. Contamination of hands may occur when entering and exiting a CF clinic, clinic exam room, or hospital room or from respiratory secretions after coughing, performing pulmonary function tests, or performing chest physiotherapy.

6. All people with CF, regardless of respiratory tract culture results, should wear a surgical (also called procedure or isolation) mask when in a healthcare setting to reduce the risk of transmission or acquisition of CF pathogens.

7. Perform pulmonary function tests (PFTs) to reduce transmission from one person with $\mathrm{CF}$ to another person with CF by performing the test in one of the following ways:

- In the exam room at the beginning of the clinic visit, allowing 30 minutes to elapse between CF patients;

- In a negative pressure room (airborne infection isolation room);

- In a PFT laboratory with high-efficiency particulate (HEPA) filters; or

- In a PFT laboratory without HEPA filters, allowing 30 minutes to elapse between individuals with CF.

8. Updated recommendations for care of nebulizers in the hospital.

9. Only 1 person with CF may attend a CF Foundationsponsored indoor event.

\section{I-III. Background Information Supporting the Recommendations}

\section{CF Microbiology and Molecular Typing}

The recommendations for processing CF respiratory tract specimens in the 2003 Infection Control Guideline for $\mathrm{CF}^{1}$ are endorsed in the updated guideline. Several molecular typing strategies are reviewed, with a focus on newer technologies, such as whole-genome sequencing. The importance of international efforts in understanding the molecular epidemiology of CF pathogens is discussed, as is the need to use molecular epidemiology as one tool to monitor the success of IP\&C strategies. An update on the epidemiology of CF pathogens is provided, with an emphasis on gram-negative pathogens, including Burkholderia spp., small colony variant $S$. aureus, and nontuberculous mycobacteria (NTM). This section also emphasizes the importance of surveillance strategies to assess the impact of therapeutic interventions, to identify potential outbreaks, and to monitor the success of IP\&C strategies.

\section{Routes of Transmission of CF Pathogens}

In this section, as in the 2003 Infection Control Guideline for CF, the importance of contact and droplet transmission is emphasized. While the precise routes of transmission are unclear for every acquisition, data support transmission by direct contact with infectious secretions; indirect contact with infectious secretions through contaminated intermediate objects, such as healthcare surfaces, equipment, or the hands of healthcare personnel; and/or infectious droplets. New data are provided demonstrating that droplets can travel as far as 6 feet ( 2 meters), the complexities of droplet transmission are described, and the potential role played by droplet nuclei in transmission of CF pathogens is discussed.

\section{Potential Sources of CF Pathogens}

In this section, as in the 2003 Infection Control Guideline for CF, it is again emphasized that the source of CF pathogens is often unknown and that many individuals with $\mathrm{CF}$ are infected with unique strains. However, molecular epidemiology tools have expanded the evidence that people with CF can share epidemic strains of P. aeruginosa, Burkholderia spp., MRSA, and M. abscessus. Less commonly, strains of other gram-negative pathogens (eg, Stenotrophomonas maltophilia, Achromobacter xylosoxidans, Ralstonia, Cupriavidus, and Pandoraea spp.) may be shared by people with CF. The adverse clinical impact of epidemic strains is highlighted. The sources of and role played by filamentous fungi in CF lung disease (eg, Aspergillus spp.) are also considered.

While transmission of CF pathogens among people with $\mathrm{CF}$ is very well described, transmission of CF pathogens from individuals without $\mathrm{CF}$ to individuals with CF appears to be almost exclusively limited to respiratory viral pathogens. Acquisition of CF pathogens from animals has not been described. In contrast, acquisition from the natural environment (ie, soil, organic matter, and water) is feasible given the ecologic niches of some CF pathogens. The potential for acquisition of CF pathogens from contaminated healthcare environmental sources, including water, surfaces, equipment, air, and products, is also discussed. 
IV. Strategies to Reduce Transmission and Acquisition of CF Pathogens

This section is divided into 9 subsections that describe IP\&C strategies and the rationale for implementing them for all people with $\mathrm{CF}$, including those who have undergone lung or liver transplantation. The subsections include education strategies for healthcare personnel and for people with CF and their families, including audits and feedback for healthcare personnel performance; hand hygiene for healthcare personnel, people with CF, and their families; use of personal protective equipment, including the appropriate use of gowns and gloves by healthcare personnel for all interactions with people with CF; mask use by healthcare personnel as per CDC recommendations; mask use by individuals with CF; cleaning and disinfection of the healthcare environment and equipment, including recommendations for nebulizer care; $\boldsymbol{C F}$ clinic strategies, including recommendations for performing pulmonary function testing; transmission-based precautions for hospitalized people with CF; construction and renovation; and strategies for nonhealthcare settings (eg, camps, indoor and outdoor events, and schools).

Three additional sections have been developed: "Healthcare Personnel with CF" (Section V), which provides the recommendation that people with CF who are interested in healthcare professions should seek advice from their CF care teams about lower-risk options on the basis of their health status; "Psychosocial and Medical Impact of TransmissionBased Isolation Precautions" (Section VI), in which the unintended consequences of transmission precautions in both $\mathrm{CF}$ and non-CF patient populations as well as strategies to mitigate these are described, including developing non-faceto-face methods of communicating among people with CF; and "Challenges to Implementation of IP\&C Recommendations" (Section VII), in which challenges experienced by healthcare personnel and by individuals with CF and their families-as well as strategies to overcome them-are discussed.

\section{Tables, Figures, and Research Agenda}

Tables are provided to supplement the text. These include (1) the population, intervention, comparison, and outcome (PICO) questions the committee developed for the systematic review; (2) a review of the grading systems used; (3) the species in the B. cepacia complex; (4) examples of hand hygiene opportunities for healthcare personnel, people with CF, and their families; (5) indications for use of personal protective equipment by healthcare personnel, people with $\mathrm{CF}$, and their families; (6) strategies to enhance the effectiveness of environmental cleaning in healthcare settings; (7) strategies for CF clinics to minimize risk of transmission of potential pathogens; (8) strategies to minimize the adverse psychosocial impact of isolation precautions; (9) knowledge, attitudes, and practice barriers related to implementing IP\&C in CF; and (10) strategies to enhance implementation of IP\&C in CF. Two figures are also presented: the age-specific prevalence of
CF pathogens in 2012 in the United States, and the changing prevalence of CF pathogens in the United States from 1988 to 2012 .

A research agenda is proposed to address some of the unresolved IP\&C issues for the CF community, including, for example (1) the role played by small colony variant $S$. aureus; (2) the frequency of shared strains of CF pathogens, including P. aeruginosa and NTM in the United States; (3) the routes of transmission of M. abscessus; (4) the role played by specific niches for CF pathogens in the natural environment; (5) continued efforts to define best IP\&C practices for CF; (6) continued efforts to assess and overcome challenges to implementation of IP\&C; and (7) additional research into the unique needs of healthcare personnel with $\mathrm{CF}$.

\section{Summary}

In summary, epidemiologic studies have shown that pathogens, other than Burkholderia spp., can be transmitted among individuals with CF, resulting in adverse clinical outcomes, including increased morbidity and mortality. The updated guideline is a response to new knowledge and new challenges in both IP\&C and CF. The primary objective of the guideline is to provide recommendations to reduce the risk of transmission and acquisition of CF pathogens by individuals with CF and to provide a more comprehensive understanding of effective strategies to optimize safety for this unique population.

\section{N T RODUCTION}

In 2003, the Cystic Fibrosis (CF) Foundation published recommendations for infection prevention and control (IP\&C) in an effort to reduce the risk of acquisition and transmission of pathogens among people with CF. ${ }^{1}$ However, both IP\&C and $\mathrm{CF}$ are dynamic disciplines, and during the past decade new knowledge and new challenges necessitated the development of updated IP\&C strategies for this unique population.

1. IP\&C experiences in the general population can provide insight into strategies for people with CF. Numerous evidence-based guidelines for IP\&C and clinical practice guidelines have been published since 2003 by the Centers for Disease Control and Prevention (CDC)/Healthcare Infection Control Practices Advisory Committee (HICPAC), the World Health Organization (WHO), and professional societies, including the Infectious Diseases Society of America (IDSA) and the Society for Healthcare Epidemiology of America (SHEA). These guidelines contain relevant recommendations for people with CF (see Table 1 for the most relevant guidelines). ${ }^{2-13}$

In addition, each year the CDC/Advisory Committee on Immunization Practices (ACIP) updates the recommendations for immunizations in children and adults and the recommendations for the prevention of influenza for the upcoming season; these recommendations are published in Morbidity and Mortality Weekly Report (MMWR). ${ }^{14}$ Relevant 
TABLE 1. Infection Prevention and Control Guidelines Published since 2003

\begin{tabular}{lc}
\hline Guideline & Organization \\
\hline Guidelines for Environmental Infection Control in Health-Care Facilities, 2003 & CDC/HICPAC \\
Guidelines for Preventing Health-Care-Associated Pneumonia, 2003 & CDC/HICPAC \\
Guidelines for Preventing the Transmission of Mycobacterium tuberculosis in Health-Care & CDC/NCHHSTP \\
$\quad$ Settings, 2005 & CDC/HICPAC \\
Management of Multidrug-Resistant Organisms in Health Care Settings, 2006 & CDC/HICPAC \\
Guideline for Isolation Precautions: Preventing Transmission of Infectious Agents in Health & CDC/HICPAC \\
$\quad$ Care Settings, 2007 & 5 \\
Disinfection and Sterilization in Health-Care Facilities, 2008 & IDSA/SHEA \\
Strategies to Prevent Transmission of Methicillin-Resistant Staphylococcus aureus in Acute Care & WHO \\
$\quad$ Hospitals, 2008 & CDC/HICPAC \\
Guidelines on Hand Hygiene in Healthcare, 2009 & 7 \\
Infection Prevention for Outpatient Settings: Minimum Expectations for Safe Care, 2011 & CDC/ACIP \\
Immunization of Health-Care Personnel: Recommendations of the Advisory Committee on & 8 \\
$\quad$ Immunization Practices & SHEA \\
Infection Prevention and Control in Residential Facilities for Pediatric Patients and Their & IDSA \\
$\quad$ Families, 2013 & 11 \\
Clinical Practice Guideline for Vaccination of the Immunocompromised Host, 2013 & 12 \\
\hline
\end{tabular}

note. ACIP, Advisory Committee on Immunization Practices; CDC, Centers for Disease Control and Prevention; HICPAC, Healthcare Infection Control Practices Advisory Committee; HIV, human immunodeficiency virus; IDSA, Infectious Diseases Society of America; NCHHSTP, National Center for HIV/AIDS, Viral Hepatitis, STD, and TB Prevention; SHEA, Society for Healthcare Epidemiology of America; STD, sexually transmitted diseases; TB, tuberculosis; WHO, World Health Organization.

recommendations from both IP\&C guidelines and ACIP recommendations have been integrated into this updated IP\&C guideline for people with CF. Furthermore, during the past decade new evidence has led to a renewed emphasis on source containment of potential pathogens, the role played by the contaminated healthcare environment in transmitting infectious agents, and an increased understanding of the importance of implementation science, monitoring adherence, and feedback principles to enhance the effectiveness of IP\&C practices as detailed throughout this document.

2. Experience with emerging pathogens can inform IPঊC strategies for $\mathrm{CF}$. The severe acute respiratory syndrome coronavirus (SARS-CoV) and the 2009 influenza A H1N1 pandemic expanded our understanding of droplet transmission of infectious agents. ${ }^{6,14,15}$ While the incidence of healthcareand community-associated infections caused by methicillinresistant Staphylococcus aureus (MRSA) has been decreasing in adults in the United States in recent years ${ }^{16}$ a similar trend has not been observed among children. ${ }^{17}$ Additionally, multidrug-resistant gram-negative pathogens continue to emerge and are increasing in healthcare settings ${ }^{18}$ which has heightened the awareness of healthcare personnel and the public of the importance of preventing patient-to-patient transmission of multidrug-resistant organisms (MDROs)., ${ }^{5,19,20}$

3. Changes in the demographic characteristics of the CF population and in the epidemiology of CF pathogens during the past decade have implications for IPঊC. The median predicted life expectancy of people with CF has increased to 41 years of age. ${ }^{21}$ In the United States and in many other countries, the number of adults with CF is comparable to or has surpassed the number of children with CF. Newborn screening for $\mathrm{CF}$ is now mandated in all 50 states in the United States. Newer CF pathogens are increasing in prevalence and are associated with increased rates of morbidity and mortality among individuals with CF. Such pathogens include MRSA, ${ }^{22,23}$ Mycobacteria abscessus, ${ }^{24}$ Burkholderia dolosa ${ }^{25}$ new species in the Burkholderia cepacia complex, ${ }^{26}$ epidemic clones of Pseudomonas aeruginosa (eg, the Liverpool epidemic strain [LES]), ${ }^{27-29}$ and others. ${ }^{30}$ Thanks to the increasing use of molecular typing, strains that are shared among people with CF continue to be identified, suggesting that new strategies beyond those recommended in the 2003 Infection Control Guideline for $\mathrm{CF}$ are needed to improve the implementation of IP\&C practices and to reduce the transmission of CF pathogens.

While extraordinary advances have been made in the treatment of CF, including the use of potentiators and correctors aimed at correcting the abnormal CF transmembrane conductance regulator (CFTR), ${ }^{31}$ the CF and the IP\&C communities must continue to prioritize minimizing the risk of acquisition and transmission of CF pathogens. To update the 2003 Infection Control Guideline for CF, the CF Foundation assembled an interdisciplinary committee to (1) review existing literature and present new knowledge that served as the basis for the updated IP\&C practice recommendations; (2) assess the relevance of existing guidelines and integrate appropriate recommendations, including those from the 2003 Infection Control Guideline for CF; (3) craft updated recommendations; and (4) address strategies to improve implementation of IP\&C practices, including education and overcoming challenges to implementation.

The process the committee undertook to complete its tasks, 
including the systematic review, is described below. The groups of updated IP\&C recommendations are as follows: (I) core recommendations for all people with CF (including following lung or liver transplantation) in all settings; (II) recommendations for microbiology and molecular epidemiology; (III) recommendations for CF clinics and other ambulatory care settings; (IV) recommendations for inpatient settings; (V) recommendations for nonhealthcare settings; (VI) recommendations for healthcare personnel with CF; and (VII) recommendations for the psychosocial and medical impact of IP\&C. To facilitate use of the guideline, the relevant sections of "Background Information Supporting the Recommendations" (Sections I-III) and strategies to reduce transmission and acquisition of pathogens (Sections IV-VII) are provided for each recommendation as supporting rationale.

This guideline is intended for use by all healthcare personnel involved with the care of people with CF and the IP\&C teams that support CF care centers in the United States. The recommendations for healthcare settings are intended to be implemented in CF clinics and other ambulatory care areas, in inpatient settings, in diagnostic and therapeutic areas, and during all clinical research activities. The recommendations for nonhealthcare settings presented in this updated guideline are not intended to be enforced by healthcare personnel but instead represent efforts to respond to questions and concerns voiced by people with CF and their caregivers and to provide education about the potential risks associated with various activities or exposures. People with CF and their families and friends will then be better prepared to make informed choices in their personal lives.

\section{METHODS FOR DOCUMENT DEVELOPMENT}

\section{Committee Structure}

In March 2011, the CF Foundation requested volunteers to participate in developing an update of the 2003 Infection Control Guideline for CF. The 21-member interdisciplinary committee consisted of 4 infectious disease specialists (all of whom had expertise in IP\&C and CF microbiology), 4 pulmonologists, 4 nurses, 1 respiratory therapist, 1 infection preventionist, 3 parents of children with CF, 1 adult with CF, 1 social worker, and 2 CF Foundation staff members (a pulmonologist and a nurse).

In November 2011, the committee assembled and developed the scope of the guidelines by identifying clinical questions to be addressed, using the population, intervention, comparison, and outcome (PICO) format. ${ }^{32}$ To evaluate the published evidence for answers to these questions, the CF Foundation commissioned an evidence review from a Johns Hopkins University team under the leadership of an epidemiologist (K.A.R.) with experience in conducting systematic reviews, including those assessing interventions used in the CF population. The PICO questions used to guide the evidence search are presented in Table 2.

\section{Systematic Review Process}

For the systematic review, searches of PubMed, Embase, and the Cochrane Central Register of Controlled Studies were conducted by the Johns Hopkins University research team in June 2012. Searches of reference lists for all eligible articles and Cochrane reviews were also completed. Committee members provided additional potentially eligible studies. Studies performed in the CF population were sought preferentially, but studies conducted in other populations considered relevant were also reviewed. Two independent reviewers screened search results for eligible studies. Details about eligible studies were abstracted and a report, including evidence tables and qualitative synthesis, was submitted to the CF Foundation and disseminated to the committee.

Additionally, the Johns Hopkins University research team identified relevant guidelines and Cochrane reviews through searches (completed in August 2012) of the National Guidelines Clearinghouse, United Kingdom CF Trust website, CF Foundation guidelines database, the Cochrane Library, and lists provided by the committee chairs. Details from these sources, including recommendation statements, were abstracted and provided to the committee.

\section{Results of the Systematic Review}

The search identified 16 eligible articles reporting 15 unique studies. These included 4 before-and-after studies, 4 crosssectional studies, and 7 nonconcurrent cohort studies, but these studies provided insufficient evidence to use the US Preventive Services Task Force grading system that has been used for other recent CF practice guidelines. ${ }^{33,34}$ The systematic review team also abstracted 2,403 recommendation statements from 47 relevant IP\&C guidelines. Evidence tables can be obtained from the CF Foundation on request (resources@cff.org).

\section{Process for InClusion of Recommendations}

Each recommendation from the 2003 Infection Control Guideline for CF was reviewed for continued relevance and modified if clarification was needed or if new data were available. The grade of evidence from the 2003 Infection Control Guideline for CF was retained. Relevant recommendations from other CF practice guidelines were included verbatim. Relevant recommendations from other guidelines developed for non-CF populations by other expert professional organizations, including CDC/HICPAC, SHEA, IDSA, WHO, APIC, and ACIP were also included, and their grade of evidence was retained without a vote by the committee (Table 3). ${ }^{1,9}$ New recommendations that had not been published previously were developed by the committee. Inclusion of the recommendations was determined by anonymous voting. At least $80 \%$ approval by the committee members (ie, consensus) was set as the threshold for acceptance of new recommendations, relevant recommendations from the 2003 Infection 
TA B LE 2. Population, Intervention, Comparison, and Outcome (PICO) Clinical Questions Developed for the Guideline for Infection Prevention and Control in Cystic Fibrosis (CF): 2013 Update

\section{Microbiology}

1. Does identification of small colony variant Staphylococcus aureus versus not performing identification of small colony variant $S$. aureus affect clinical outcomes of people with $\mathrm{CF}$ experiencing exacerbation?

Transmission: Personal Protective Equipment

2a. What is the evidence for benefit or harm of people with CF wearing masks in the healthcare setting versus not wearing masks?

2b. What is the evidence for benefit or harm of healthcare providers wearing masks versus not wearing masks when caring for people with $\mathrm{CF}$ ?

3a. What is the evidence for benefit or harm of people with wearing gowns versus not wearing gowns in healthcare settings?

3b. What is the evidence for benefit or harm of healthcare providers wearing gowns versus not wearing gowns when caring for people with $\mathrm{CF}$ ?

4a. What is the evidence for benefit or harm of people with CF wearing gloves versus not wearing gloves in healthcare settings?

$4 \mathrm{~b}$. What is the evidence for benefit or harm of healthcare providers wearing gloves in healthcare setting versus not wearing gloves among people with CF?

Transmission: Distance for Droplets

5. What is the evidence that more than 3 feet distance between people with CF versus 3 feet or less is required to prevent droplet transmission?

Transmission: Methicillin-Resistant S. aureus (MRSA)

6a. What is the evidence that separation versus nonseparation reduces MRSA transmission from people without CF who have skin and soft-tissue infection (SSTI) to prevent respiratory tract infection in people with CF?

6b. What is the evidence that separation versus nonseparation of people with CF with MRSA respiratory tract infections reduces MRSA transmission to others with CF?

6c. What is the evidence that separation versus nonseparation of people with CF with MRSA respiratory tract infections reduces MRSA transmission and prevents SSTI in people without CF?

Transmission: Nonhealthcare Settings

7. What is the evidence that restriction versus nonrestriction reduces transmission of CF pathogens in indoor and/or outdoor nonhealthcare settings where more than 1 person with CF is present (eg, fund-raising events, cystic fibrosis chapter offices, pharmaceutical company venues, Great Strides)?

8a. What is the evidence among people with $\mathrm{CF}$ that restriction versus nonrestriction reduces transmission of CF pathogens from leisure activities involving soil and plants (eg, gardening and lawn care)?

8b. What is the evidence that among people with CF restriction versus nonrestriction reduces transmission of CF pathogens from leisure activities involving aquatic settings, hot tubs, swimming pools, and natural bodies of water?

Transmission: Animals

9a. What is the evidence that among people with CF restriction versus nonrestriction reduces transmission of CF pathogens from pet therapy?

9b. What is the evidence that among people with CF restriction versus nonrestriction reduces transmission of CF pathogens from personal pets or farm animals?

Transmission: Healthcare Personnel with CF

10. What is the evidence that a healthcare provider with CF should be restricted versus not restricted from working with people with CF to prevent transmission of CF pathogens from a healthcare provider with CF to his or her patients and vice versa?

Transmission: Scheduling CF Clinic Visits

11. What is the evidence that scheduling CF clinic visits on the basis of pathogen status (separate clinic times) versus not scheduling CF clinic visits on the basis of pathogen status (no separate clinic times) reduces transmission of CF pathogens?

Cleaning Respiratory Equipment

12a. What is the evidence for cleaning and disinfecting respiratory equipment of people with CF after each use versus some other frequency for cleaning to prevent contamination and transmission of CF pathogens in the hospital?

12b. What is the evidence for cleaning and disinfecting respiratory equipment of people with CF after each use versus some other frequency for cleaning to prevent contamination and transmission of CF pathogens in the home?

12c. What is the evidence for cleaning and disinfecting respiratory equipment of people with CF with one method versus another method for cleaning to prevent contamination and transmission of CF pathogens in the hospital?

$12 \mathrm{~d}$. What is the evidence for cleaning and disinfecting respiratory equipment of people with $\mathrm{CF}$ with one method versus another method for cleaning to prevent contamination and transmission of CF pathogens in the home?

Control Guideline for CF, or relevant recommendations from other guidelines developed for non-CF populations.

The CDC/HICPAC guidelines and the WHO hand hygiene guideline cited in this document used a unique HICPAC grad- ing system that was used for HICPAC guidelines published before 2009. The Grading of Recommendations, Assessment, Development, and Evaluation (GRADE) system $^{35}$ was adopted for use by CDC/HICPAC/ACIP in $2009^{36,37}$ and is 
тавLE 3. Grading Systems Used in the Updated Infection Prevention and Control (IP\&C) Guidelines for Cystic Fibrosis (CF), 2013

\begin{tabular}{|c|c|c|}
\hline Source of recommendation & Grading strategy & Comments \\
\hline \multirow{2}{*}{$\begin{array}{l}2003 \text { Infection Control Guideline } \\
\text { for } \mathrm{CF}^{1} \\
\text { and }\end{array}$} & Category IA & $\begin{array}{l}\text { Strongly recommended for implementation and strongly supported by well- } \\
\text { designed experimental, clinical, or epidemiologic studies }\end{array}$ \\
\hline & & \\
\hline \multirow{4}{*}{$\begin{array}{l}\text { CDC HICPAC guidelines }{ }^{2,3,5-7,10} \\
\text { and } \\
\text { WHO Guidelines on Hand Hy- } \\
\text { giene in Healthcare, } 2009^{9}\end{array}$} & Category IB & $\begin{array}{l}\text { Strongly recommended for implementation and supported by some experimen } \\
\text { tal, clinical, or epidemiologic studies and a strong theoretical rationale }\end{array}$ \\
\hline & Category IC & $\begin{array}{l}\text { Required for implementation, as mandated by federal and/or state regulation } \\
\text { or standard }\end{array}$ \\
\hline & Category II & $\begin{array}{l}\text { Suggested for implementation and supported by suggestive clinical or epidemi- } \\
\text { ologic studies or a theoretical rationale }\end{array}$ \\
\hline & No recommendation & $\begin{array}{l}\text { Unresolved issue; }{ }^{\mathrm{a}} \text { practices for which insufficient evidence or no consensus re- } \\
\text { garding efficacy exist }\end{array}$ \\
\hline Systematic review & Not applicable & $\begin{array}{l}\text { Limited evidence was found by the systematic review conducted for the } 2013 \\
\text { IP\&C guideline; thus, the grading of evidence was not used }\end{array}$ \\
\hline \multirow{2}{*}{$\begin{array}{l}2013 \text { Updated IP\&C Guideline } \\
\text { for CF }\end{array}$} & $\%$ agreement & $\geq 80 \%$ agreed with statement by anonymous voting \\
\hline & Certainty: low & $\begin{array}{l}<80 \% \text { agreed with statement by anonymous voting and thus insufficient evi- } \\
\text { dence exists to recommend for or against }\end{array}$ \\
\hline
\end{tabular}

note. CDC, Centers for Disease Control and Prevention; HICPAC, Healthcare Infection Control Practices Advisory Committee; WHO, World Health Organization.

a The unresolved issue category was not included in the 2009 WHO hand hygiene guidelines.

used by more than 70 organizations worldwide. However, the GRADE system can result in IP\&C guidelines that are more likely to include expert consensus compared with guidelines developed for specific treatment regimens that have randomized clinical trials as their evidence base. Limitations of the GRADE system that may impact developing IP\&C guidelines include (1) questions for which little or no evidence is available on which to base a recommendation, (2) little or no requirement for evidence given the high probability of a recommendation's success, and (3) difficulty assessing the strength of evidence from studies performed in varying populations with varying study designs. ${ }^{37,38}$ The evidence base for this updated guideline had similar limitations; thus, the recommendations in this guideline are often based on expert consensus.

\section{External Review}

In May 2013, the CF Foundation made a draft of the updated guideline available for comment to the CF community, including the teams at CF care centers and people with CF. Infection preventionists and healthcare epidemiologists were also notified of the availability of the document for comment. All comments were considered by the committee, and the recommendations and background information were revised as appropriate. This guideline was reviewed and endorsed by SHEA and by APIC.
UPDATED RECOMMENDATIONS FOR I P \& C IN C F

\section{Core Recommendations}

The CF Foundation recommends implementation of the following core IP\&C recommendations to minimize the risk of transmission and acquisition of pathogens among all people with CF, including following lung or liver transplantation, in all settings.

\section{Education/Adherence Monitoring for Healthcare Personnel, People with CF, and Families}

1. The CF Foundation recommends that all healthcare personnel caring for people with CF (eg, the CF care team, inpatient staff, environmental services staff, research staff, and staff in diagnostic and therapeutic areas, including pulmonary function test [PFT] laboratories, radiology, phlebotomy, operating room, and physical therapy) receive education regarding IP\&C for CF, using principles of adult learning. Education should be repeated at intervals each center deems appropriate.

Source of supporting evidence: 2003 CF IP\&C guideline, Category II; 2006 MDRO guideline, Category IB; 2007 transmission guideline, Category $I B$

2013 CF IP\&C guideline consensus: $100 \%$

Sections in the text: III.D.2; IV.B 
2. The CF Foundation recommends that the CF care team develop strategies to monitor adherence to IP\&C practices by healthcare personnel and provide feedback. Feedback to the CF care team includes immediate feedback to an individual when a lapse in practice is observed and feedback to the entire CF care team of trends of overall adherence rates at regular intervals (eg, quarterly) on the basis of consistency of practices.

\section{Source of supporting evidence: 2003 CF IP\&C guideline, Category IB; 2006 MDRO guideline, Category IB; 2007 transmission guideline, Category IB \\ 2013 CF IP\&C guideline consensus: $100 \%$ \\ Sections in the text: IV.B; IV.E.1}

3. The CF Foundation recommends that all people with $\mathrm{CF}$ and their families receive education regarding IP\&C for $\mathrm{CF}$, using age appropriate tools and reading/language level appropriate to the target audience. Involve people with $\mathrm{CF}$ and their families in the development of educational programs and implementation of recommended practices. Education should be repeated at intervals each center deems appropriate.

Source of supporting evidence: 2003 CF IP\&C guideline, Category II

2013 CF IP\&C guideline consensus: $100 \%$

Sections in the text: IV.B

\section{Partnering with Institutional IPঊC Teams}

4. The CF Foundation recommends that CF care teams collaborate with their institutional IP\&C teams to implement the recommendations in this guideline.

Source of supporting evidence: 2006 MDRO guideline, Category IB

2013 CF IP\&C guideline consensus: $100 \%$

Sections in the text: I.D; IV.B; IV.E.1, 2; IV.F

5. The CF Foundation recommends that CF care teams collaborate with their institutional IP\&C teams to develop protocols, checklists, and audits to standardize implementation of practices for the following:

a. Single-patient-use, disposable items

b. Cleaning and disinfecting multiuse items (eg, patient care equipment, oximeters, iPads and similar tablets, and computers)

c. Cleaning and disinfecting surfaces in the healthcare environment (eg, CF clinics, PFT rooms, hospital rooms, and sinks and showers)

Source of supporting evidence: 2008 disinfection and sterilization guideline, Category II; http://www.cdc.gov /HAI/toolkits/Evaluating-Environmental-Cleaning.html 2013 CF IP\&C guideline consensus: $\mathbf{1 0 0} \%$ Sections in the text: III.D.2; IV.E.2; IV.F.6, 7

6. The CF Foundation recommends ensuring that dust containment during renovation and construction and water- leak remediation policies and practices are followed according to institutional and national guidelines in all ambulatory care areas and inpatient settings where people with CF receive care.

Source of supporting evidence: 2003 CF IP\&C guideline, Category IB/IC; 2003 CDC environmental guideline, Category $I B / I C$

2013 IP\&C guideline consensus: 100\%

Sections in the text: III.D.3; IV.H

7. The CF Foundation recommends that healthcare personnel assume that all people with CF could have pathogens in respiratory tract secretions that are transmissible to other people with $\mathrm{CF}$.

Source of supporting evidence: 2003 CF IP\&C guideline, Category IA

2013 CF IP\&C guideline consensus: $100 \%$

Sections in the text: IV.F.1; IV.G

\section{Practices for Healthcare Personnel}

8. The CF Foundation recommends that all healthcare facilities caring for people with CF ensure ready availability of alcohol-based hand rub or antimicrobial soap and water in all patient rooms, PFT rooms, and waiting areas.

Source of supporting evidence: 2003 CF IP\&C guideline, Category IA; 2009 WHO and 2002 hand hygiene guidelines, Category IA

2013 CF IP\&C guideline consensus: $100 \%$

Sections in the text: IV.B; IV.F.3

9. The CF Foundation recommends that healthcare personnel perform hand hygiene (either using alcohol-based hand rub or washing hands with antimicrobial soap and water), as per CDC and WHO guidelines, in the following clinical situations:

a. Before entering the room and when leaving the room of any patient

b. Before and after direct contact with any patient

c. Before putting gloves on and after removing gloves, for both sterile and nonsterile procedures

d. After contact with patient's skin, mucous membranes, respiratory secretions, or other body fluids

e. After contact with inanimate objects (including medical equipment) in the vicinity of the patient that may be potentially contaminated with respiratory secretions

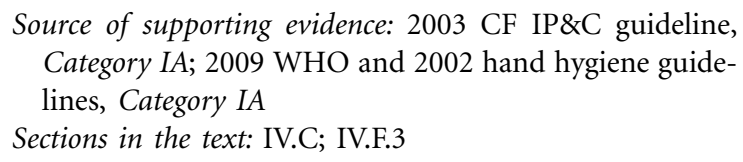

10. The CF Foundation recommends that healthcare personnel should not wear artificial fingernails or nail extenders when having direct contact with people with CF. 
Source of supporting evidence: 2002 HICPAC hand hygiene, Category IA for high-risk patients; 2009 WHO hand hygiene, Category IA for all patients

Sections in the text: IV.C

11. The CF Foundation recommends that healthcare personnel should disinfect their stethoscopes before and after use on each patient in accordance with institutional IP\&C policies. Stethoscopes that remain in the patient's room and are dedicated for use only for that patient do not need to be disinfected before and after use.

Source of supporting evidence: 2006 MDRO guideline, Category IB

2013 CF IP\&C guideline consensus: $100 \%$

Sections in the text: III.D.2; IV.E.2; IV.F.7; IV.G

12. The CF Foundation recommends that healthcare personnel caring for people with CF should not be routinely screened for MRSA colonization unless they are epidemiologically linked to a cluster of MRSA infections in accordance with institutional IP\&C policies and national guidelines.

Source of supporting evidence: 2006 MDRO guideline, Category $I B$

Sections in the text: III.B.2

\section{Isolation Precautions}

13. The CF Foundation recommends that all healthcare personnel implement Contact Precautions (ie, wear a gown and gloves) when caring for all people with CF regardless of respiratory tract culture results, in ambulatory and inpatient settings.

Source of supporting evidence: 2007 transmission guideline, Category $I B / I C$

2013 CF IP\&C guideline consensus: $100 \%$

Sections in the text: II.A; IV.D.1; IV.G

14. The CF Foundation does not recommend that healthcare personnel wear a mask routinely when caring for people with CF. However, the CF Foundation recommends mask use per CDC guidelines, as follows:

a. Surgical (procedure, isolation) masks are worn by healthcare personnel caring for any patient under Droplet Precautions with suspected or confirmed pathogens that are transmitted by the droplet route (eg, adenovirus, rhinovirus, influenza virus, or Mycoplasma pneumoniae).

b. Masks and eye protection should be worn by healthcare personnel if splashes or sprays of respiratory tract secretions are anticipated as per Standard Precautions.

c. N-95 respirators (masks) or powered air-purifying respirators (PAPRs) are worn by healthcare personnel caring for any patient under Airborne Precautions (in an airborne infection isolation room [AIIR]) for suspected or confirmed infection with Mycobacterium tuberculosis.
Source of supporting evidence: 2003 CF IP\&C guideline, Category IA; 2007 transmission guideline, Category IB; 2007 transmission guideline, Category IB; 2005 tuberculosis $(\mathrm{TB})$ transmission guideline

Sections in the text: II.C; IV.D.2; IV.G

15. The CF Foundation recommends placing people with CF who are acid-fast bacilli (AFB) smear positive for the first time under Airborne Precautions (AIIR requirements: negative-pressure single room, more than 12 air exchanges per hour, air exhausted to the outside) in ambulatory and inpatient settings until $M$. tuberculosis infection has been excluded. Alternatively, in geographic locations with a very low incidence of TB, a risk assessment that includes the likelihood of exposure to individuals with TB (eg, travel or visitors from high-prevalence areas) may be used to guide the use of AIIRs. Consult with institutional IP\&C staff and/or infectious disease physicians.

Source of supporting evidence: 2003 CF IP\&C guideline, Category IA; 2005 M. tuberculosis transmission guideline; 2007 transmission guideline, Category IA/IC

2013 CF IP\&C guideline consensus: $100 \%$

Sections in the text: IV.D.2; IV.G

16. The CF Foundation concludes that there is insufficient evidence at the time of publication of this document for or against placing people with CF who are infected with NTM under Airborne Precautions.

2013 CF IP\&C guideline, certainty: low

Sections in the text: III.A.5; III.D.2; IV.G

\section{Practices by People with CF and Family Members/Friends}

17. The CF Foundation recommends that all people with $\mathrm{CF}$, regardless of their respiratory tract culture results, be separated by at least 6 feet ( 2 meters) from other people with CF in all settings, to reduce the risk of droplet transmission of CF pathogens. This does not apply to members of the same household.

2013 CF IP\&C guideline consensus: $100 \%$

Sections in the text: II.A; II.B; II.C; III.D.2; IV.F.1, 2

18. The CF Foundation recommends that all people with $\mathrm{CF}$ and their family members/friends perform hand hygiene (with either alcohol-based hand rub or antimicrobial soap and water) when there is potential for contamination of hands with pathogens, such as the following:

a. Entering and exiting CF clinics, clinic exam rooms, or hospital rooms

b. Hands become contaminated with respiratory secretions (eg, after coughing or performing PFTs or chest physiotherapy)

Source of supporting evidence: 2003 CF IP\&C guideline, Category IA

2013 CF IP\&C guideline consensus: 100\%

Sections in the text: II.A; IV.C; IV.F.3 
19. The CF Foundation does not recommend that people with CF wear gowns or gloves in CF clinics, in other ambulatory healthcare settings, or while hospitalized.

2013 CF IP\&C guideline consensus: $100 \%$

Sections in the text: IV.C; IV.D.1

20. The CF Foundation recommends that people with CF be instructed to follow Respiratory Hygiene practices to contain their secretions when coughing or sneezing (ie, cough into a tissue, immediately discard soiled tissue into a trash receptacle, and perform hand hygiene after disposing of soiled tissues). A covered trash receptacle with a foot pedal is preferred.

Source of supporting evidence: 2003 CF IP\&C guideline,

Category II; 2007 transmission guideline, Category IB

2013 CF IP\&C guideline consensus: $100 \%$

Sections in the text: IV.A; IV.D.2; IV.F.4

21. The CF Foundation recommends that all people with CF wear a surgical (procedure, isolation) mask when in a healthcare facility to reduce the risk of transmission or acquisition of CF pathogens. Masks should be worn throughout the facility, including in restrooms. Masks should not be worn during pulmonary function testing, in the clinic exam room, or in the patient's hospital room. If the optimal size mask is not available (eg, for small infants), use the smallest mask available. If a mask is not tolerated by an individual with CF who is having respiratory distress, encourage that person to follow Respiratory Hygiene practices. Masks should be changed when wet.

Source of supporting evidence: 2007 transmission guideline, Category $I B$

2013 CF IP\&C guideline consensus: $100 \%$

Sections in the text: II; IV.F.4

22. The CF Foundation recommends that all people with CF who do not live in the same household avoid activities and risk factors that are associated with transmission of CF pathogens in nonhealthcare and healthcare settings, including the following:

a. Social contact between people with CF

b. Physical contact between people with CF (eg, handshakes, kissing, and intimate contact)

c. Car rides with another person with $\mathrm{CF}$

d. Sharing hotel rooms with another person with $\mathrm{CF}$

e. Fitness class with another person with $\mathrm{CF}$

Activities that all people with CF, including those who live in same household, should avoid include the following:

a. Sharing personal items (eg, toothbrush and drinking utensils) with another person with $\mathrm{CF}$

b. Sharing respiratory therapy equipment

Source of supporting evidence: 2003 CF IP\&C guideline, Category IA; 2013 residential facility guideline

2013 CF IP\&C guideline consensus: 100\%

Sections in the text: III.A.1; IV.I
23. The CF Foundation recommends that

a. Tap water or well water that meets local public health standards, distilled water, or bottled water may be used by people with $\mathrm{CF}$

i. For drinking

ii. For bathing

iii. For cleaning nebulizers and other respiratory equipment (eg, airway clearance devices, spacers, and neti pots) if followed by disinfection

$i v$. For the water needed for heat disinfection (eg, boiling, microwaving, and steam sterilizing)

b. Only sterile water be used for nasal rinses (eg, neti pots), filling of humidifier reservoirs, and as a final rinse of respiratory equipment (eg, after cold disinfection)

2013 IP\&C guideline consensus: $100 \%$

Sections in the text: III.D.1; III.D.2; IV.E.3; IV.E.4

\section{Immunizations/Influenza Chemoprophylaxis}

24. The CF Foundation recommends that, as per CDC/ ACIP recommendations, all healthcare personnel (unless there is a medical contraindication to immunization) should be immunized or have evidence of immunity to mumps, measles, rubella, varicella, pertussis (Tdap), and hepatitis B and receive an annual influenza immunization.

Source of supporting evidence: MMWR 2013;62(RR-07):143; http://www.cdc.gov/vaccines/schedules/index.html Sections in the text: IV.I.5

25. The CF Foundation recommends that, as per CDC/ ACIP recommendations, all people with CF and their family members/close contacts receive recommended vaccines at the recommended schedule, age, dose, and route of administration unless there is a medical contraindication.

Source of supporting evidence: MMWR 2013;62(RR-07):143; http://www.cdc.gov/vaccines/schedules/index.html

Sections in the text: IV.I.5

26. The CF Foundation recommends use of antiviral chemoprophylaxis or treatment (eg, oseltamivir) for prevention or treatment of influenza according to ACIP recommendations.

Source of supporting evidence: 2003 CF IP\&C guideline, Category $1 \mathrm{~A} ; \mathrm{http}: / /$ www.cdc.gov/flu/professionals/antivirals /summary-clinicians.htm (2012-2013 season)

2013 CF IP\&C guideline consensus: $100 \%$

Sections in the text: IV.I.5

\section{Research Settings}

27. The CF Foundation recommends that for all research activities, people with CF, their family members/friends, and healthcare personnel follow relevant IP\&C recommendations for that healthcare setting.

2013 CF IP\&C guideline consensus: $100 \%$

Sections in the text: III.A 
II. Recommendations FOR Microbiology AND Molecular Epidemiology

In addition to the microbiology recommendations for processing CF respiratory specimens described in the 2003 Infection Control Guideline for CF, the CF Foundation recommends implementation of the following recommendations:

\section{Review Center-Specific Microbiology Data}

28. The CF Foundation recommends that all CF centers obtain and review center-specific quarterly surveillance reports (eg, data from the local clinical microbiology laboratory or the CF Foundation Patient Registry) of the incidence and prevalence of respiratory tract pathogens at their centers. This review should be conducted in collaboration with institutional IP\&C teams and microbiology laboratory directors.

Source of supporting evidence: 2003 CF IP\&C guideline, Category IB; 2006 MDRO guideline, Category IB

2013 CF IP\&C guideline consensus: $100 \%$

Sections in the text: I.D; IV.F.1

\section{Molecular Typing}

29. The CF Foundation recommends that CF isolates of Burkholderia spp. be sent to the laboratory at the University of Michigan (US) for confirmation of identification, speciation, and molecular typing, as follows:

a. All initial isolates from every patient

b. At least 1 isolate per patient per year

c. Any isolates suspected of being associated with transmission or an outbreak

d. Any other nonfermenting gram-negative organism for which species identification remains equivocal after routine analysis should be sent for confirmation of identification

CF Foundation Burkholderia cepacia Research Laboratory and Repository

University of Michigan

8323 MSRB III, SPC 5646

1150 West Medical Center Drive

Ann Arbor, MI 48109-0646

Tel: 734-936-9767; fax: 734-764-4279; e-mail: jlipuma @umich.edu

Source of supporting evidence: 2003 CF IP\&C guideline, Category IB

2013 CF IP\&C guideline consensus: $100 \%$

Sections in the text: I.B; III.A.1

30. The CF Foundation recommends that molecular typing of B. cepacia complex isolates and other microorganisms (eg, $P$. aeruginosa and NTM) be performed when epidemiologically indicated (eg, suspected patient-to-patient transmission).

Source of supporting evidence: 2003 CF IP\&C guideline, Category IA; 2006 MDRO guideline, Category IB

2013 CF IP\&C guideline consensus: $100 \%$

Sections in the text: I.B; I.D; III.A
31. The CF Foundation recommends that molecular typing be performed using an appropriate genotyping method (eg, pulsed-field gel electrophoresis, random-amplified polymorphic DNA polymerase chain reaction [PCR], repetitive sequence-based PCR, or multilocus sequence typing).

Source of supporting evidence: 2003 CF IP\&C guideline, Category IA; 2006 MDRO guideline, Category IB

2013 CF IP\&C guideline consensus: 100\%

Sections in the text: I.B; III.A.2; III.A.4; III.A.5

\section{Surveillance}

32. The CF Foundation and European CF Society (ECFS) recommend that screening cultures for NTM should be performed annually in individuals with a stable clinical course. Culture and smears for AFB from sputum should be used for NTM screening.

In the absence of clinical features suggestive of NTM pulmonary disease, individuals who are not capable of spontaneously producing sputum do not require screening cultures for NTM. The CF Foundation and ECFS recommend against the use of oropharyngeal swabs for NTM screening.

Source of supporting evidence: 2013 NTM in CF guideline

2013 IP\&C guideline consensus: 100\%

Sections in the text: I.C.5; III.A.5

33. The CF Foundation concludes that there is insufficient evidence at the time of publication of this document to recommend criteria by which to consider a person with CF who previously had Burkholderia species isolated from respiratory tract cultures to be Burkholderia-free.

2013 IP\&C guideline, certainty: low

Sections in the text: IV.F.1

\section{Recommendations for CF Clinics and Other Ambulatory Care Settings}

In addition to the core recommendations, the CF Foundation recommends implementing the following recommendations in CF clinics and other ambulatory care areas, including those clinics where people with CF who have undergone lung or liver transplantation are followed.

\section{Scheduling in CF Clinics}

34. The CF Foundation recommends that CF clinics schedule and manage people with CF in ways to minimize time in common waiting areas. Such strategies include the following:

a. Stagger clinic schedule

b. Place people with CF regardless of their respiratory culture results in an exam room immediately on arrival to the clinic

c. Use a pager system or personal cell phone to alert people with CF that an exam room is available

d. Keep a person with CF in one exam room while the CF care team rotates through the exam room 
e. Do not share common items (eg, clinic computer and toys), and request that people with $\mathrm{CF}$ bring their own recreational items to clinic appointments

Source of supporting evidence: 2003 CF IP\&C guideline, Category II

2013 CF IP\&C guideline consensus: 100\%

Sections in the text: II; III.D.2; IV.F.1, 2, 7

35. The CF Foundation recommends that infants under 2 years of age be separated from other people with CF in CF clinics until adequate infection control education has been provided to and is understood by the caregivers.

Source of supporting evidence: 2009 CF Foundation guideline Management of Infants with CF Consensus, certainty: low; benefit: moderate

Sections in the text: II; III.A; IV.B; IV.F.1, 2, 7

36. The CF Foundation recommends that all newly diagnosed people with $\mathrm{CF}$ be separated from other people with $\mathrm{CF}$ in $\mathrm{CF}$ clinics until adequate IP\&C education has been provided to and is understood by newly diagnosed individuals and their caregivers.

2013 CF IP\&C guideline consensus: 100\%

Sections in the text: II; III.A; IV.B; IV.F.1, 2

37. The CF Foundation concludes that there is insufficient evidence at the time of publication of this document for or against routinely scheduling CF clinics on the basis of specific pathogens isolated from respiratory tract cultures.

2013 CF IP\&C guideline, certainty: low

Sections in the text: III.A; IV.F.1

\section{Pulmonary Function Testing}

38. The CF Foundation recommends that PFTs be performed in one of the following ways:

a. In the exam room at the beginning of the clinic visit

b. In a negative-pressure room (AIIR)

c. In a PFT laboratory with either portable or integrated HEPA filters

d. In a PFT laboratory without HEPA filtration, allowing 30 minutes to elapse before the next person with CF enters the PFT laboratory

2013 IP\&C guideline consensus: $100 \%$

Sections in the text: II.E; III.D.2; IV.A; IV.F.6

\section{Environmental Practices}

39. The CF Foundation recommends that exam rooms be cleaned and disinfected between patients using a 1-step process and Environmental Protection Agency (EPA)-registered hospital-grade disinfectant/detergent designed for housekeeping in accordance with institutional IP\&C policies.
Source of supporting evidence: 2003 CF IP\&C guideline, Category IB

2013 CF IP\&C guideline consensus: $100 \%$

Sections in the text: III.D.2; IV.F.7

\section{Designing a New CF Clinic}

40. The CF Foundation recommends that the leadership staff of CF centers collaborate with the institutional IP\&C and planning design and construction departments when designing a new CF clinic to ensure a design that includes the following:

a. Provision for management of people with CF who require Airborne Precautions

b. Appropriate number of exam rooms

c. Single-person restrooms

d. Adequate space for personal protective equipment (eg, masks, gowns, and gloves) at the point of use

2013 CF IP\&C guideline consensus: $100 \%$

Sections in the text: II.E; III.D.2; IV.F; IV.G

\section{Recommendations for Inpatient Settings}

In addition to the core recommendations, the CF Foundation recommends implementing the following recommendations in inpatient settings, including those units where people with $\mathrm{CF}$ who have undergone lung or liver transplantation are located.

\section{Room Placement}

41. The CF Foundation recommends that people with CF be placed in a single-patient room. Only people with CF who live in the same household may share a hospital room.

Source of supporting evidence: 2003 CF IP\&C guideline, Category II; 2006 MDRO guideline, Category IB

2013 CF IP\&C guideline consensus: 100\%

Sections in the text: II; IV.G

42. The CF Foundation recommends placing people with $\mathrm{CF}$ who are solid-organ transplant recipients in a singlepatient room in accordance with institutional policy and national guidelines. There is insufficient evidence to recommend for or against Protective Environment (ie, positive pressure room and HEPA filtration) for solid-organ recipients.

Source of supporting evidence: 2003 CF IP\&C guideline, Category II; 2007 transmission guideline, no recommendation, unresolved issue

2013 CF IP\&C guideline consensus: $100 \%$

Sections in the text: II; IV.G

\section{Practices for People with CF and Their Families}

43. The CF Foundation recommends evaluating people with CF on a case-by-case basis in accordance with institutional IP\&C policies for participation in activities outside the 
hospital room (eg, walking in the hallway, going to the playroom, physical therapy, exercise room, or school room) only when no other person with $C F$ is present and under the supervision of a trained staff member.

Considerations include the capability of a person with CF to contain his or her respiratory tract secretions, age, endemic levels of pathogens in an individual center, and adherence to the following practices:

a. Perform hand hygiene and put on a mask immediately before leaving patient rooms

b. After a person with CF has left a hospital activity room, clean surfaces and touched items with an EPA-registered hospital disinfectant/detergent

Source of supporting evidence: 2003 CF IP\&C guideline, Category IB/II

2013 CF IP\&C guideline consensus: $100 \%$

Sections in the text: II; IV.E.1; VI

44. The CF Foundation recommends that all people with CF perform all respiratory interventions (eg, aerosol therapy, airway clearance, and collection of respiratory tract cultures) in the patients' rooms. If 2 people with CF who live in the same household are sharing a room, these procedures should be performed when the second person is not in the room, whenever possible.

Source of supporting evidence: 2003 CF IP\&C guideline, Category IB

2013 CF IP\&C guideline consensus: $100 \%$

Sections in the text: II; II.C; II.D; II.E; III.D.2

45. The CF Foundation recommends that airway clearance devices (eg, flutter, acapella, pep device, and therapy vest) be for single-patient use only, in accordance with institutional IP\&C policies.

Source of supporting evidence: 2003 CF IP\&C guideline, Category II

2013 CF IP\&C guideline consensus: $100 \%$

Sections in the text: III.D.2; IV.A; IV.G

46. The CF Foundation recommends following institutional IP\&C policies for the use of masks, gowns, and gloves by individuals who are visiting hospitalized people with CF.

Source of supporting evidence: 2007 transmission guideline, no recommendation, unresolved issue

2013 IP\&C guideline consensus: $100 \%$

Sections in the text: II; IV.D.1; IV.D.2

\section{Care of Nebulizers in the Hospital}

47. The CF Foundation recommends the following:

a. Nebulizers are for single-patient use only

b. Aseptic technique is always followed when handling the nebulizer and dispensing medications

c. Single-dose vials of medication used in nebulizers are always preferred

d. Handheld disposable nebulizers are managed as follows:

i. After each use, rinse out residual volume with sterile water and wipe mask/mouthpiece with an alcohol pad

ii. Discard the nebulizer every 24 hours

e. Handheld reusable nebulizers (eg, home equipment) are managed as follows:

i. After each use, clean, disinfect, rinse with sterile water (if applicable, following cold disinfection method), and air dry away from sink

ii. After each use, the nebulizer can be reprocessed (eg, by steam sterilization) if the reprocessing is performed according to the manufacturer's instructions and the CF Foundation recommendations for home care (rec. 59) and if the nebulizer can be returned to the patient in time for the next treatment

Source of supporting evidence: 2003 CF IP\&C guideline, Category II; 2003 pneumonia guidelines, Category IB; 2008 ster-

ilization and disinfection guidelines, Category $I B$

2013 CF IP\&C guideline consensus: 100\%

Sections in the text: III.D.2; IV.E.2

\section{Animals}

48. The CF Foundation recommends that people with CF can participate in animal-assisted ("pet") therapy in accordance with institutional policies.

Source of supporting evidence: 2003 environmental guideline, Category II

2013 CF IP\&C guideline consensus: 100\%

Sections in the text: III.C

\section{Designing New Inpatient Facilities}

49. The CF Foundation recommends that the leadership staff of CF centers collaborate with the institutional IP\&C and the planning, design, and construction departments when designing a new inpatient unit to ensure a design that

a. Provides an adequate number of single-patient rooms to care for people with $\mathrm{CF}$

b. Includes a provision for people with CF who require possible Airborne Precautions

c. Provides access to exercise during hospitalization (eg, adequate space for exercise equipment)

d. Provides adequate space for personal protective equipment (eg, masks, gowns, and gloves) at the point of use

2013 CF IP\&C guideline consensus: 100\%

Sections in the text: I.C.5; II.D; IV.D.1; IV.D.2; IV.G; VI

\section{Recommendations for Nonhealthcare Settings}

In addition to the core recommendations, the CF Foundation recommends implementing the following recommendations in nonhealthcare settings. 


\section{Families with More than 1 Person with CF}

50. The CF Foundation recommends that it is preferable that people with CF who live in the same household perform airway clearance with only 1 person with $\mathrm{CF}$ in the room during treatment.

Source of supporting evidence: 2003 CF IP\&C guideline, Category II

2013 CF IP\&C guideline consensus: $100 \%$

Sections in the text: II; II.C; II.D; II.E

\section{Events and Activities}

51. The CF Foundation recommends against CF-specific camps or CF-specific educational retreats for groups of people with CF. Only 1 individual with CF should attend any camp or educational retreat unless they live in the same household.

However, family members who do not have CF may attend educational retreats. People with CF are encouraged to participate in camps and sports with non-CF individuals.

Source of supporting evidence: 2003 CF IP\&C guideline, Category $I B$

2013 CF IP\&C guideline consensus: $100 \%$

Sections in the text: II; III.A; IV.I.1

52. People with $\mathrm{CF}$ and their parents or legal guardians are not obligated to disclose the diagnosis of $\mathrm{CF}$ or the results of respiratory tract cultures to school or day care personnel. However, the CF Foundation recommends disclosure so that school or day care personnel can be made aware of the importance of IP\&C principles and practices for the protection of students with CF and can make the recommended accommodations. Such information must be maintained as confidential medical information unless the person with CF and/ or parent or legal guardian choose to make this information known.

Source of supporting evidence: 2003 CF IP\&C guideline, Category II

2013 CF IP\&C guideline consensus: 95\%

Sections in the text: II; III.A; IV.I.4

53. The CF Foundation recommends that people with CF attending the same day care and/or school should not be in the same room at the same time unless they live in the same household. The CF Foundation recommends education of day care/school personnel on the principles of IP\&C for CF so they can work with people with $\mathrm{CF}$ and/or parents or legal guardians to develop strategies to minimize contact between people with CF (eg, assignment to separate classrooms and separation during other scheduled common activities, including lunch, physical education, and recess).

Source of supporting evidence: 2003 CF IP\&C guideline, Category II

2013 CF IP\&C guideline consensus: $100 \%$

Sections in the text: IV.I.4
54. The CF Foundation recommends that only 1 person with CF attend CF Foundation-sponsored, healthcare-sponsored, or CF center-sponsored indoor events (eg, CF Education Days) unless they live in the same household, to reduce the risk of person-to-person transmission of CF pathogens.

2013 CF IP\&C guideline consensus: $100 \%$

Sections in the text: II; III.A; IV.I.2

55. The CF Foundation recommends developing and utilizing alternative $\mathrm{CF}$ education programs, (eg, videotapes, video conferencing, $\mathrm{CD}-\mathrm{ROM}$ web-based learning, and apps) that do not require face-to-face meetings among people with CF.

Source of supporting evidence: 2003 CF IP\&C guideline, Category II

2013 CF IP\&C guideline consensus: $100 \%$

Sections in the text: IV.B; IV.I.1; IV.I.2; VI

56. The CF Foundation recommends that people with CF can attend CF Foundation-sponsored, healthcare-sponsored, or CF center-sponsored outdoor events (eg, Great Strides) providing they maintain a distance of at least 6 feet ( 2 meters) from others with CF.

2013 CF IP\&C guideline consensus: 100\%

Sections in the text: II; III.A; IV.I.3

\section{MRSA}

57. The CF Foundation recommends that people with CF should avoid direct contact with people with skin and softtissue infections caused by MRSA unless wounds are covered, hand hygiene is performed frequently, personal items (eg, towels) are not shared, sports equipment is cleaned between use, and cleaning protocols for environmental surfaces are established to reduce the risk of MRSA transmission.

Source of supporting evidence: CDC guidance (http:// www.cdc.gov/mrsa/prevent/personal.html)

2013 CF IP\&C guideline consensus: $100 \%$

Sections in the text: III.B.2

58. The CF Foundation recommends that people with CF and respiratory cultures positive for MRSA should not be restricted from contact with people without CF in congregate settings (eg, sports teams, classrooms, and the workplace) if the person with CF performs appropriate hand and respiratory hygiene.

Source of supporting evidence: CDC guidance (http:// www.cdc.gov/mrsa/prevent/personal.html)

2013 CF IP\&C guideline consensus: $100 \%$

Sections in the text: III.A.4; III.B.2

\section{Nebulizers: Cleaning and Disinfecting}

59. The CF Foundation recommends that the following steps be performed for nebulizers used in the home as soon as possible after each use: 
a. Clean the nebulizer parts with dish detergent soap and water

b. Disinfect the nebulizer parts using one of the following methods:

Heat methods:

a. Place in boiling water and boil for 5 minutes

b. Place in a microwave-safe receptacle submerged in water and microwave for 5 minutes

c. Use a dishwasher if the water is more than or equal to $70^{\circ} \mathrm{C}$ or $158^{\circ} \mathrm{F}$ for 30 minutes

d. Use an electric steam sterilizer

Cold methods:

a. Soak in $70 \%$ isopropyl alcohol for 5 minutes

b. Soak in 3\% hydrogen peroxide for 30 minutes

$i$. Rinse off the cold-method disinfectant using sterile water, not tap water; the final rinse must be with sterile or filtered (less than or equal to 0.2 -micron filter) water

ii. Air dry the nebulizer parts before storage

Source of supporting evidence: 2003 CF IP\&C guideline, Category II

2013 CF IP\&C guideline consensus: $100 \%$

Sections in the text: III.D.1; IV.E.3

60. The CF Foundation recommends that nebulizers used in the home should not be disinfected with acetic acid (vinegar), bleach solutions, or benzalkonium chloride (eg, "Control III").

2013 CF IP\&C guideline consensus: $100 \%$

Sections in the text: IV.E.3

\section{Leisure Activities}

61. The CF Foundation recommends that people with CF should limit prolonged and/or repeated exposure to activities that generate dust from soil and organic matter (eg, gardening and lawn mowing) to decrease exposure to potential soilborne pathogens (eg, Burkholderia spp. and Aspergillus spp.).

2013 CF IP\&C guideline consensus: $100 \%$

Sections in the text: IV.D.1

62. The CF Foundation recommends that people with CF should avoid exposure to construction and renovation activities that generate dust to decrease exposure to potential pathogens (eg, Aspergillus spp.).

2013 CF IP\&C guideline consensus: $100 \%$

Sections in the text: III.D.1; III.D.3

63. The CF Foundation recommends that people with CF can swim in pools or water parks with adequate disinfection (eg, chlorination).

2013 CF IP\&C guideline consensus: $100 \%$

Sections in the text: III.D.1
64. The CF Foundation recommends that people with CF avoid activities in hot tubs, whirlpool spas, and stagnant water.

2013 CF IP\&C guideline consensus: 100\%

Sections in the text: III.D.1

65. There is insufficient evidence at the time of publication of this document for the CF Foundation to recommend for or against people with $\mathrm{CF}$ avoiding activities in natural bodies of water that are not stagnant (eg, ocean, ponds, and hot springs).

2013 CF IP\&C guideline, certainty: low

Sections in the text: III.D.1

\section{Contact with Pets or Farm Animals}

66. The CF Foundation recommends that people with CF perform hand hygiene after changing the litter, handling feces, cleaning and disinfecting the cages or fish tanks of their pets, or interacting with farm animals.

Source of supporting evidence: http://www.cdc.gov /healthypets/

2013 CF IP\&C guideline consensus: $100 \%$

Sections in the text: III.C

67. The CF Foundation recommends that people with CF avoid cleaning stalls, pens, or coops.

2013 CF IP\&C guideline consensus: 100\%

Sections in the text: III.C

\section{Recommendations for Healthcare Personnel WITH CF}

In addition to the core recommendations, the CF Foundation recommends implementing the following recommendations for healthcare personnel with CF.

68. The CF Foundation recommends that healthcare personnel with CF should not provide care for other people with CF.

\section{CF IP\&C guideline consensus: $100 \%$}

Sections in the text: III.A; V

69. The CF Foundation recommends that people with CF interested in a career in healthcare receive counseling from their CF care team regarding specialty areas wherein job duties minimize the risk of transmission or acquisition of potential pathogens.

Source of supporting evidence: 2003 CF IP\&C guideline, Category II

2013 CF IP\&C guideline consensus: $100 \%$

Sections in the text: III; V

70. The CF Foundation recommends that healthcare personnel with CF consider informing their employers' workforce health and safety department about their diagnosis of CF to ensure that job duties are assigned and care practices 
are adopted that minimize the risk of acquisition or transmission of potential pathogens. This disclosure is legally required to be kept confidential.

2013 CF IP\&C guideline consensus: 100\%

Sections in the text: $\mathrm{V}$

71. The CF Foundation recommends that when it is known that a healthcare provider with or without CF is infected/ colonized with MRSA, work assignments should be made according to local hospital policy.

Source of supporting evidence: 2003 CF IP\&C guideline, Category II

2013 CF IP\&C guideline consensus: $100 \%$

Sections in the text: V

72. The CF Foundation recommends that healthcare personnel with CF be assigned to care for patients without CF on a case-by-case basis, considering health- and behaviorrelated factors, such as

a. Frequency and severity of coughing episodes, quantity of sputum production during these episodes, and ability to contain respiratory tract secretions;

b. Ability to use barrier precautions and adhere to IP\&C guidelines, Centers for Medicare \& Medicaid Services, HICPAC, and CDC guidelines; and

c. Risk of transmission of pathogens by healthcare personnel with $\mathrm{CF}$ in the context of specific job duties.

Source of supporting evidence: 2003 CF IP\&C guideline, Category II

2013 CF IP\&C guideline consensus: $100 \%$

Sections in the text: III.A; V

ViI. Recommendations for Psychosocial and Medical IMPACT OF IP\&C

The CF Foundation recommends implementing the following recommendations to reduce the psychosocial impact of IP\&C for people with CF, their families, and healthcare personnel.

73. The CF Foundation recommends educating, when appropriate, friends, teachers, employers, and coworkers about the rationale for the IP\&C recommendations.

Source of supporting evidence: 2003 CF IP\&C guideline, Category II

2013 CF IP\&C guideline consensus: $100 \%$

Sections in the text: IV.B; VI

74. The CF Foundation recommends identifying CF centerspecific concerns for the potential psychosocial impact of the IP\&C guideline for people with CF in the hospital, clinic, community, school, and home and strategies, including an available counselor, to minimize the negative impact.

Source of supporting evidence: 2003 CF IP\&C guideline, Category II

2013 CF IP\&C guideline consensus: $100 \%$

Sections in the text: VI
75. The CF Foundation recommends that the CF care team inform people with $\mathrm{CF}$ and their parents or legal guardians of their microbiologic status. People with CF and their parents or legal guardians will then determine whom they will inform.

Source of supporting evidence: 2003 IP\&C guideline, Category II

2013 IP\&C guideline consensus: 100\%

Sections in the text: IV.I.1; IV.I.4; VI

76. The CF Foundation recommends collaboration with the child life staff to ensure individualized programs consistent with the recommended IP\&C guidelines.

Source of supporting evidence: 2003 CF IP\&C guideline, Category II

2013 CF IP\&C guideline consensus: $100 \%$

Sections in the text: IV.B; VI

77. The CF Foundation recommends making accommodations (eg, providing entertainment, enhancing communication with the outside world, facilitating visits with non-CF individuals, and adapting child life programs) to relieve the psychosocial stress of inpatient and outpatient IP\&C guidelines without placing people with $\mathrm{CF}$ at risk for transmission or acquisition of pathogens.

Source of supporting evidence: 2003 CF IP\&C guideline, Category II

2013 CF IP\&C guideline consensus: $100 \%$

Sections in the text: VI

\section{BACKGROUND INFORMATION SUPPORTING} THE RECOMMENDATIONS

\section{CF Microbiology and Molecular Typing}

\section{I.A. General Microbiology Methods}

The 2003 Infection Control Guideline for CF provided detailed recommendations for obtaining and processing $\mathrm{CF}$ respiratory tract specimens that were endorsed by the American Society for Microbiology and the National Committee for Clinical Laboratory Standards (now the Clinical and Laboratory Standards Institute). ${ }^{1}$ Others have supported these recommendations. ${ }^{39,40}$ Furthermore, review of the protocols of clinical microbiology laboratories in the United States obtained in 2003-2004 demonstrated excellent adherence to the 2003 Infection Control Guideline for CF recommendations for processing CF specimens. ${ }^{41}$ Thus, the majority of clinical laboratories processing the CF respiratory tract specimens have standardized their techniques, use appropriate selective media and prolonged incubation, and identify gram-negative organisms to the species level. The CF Foundation recommends continued use of the methods described in the 2003 Infection Control Guideline for CF for when to perform respiratory tract cultures, how to transport specimens, and the use of selective media. ${ }^{1}$ A detailed description of processing lower respiratory tract specimens for NTM will be provided 
in a joint CF Foundation and ECFS guideline (B.C.M., written personal communication, April 2013).

The committee reviewed the following microbiology topics but agreed not to develop revised recommendations in the updated guidelines: (1) The relative merits of different types of respiratory tract specimens. The positive and negative predictive values of deep throat specimens or oropharyngeal specimens for the lower airway specimens have been studied with varying results, presumably due to the different patient populations and pathogens studied. ${ }^{42}$ The yield of induced sputum relative to upper airway specimens has also been assessed in research settings, and induced sputum generally yields more potential pathogens. ${ }^{43}$ The 2003 Infection Control Guideline for CF recommendations for processing all types of respiratory tract specimens (throat, sputum, or bronchoalveloar lavage) continues to be appropriate. (2) The relative merits of different frequencies of respiratory tract cultures. Cultures of the respiratory tract can detect new pathogens, guide therapy, monitor the success of eradication strategies, and distinguish transient versus persistent colonization/infection. However, more frequent surveillance is associated with increased incidence and prevalence of CF pathogens. ${ }^{44}$ The 2003 Infection Control Guideline for CF recommendation for quarterly cultures-or more frequently if clinically indicated - continues to be supported by published studies. ${ }^{45}$ (3) The role played by matrix-assisted laser desorption ionization time-of-flight (MALDI-TOF) mass spectrometry platforms for identification of gram-negative bacilli from patients with CF. Several studies have found an advantage of this technology for rapid identification of pathogens that require many days using traditional phenotypic and genomic sequencing methods. ${ }^{46-48}$ However, this technology is not widely available in the United States. (4) The relative merits of susceptibility testing to guide treatment of CF pathogens. Recent studies have questioned the clinical utility of performing routine susceptibility testing. ${ }^{49,50}$ Nonetheless, antimicrobial susceptibility can distinguish methicillin-susceptible $S$. aureus (MSSA) from MRSA, identify unique multidrug-resistance patterns, and has been crucial for epidemiologic investigations. For example, CF clinicians were alerted to the transmission of epidemic strains of $P$. aeruginosa due to the emergence of ceftazidime-resistant, ${ }^{51}$ colistin-resistant, ${ }^{52}$ or multidrug-resistant strains. ${ }^{30,53}$ Thus, susceptibility testing is still recommended as per the 2003 Infection Control Guideline for CF. (5) Clinical implications of the CF microbiome. The reader is referred to several recent studies for information on this increasingly important topic. ${ }^{54-57}$

\section{I.B. Molecular Typing Methodologies}

\section{Overview}

While earlier methods for typing bacteria from the respiratory tract of individuals with CF for epidemiologic purposes were based primarily on comparison of phenotypic (physical) features, molecular methods using analysis of the genetic content of bacteria are now preferred. Compared with phenotypic methods, genotyping methods are more reproducible and provide greater discriminatory power in differentiating epidemiologically related strains from unrelated strains. Further attributes of the ideal genotyping system include ease of use, low cost, and unambiguous interpretation. ${ }^{58-60}$

\section{Random-Amplified Polymorphic DNA (RAPD)}

RAPD typing is based on PCR amplification of random sections of the bacterial genome. ${ }^{61}$ The amplified DNA segments are separated by gel electrophoresis, and the resulting banding pattern is compared with that of other bacteria visually or by means of computer-imaging software. Bacterial isolates with a high level of similarity in RAPD pattern are considered indistinguishable or highly likely to be the same strain.

\section{Repetitive-Element PCR (rep-PCR)}

Another PCR-based genotyping method relies on the amplification of certain repetitive genetic elements found within the bacterial genome. A frequently used target for such repPCR typing is a genetic element referred to as the BOX A1R element (so-called BOX-PCR typing). ${ }^{62}$ As with RAPD, the DNA banding patterns of bacterial isolates revealed by repPCR are compared; those with a highly similar pattern are considered highly likely to be related.

\section{Pulsed-Field Gel Electrophoresis (PFGE)}

PFGE has been a mainstay of bacterial genotyping for the past 2 decades. PFGE evaluates genetic polymorphisms within the entire bacterial genome by macrorestriction, a technique that extracts genomic DNA from bacterial cells and then cleaves the DNA into large fragments using a restriction enzyme. These DNA fragments are separated by size using gel electrophoresis. The resulting banding pattern is compared among bacterial isolates; those with highly similar patterns are considered highly likely to be related. ${ }^{60}$

\section{Multilocus Sequence Typing (MLST)}

MLST has become a preferred method for bacterial genotyping. ${ }^{63}$ This method measures DNA sequence variations in a set of housekeeping genes that are present in all strains of a given species and characterizes strains by their unique allelic profiles. For each housekeeping gene of interest, the different sequences found within a bacterial species are designated as distinct alleles. For each isolate, the alleles identified for each of the housekeeping genes define the allelic profile or sequence type (ST). Compared with PFGE and PCR-based genotyping methods, MLST has distinct advantages, as it yields unambiguous, reproducible results that can be compared between laboratories. Public-access ST databases make MLST particularly well suited to global studies of the epidemiology of CF pathogens. ${ }^{64}$ 


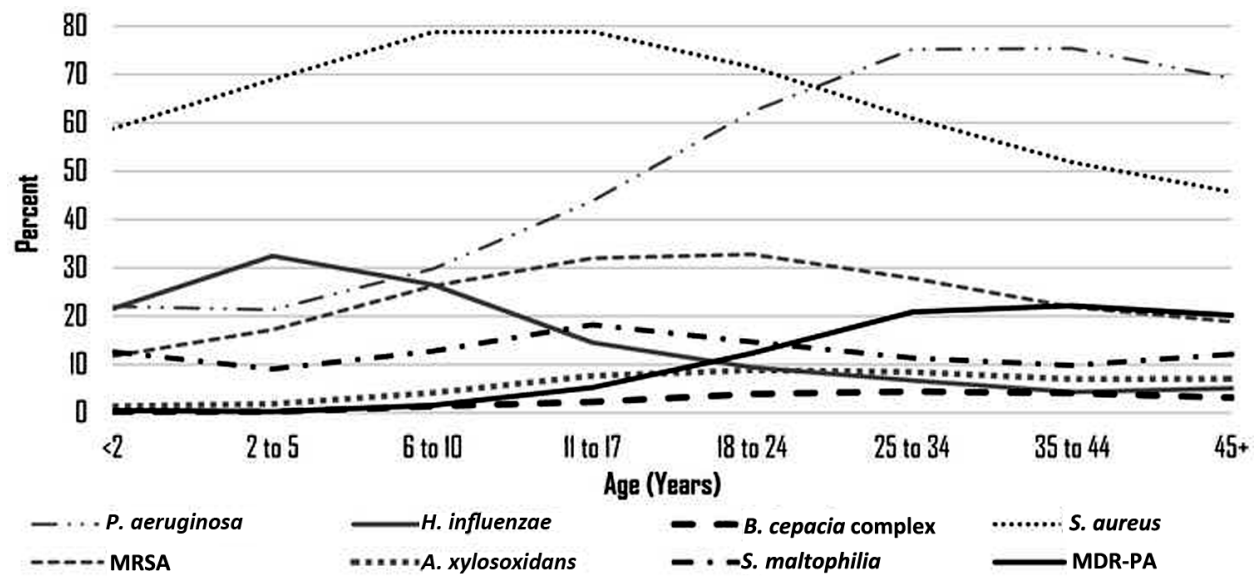

FIGURE 1. Age-specific prevalences of respiratory organisms, 2012. Age-specific prevalences of Pseudomonas aeruginosa, methicillin-resistant Staphylococcus aureus (MRSA), Haemophilus influenzae, Achromobacter xylosoxidans, Burkholderia cepacia complex, Stenotrophomonas maltophilia, Staphylococcus aureus, and multidrug-resistant Pseudomonas aeruginosa (MDR-PA) among patients with cystic fibrosis in the United States in 2012 are shown. These data reflect an analysis of the US Cystic Fibrosis Foundation Patient Registry.

\section{Whole-Genome Sequencing}

Most recently, whole-genome sequencing has been used to help define the epidemiology of bacterial pathogens for which conventional molecular typing may not have the resolution to accurately analyze population structure. Notably, wholegenome sequencing was used to assess transmission of NTM between individuals with and without $\mathrm{CF}^{65,66}$ The slow mutation rate of NTM is another reason for the need for wholegenome sequencing methods with these pathogens.

\section{Summary}

Several molecular typing strategies have been developed that have been instrumental in documenting shared strains of CF pathogens and identifying potential environmental sources, as described below. It is likely that whole-genome sequencing will become more widely used to delineate the epidemiology of some pathogens, such as NTM and MRSA, while other strategies, such as MLST, will continue to be used for Burkholderia and Pseudomonas spp. National CF organizations in Canada, the United States, and several countries in the European Union have established research and referral laboratories for molecular typing of CF bacterial isolates that interact with one another through international networks, such as the International Burkholderia cepacia Working Group. ${ }^{67}$ Such interactions help determine whether bacterial strains are found in more than 1 country, and thanks to the efforts of these laboratories, our understanding of the molecular epidemiology of CF pathogens has greatly expanded, as described further below.

In addition to its use in outbreak investigations, molecular typing is a critical tool in active surveillance programs and in monitoring the success of IP\&C strategies. It should be emphasized, however, that molecular typing is best used to augment conventional shoe-leather epidemiology, since most genotyping methods are not performed routinely in diagnostic clinical microbiology laboratories and active surveillance using molecular methods is not a component of routine CF care in the United States.

\section{I.C. Epidemiology of CF Pathogens}

\section{I.C.1. Overview}

The CF Foundation Patient Registry (CFFPR) is an invaluable source of data to further our understanding of the epidemiology of CF pathogens. The CFFPR has improved data collection for CF microbiology by creating numerous dropdown menus for both common and emerging pathogens. The CF Foundation provides annual data on the epidemiology of CF pathogens, as shown for 2012 in Figure $1 .^{68}$ S. aureus is the most common CF pathogen in the first 2 decades of life. While MSSA is more prevalent than MRSA, the prevalence of MRSA is highest in 11-24-year-olds. P. aeruginosa is detected in more than $20 \%$ of young infants, and nearly $80 \%$ of adults are infected with this pathogen. The prevalence of multidrug-resistant gram-negative organisms, including Stenotrophomonas maltophilia, Achromobacter xylosoxidans, and B. cepacia complex, increases with age.

The CF Foundation analyzed the changing prevalence of CF pathogens from 1988 to 2012, as shown in Figure 2. ${ }^{68}$ Several pathogens, including MRSA, S. aureus, and S. maltophilia, have increased during this time. The explanation for these increases is unknown. It is likely that improved microbiology laboratory processing and data collection have improved our ability to detect and report these microorganisms, but increasing longevity, antimicrobial selective pressure, and potentially person-to-person transmission may also contribute to these findings. In contrast, the prevalence of $P$. aeruginosa and B. cepacia complex has decreased, which suggests 


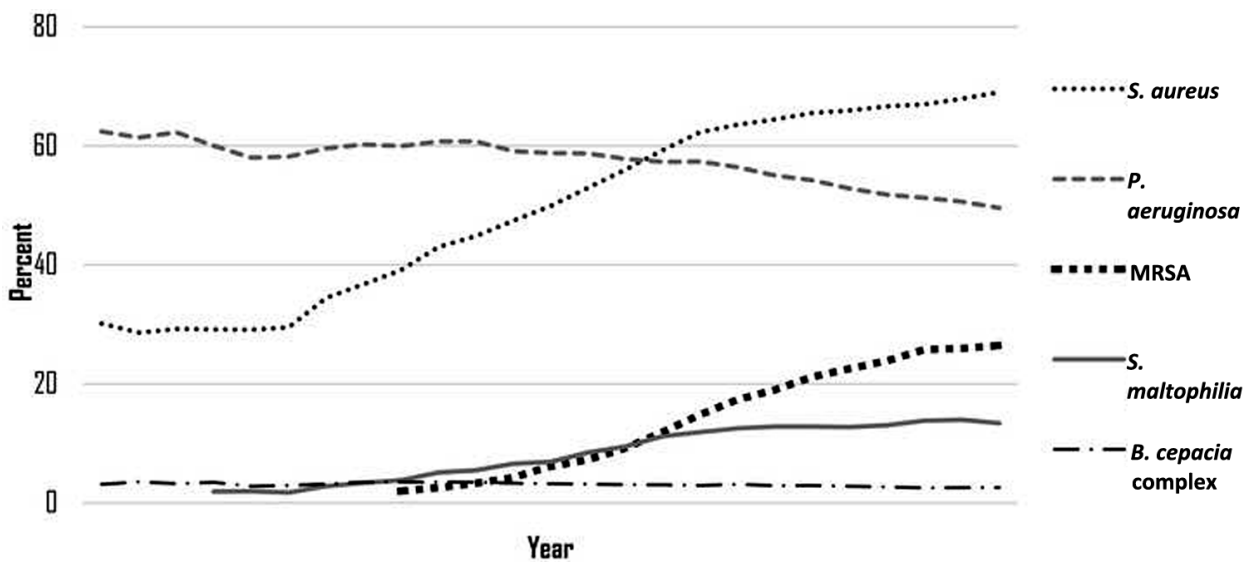

FIGURE 2. Respiratory organism prevalences, 1988-2012. Prevalences of Staphylococcus aureus, Pseudomonas aeruginosa, methicillin-resistant Staphylococcus aureus (MRSA), Stenotrophomonas maltophilia, and Burkholderia cepacia complex among patients with cystic fibrosis in the United States of all ages from 1988 to 2012 are shown. These data reflect an analysis of the US Cystic Fibrosis Foundation Patient Registry.

that treatment paradigms, including early eradication strategies for $P$. aeruginosa and improved IP\&C, may have influenced the epidemiology of these CF pathogens. The following sections provide a brief overview of the epidemiology of selected CF pathogens. Potential sources of these pathogens, including person-to-person transmission, are discussed in Section III.

\section{I.C.2. Burkholderia spp.}

At present, the B. cepacia complex consists of 18 distinct yet closely related species (Table 4). ${ }^{69-80}$ The frequency of detection of these species in people with CF varies considerably, although Burkholderia cenocepacia and Burkholderia multi-

TAвLE 4. Burkholderia cepacia Complex

\begin{tabular}{lcll}
\hline Species name & $\begin{array}{c}\text { Former } \\
\text { genomovar } \\
\text { designation }\end{array}$ & $\begin{array}{c}\text { Year } \\
\text { identified } \\
\text { and/or named }\end{array}$ & Reference(s) \\
\hline B. cepacia & I & 1950,1997 & 69,74 \\
B. multivorans & II & 1997 & 74 \\
B. cenocepacia & III & 1997,2003 & 73,74 \\
B. stabilis & IV & 1997,2000 & 74,75 \\
B. vietnamiensis & V & 1995,1997 & 74,80 \\
B. dolosa & VI & 2001,2004 & 79 \\
B. ambifaria & VII & 2001 & 70 \\
B. anthina & VIII & 2002 & 71 \\
B. pyrrocinia & IX & 2002 & 71 \\
B. ubonensis & $\ldots$ & 2000,2008 & 76,77 \\
B. latens & $\ldots$ & 2008 & 76 \\
B. diffusa & $\ldots$ & 2008 & 76 \\
B. arboris & $\ldots$ & 2008 & 76 \\
B. seminalis & $\ldots$ & 2008 & 76 \\
B. metallica & $\ldots$ & 2008 & 76 \\
B. contaminans & $\ldots$ & 2009 & 72 \\
B. lata & $\ldots$ & 2009 & 72 \\
B. pseudomultivorans & $\ldots$ & 2013 & 78 \\
\hline
\end{tabular}

vorans are most common. ${ }^{26,81}$ Burkholderia gladioli is the third most frequently isolated Burkholderia species among CF patients in the United States. ${ }^{26}$ However, while B. gladioli are phenotypically quite similar to the species in the $B$. cepacia complex, it is not a member of the B. cepacia complex. ${ }^{82}$

\section{I.C.3. Other Gram-Negative Species}

The Burkholderia Reference Laboratory and Repository at the University of Michigan has expanded our understanding of the epidemiology of less commonly isolated gram-negative organisms in CF, including Ralstonia spp. (eg, R. picketti and R. paucula) and Pandoraea spp. ${ }^{26}$ Readers are referred to several excellent reviews that have highlighted the recent epidemiology of CF pathogens. ${ }^{26,81,83}$

\section{I.C.4. Small Colony Variant (SCV) S. aureus}

In the past several years, there has been increasing interest in the clinical and therapeutic implications of SCVs of $S$. aureus in CF. S. aureus persists in the airways of people with CF for years and can develop a hypermutator phenotype that promotes adaptive changes, including SCVs, thought to facilitate survival of this organism within the CF airway. ${ }^{84,85}$ SCVs are detected visually on laboratory agar plates and exhibit slower growth rates due to metabolic defects (eg, thymidine biosynthesis deficiency ${ }^{86}$ ), and they therefore require special susceptibility testing. However, the testing method has not been standardized by the Clinical and Laboratory Standards Institute.

Several potential clinical implications of SCV strains have been proposed. SCV strains of $S$. aureus are thought to have increased resistance to the innate immune system and increased resistance to antibiotics. ${ }^{85,87,88}$ In vitro, SCVs have an increased ability to infect normal and CF airway epithelial cells. ${ }^{89}$ In non-CF patients, SCV S. aureus are associated with 
chronic or recurrent infections, such as endocarditis and osteomyelitis. $^{86,90}$

SCV strains have been isolated from $8 \%$ to $33 \%$ of individuals with $\mathrm{CF}$ who are infected with $S$. aureus. ${ }^{91-93}$ SCV $S$. aureus are associated with older age, coinfection with P. aeruginosa, lower lung function, ${ }^{92}$ and treatment with antibiotics, specifically trimethoprim-sulfamethosoxazole. In vitro, SCV strains can be induced by exoproducts expressed by $P$. aeruginosa. ${ }^{94}$

Recently, a causal relationship was suggested between the emergence of SCV S. aureus and a decline in lung function. In a CF center in the United States, 100 children with CF, of whom 24 had SCV S. aureus, were followed for an average of 1.7 years. ${ }^{95}$ Those with SCV strains had lower lung function at the beginning and end of the study, but they had a similar rate of pulmonary exacerbations. Notably, $33 \%$ of the children with SCV strains did not have normal-colony S. aureus strains, which suggests that these children would not have been identified as infected with $S$. aureus had SCV strains not been sought. ${ }^{95}$ In addition, 2 pairs of subjects had the same SCV strains, suggesting possible transmission of SCVs between children with CF.

Currently, it is unknown how many clinical laboratories have instituted methods to detect SCV strains of S. aureus, and there are no standardized methods for detection and susceptibility testing. Additional studies are needed to further describe the epidemiology of SCV S. aureus as well as the treatment and IP\&C implications for CF. Furthermore, SCVs of other bacteria, including Pseudomonas, ${ }^{93,96}$ Stenotrophomonas, ${ }^{97}$ and B. cepacia complex, ${ }^{98}$ have been described in people with $\mathrm{CF}$.

\section{I.C.5. Nontuberculous Mycobacteria}

Among the NTM are several opportunistic human pathogens, including Mycobacterium intracellulare and the species in the Mycobacterium avium complex (MAC; M. avium subsp. avium, M. avium subsp. silvaticum, and $M$. avium subsp. paratuberculosis). ${ }^{99}$ The rapid-growing NTM species include those in the M. abscessus complex. Although the taxonomy of the M. abscessus complex was uncertain at the time of publication, currently 3 closely related subspecies are described: M. abscessus subsp. abscessus, M. abscessus subsp. massiliense, and M. abscessus subsp. bolletii.$^{100,101}$

The prevalence of NTM in people with CF varies between countries and centers and appears to be increasing. The 2 most common species of NTM seen in individuals with CF are MAC and M. abscessus. Although MAC is more prevalent in North America, M. abscessus is more common in Europe and Israel. ${ }^{102-104}$ In France, MAC was detected in older patients with less severe disease. ${ }^{104}$ Thus, it appears that MAC and $M$. abscessus may target different subpopulations of people with CF.

\section{I.C.6. Aspergillus spp.}

People with CF are at increased risk for colonization with filamentous fungi. The most frequently identified filamentous fungi are Aspergillus fumigatus, Scedosporium apiospermum, and Aspergillus terreus. ${ }^{105}$ Less common fungi include Aspergillus flavus, Aspergillus nidulans, Exophilia dermatitidis, Scedosporium prolificans, Penicillium emersonii, and Acrophialophora fusispora. People with $\mathrm{CF}$ are at risk for allergic bronchopulmonary aspergillosis (ABPA), but many do not fulfill the clinical criteria for a diagnosis of ABPA. ${ }^{106-109}$ Furthermore, criteria for initiation of antifungal therapy in individuals with positive Aspergillus cultures are incompletely defined. ${ }^{110}$ In addition, invasive infection due to Aspergillus may occur, especially after lung transplantation. ${ }^{111}$

\section{I.D. Surveillance Strategies for CF Pathogens}

Routine real-time surveillance for epidemiologically significant microorganisms is recommended in acute care settings to understand endemic rates and to identify outbreaks as soon as possible. ${ }^{5}$ Regulatory requirements from state health departments and priorities established by local IP\&C departments determine specific surveillance strategies. Local clinical microbiology laboratories are crucial partners to ensure accurate and meaningful data. Examples of pathogens for which surveillance is performed in hospitalized patients without CF, under defined circumstances, include MRSA, vancomycinresistant enterococci (VRE), gram-negative bacilli resistant to carbapenem agents (CRE), and Clostridium difficile.

Routine surveillance for CF pathogens at individual CF centers can be used to track and trend the incidence and prevalence of specific microorganisms. Surveillance can assist centers in measuring the efficacy of both IP\&C measures and other treatment strategies, such as early eradication. As described above, molecular typing is an invaluable tool for assessing potential patient-to-patient transmission, but it is not yet available routinely. Surveillance data for particular species can be generated by local clinical microbiology laboratories or by the CFFPR.

The CF community is continually challenged by the changing epidemiology of CF pathogens. Not only have new pathogens emerged, but the epidemiology of classic CF pathogens has changed thanks to new treatment strategies (eg, early eradication of $P$. aeruginosa) and improved microbiologic detection and identification. To remain vigilant, surveillance strategies that assess the impact of therapeutic interventions, identify potential outbreaks, and monitor the success of IP\&C practices must be integrated into CF care.

\section{Routes of Transmission of CF Pathogens}

\section{II.A. Contact and Droplet Transmission}

Several routes of transmission have been described for CF pathogens, including direct contact with infectious respira- 
tory secretions (eg, by kissing), indirect contact with an intermediate object contaminated with infectious respiratory secretions (eg, hands, environmental surfaces, or shared equipment), and infectious droplets from the respiratory tract that can travel in the air a distance of 3-6 feet (1-2 meters). These routes of transmission are summarized in Table 5. Bacterial and viral pathogens can remain viable on hands or inanimate surfaces for minutes, hours, or even days. ${ }^{12,113}$

Since publication of the 2003 Infection Control Guideline for CF, several studies conducted in people with and without CF who are infected with viral or bacterial pathogens have expanded our understanding of droplet transmission and now challenge the 3-foot rule. These studies include epidemiologic data collected during outbreaks of influenza ${ }^{114,115}$ and SARS in non-CF individuals, ${ }^{116-118}$ experimental and observational studies performed in people with $\mathrm{CF}^{119-121}$ and studies of the dynamics of infectious aerosols. ${ }^{119,122,123}$

\section{II.B. Classic View of Droplet Transmission}

In the classic view of droplet transmission, infectious respiratory droplets (more than $5 \mu \mathrm{m}$ in diameter) are expelled by one person onto the mucous membranes of the nose, mouth, or conjunctivae of another susceptible person within 3 feet. ${ }^{124}$ Infectious droplets are generated by coughing, sneezing, or talking or during such procedures as suctioning, intubation, chest physiotherapy, or pulmonary function testing. Infectious droplets remain suspended in the air for a short time, generally minutes, and can contaminate horizontal environmental surfaces, equipment, and the hands of patients and healthcare personnel. Droplets are contrasted with much smaller droplet nuclei (less than $3.3 \mu \mathrm{m}$ in diameter), which can travel farther, remain suspended in the air for longer periods of time, do not require face-to-face contact for transmission, and are directly inhaled into the respiratory tract. Thus, pathogens transmitted by droplet nuclei do not require individuals to be in close proximity but do require them to share common air space.

\section{II.C. Emerging View of Droplet Transmission}

New data have challenged the classic view of droplet transmission. Infectious droplets containing influenza virus and SARS-CoV traveled 3-6 feet. ${ }^{6,114,116,118}$ Aerosols of droplet nuclei from patients infected with influenza can be generated during intubation and suctioning. Droplet size and distance traveled can be affected by (1) environmental factors (eg, humidity, temperature, air currents, and number of air changes per hour in a room), (2) agent factors (eg, infectious load, transferability, survivability, infectivity, and contagiousness), and (3) host factors (eg, susceptibility and behavior). ${ }^{122,123}$

In CF, several recent studies have explored the dynamics of droplet transmission. In an experimental model, subjects with CF infected with $P$. aeruginosa coughed into a chamber, and both droplets and smaller droplet nuclei containing viable organisms were collected as far as 6 feet from the subjects. ${ }^{121}$ CF pathogens were recovered from the air collected 6 feet from CF subjects who were performing PFTs, ${ }^{125}$ and P. aeruginosa was recovered from the air in hospital rooms, rooms after chest physiotherapy was performed, the hospital cor-

TAвLE 5. Modes of Transmission of Potential Pathogens in Cystic Fibrosis

\begin{tabular}{|c|c|c|c|}
\hline Type of transmission & Mode of transmission & $\begin{array}{l}\text { Examples of respiratory } \\
\text { tract pathogens }\end{array}$ & Source \\
\hline Contact transmission & $\begin{array}{l}\text { Direct or indirect contact } \\
\text { with infectious secretions }\end{array}$ & $\begin{array}{l}\text { MRSA } \\
\text { Pseudomonas aeruginosa } \\
\text { Burkholderia spp. } \\
\text { Respiratory syncytial virus }\end{array}$ & $\begin{array}{l}\text { Hands of healthcare workers } \\
\text { Shared toys } \\
\text { Contaminated respiratory therapy equipment } \\
\text { or surfaces }\end{array}$ \\
\hline
\end{tabular}

NOTE. MRSA, methicillin-resistant Staphylococcus aureus; SARS-CoV, severe acute respiratory syndrome coronavirus. 
тав LE 6. Relative Frequency of Shared Strains of Different Cystic Fibrosis Pathogens

\begin{tabular}{lcl}
\hline Species & Frequency $^{\mathrm{a}}$ & \multicolumn{1}{c}{ Reference(s) $^{-}$} \\
\hline Pseudomonas aeruginosa & +++ & $27,28,30,130-132$ \\
Burkholderia spp. & +++ & $27,133-135$ \\
Methicillin-susceptible Staphylococcus aureus & + & 136,137 \\
Methicillin-resistant S. aureus & ++ & 138,139 \\
Stenotrophomonas maltophilia & + & 140,141 \\
Achromobacter xylosoxidans & + & $142-144$ \\
Mycobacterium avium complex & None described & 102 \\
Mycobacterium abscessus subsp. massiliense & + & $66,129,145$ \\
\hline
\end{tabular}

${ }^{a}$ Frequency $(+$ to +++ ) is based on the relative number of published reports.

ridor, and a CF clinic. ${ }^{126,127}$ Factors associated with generating infectious droplets are unknown, as exacerbations, sick versus well CF clinic visits, and age were not predictive of the rate of recovery from air samples. ${ }^{125,128}$

\section{II.D. Potential Role of Droplet Nuclei}

Most recently, the potential for person-to-person droplet and/ or droplet nuclei transmission of M. abscessus subsp. massiliense has been suggested. ${ }^{66,129}$ Transmission in one center was halted by simultaneous implementation of multiple IP\&C strategies, including separation of people with CF infected with this pathogen, increased microbiologic surveillance, enhanced environmental cleaning, mask use by individuals with $\mathrm{CF}$, and the use of negative pressure rooms among adults with CF. ${ }^{129}$ (See Section III.A.5 for a more detailed description of NTM transmission.)

\section{II.E. Paradigm for Transmission of Respiratory Pathogens}

In an effort to explain observations made during the 2003 SARS epidemics, the following paradigm was proposed to describe the potential for transmission of respiratory tract pathogens by both infectious droplets and droplet nuclei under different conditions: ${ }^{117}$ (1) Obligate transmission is that which occurs under natural conditions (eg, transmission of M. tuberculosis by droplet nuclei). (2) Preferential transmission is that which occurs when one route is the usual route but another route has been described; for example, transmission of the measles virus (rubeola) usually occurs by inhalation of droplet nuclei that are deposited in distal airways, but infectious droplets may also transmit the measles virus. (3) Opportunistic transmission is that which can occur when a pathogen usually transmitted by droplets can be transmitted by droplet nuclei (eg, influenza transmitted by aerosols of droplet nuclei) under unusual environmental conditions, such as intubation. Future studies may help define the applicability of this paradigm to CF pathogens.

\section{Summary}

In summary, CF pathogens can be transmitted by direct or indirect contact with infectious secretions, objects contaminated with infectious secretions, or infectious droplets. Recent data suggest that infectious droplets may travel as far as 6 feet (2 meters) from individuals with CF. While detection of infectious droplets is not confirmatory of patient-to-patient transmission, it is highly suggestive of the potential for such transmission. The potential for people with CF to generate droplet nuclei has been demonstrated in experimental models, but the relevance of these observations for transmission, including that of M. abscessus subsp. massiliense, has not been established and should be studied.

\section{Potential Sources of CF Pathogens}

\section{III.A. Person-to-Person Transmission among People with CF}

The source of CF pathogens is often unknown, and many individuals with $\mathrm{CF}$ are infected with unique strains of $P$. aeruginosa or Burkholderia spp. However, the molecular tools described above have expanded the evidence that people with $\mathrm{CF}$ can acquire CF pathogens from others with CF in both healthcare and nonhealthcare settings. These are the primary transmission and acquisition events targeted by the recommendations in this guideline. The relative frequency of shared strains of different CF pathogens is shown in Table $6 . .^{27,28,30,66,102,129-145}$

\section{III.A.1. Burkholderia spp.}

B. cenocepacia. Several methods have been used to genotype Burkholderia to define the epidemiology of infections in people with CF. $^{61,146-150}$ In the late 1980s, genotyping studies identified common strains in multiple individuals receiving care at the same CF centers, suggesting person-to-person spread. ${ }^{149}$ More compelling evidence soon followed. Transmission of B. cepacia complex was described at a CF educational retreat ${ }^{134}$ and among people with $\mathrm{CF}$ attending summer camps. ${ }^{151}$ Outbreaks were also reported within $\mathrm{CF}$ centers. ${ }^{135}$

Among so-called epidemic strains, the ET12 (electrophoretic type 12) strain was prevalent in eastern Canada and the United Kingdom. ${ }^{152,153}$ The Midwest strain and the PHDC (Philadelphia-Washington, DC) strain were identified in people with $\mathrm{CF}$ in the Midwest and mid-Atlantic regions of the United States, respectively. ${ }^{154-157}$ The ST04 strain (RAPD type 04) was identified in people with CF in western Canada, and the CZ1 strain (now referred to as ST32) was identified in 
most individuals infected with Burkholderia at the Prague CF center in the Czech Republic. ${ }^{158-160}$ Other B. cenocepacia strains have been shared among multiple individuals with $\mathrm{CF}$ in various Italian CF centers. ${ }^{161,162}$

Other species of B. cepacia complex. Shared strains from other species in the B. cepacia complex have been reported and generally involved smaller numbers of patients. ${ }^{133,161,163,164}$ A notable exception is B. dolosa strain SLC6, which was identified in an outbreak in a US CF center and associated with deterioration in lung function and increased mortality. ${ }^{25,133}$ The Glasgow strain of B. multivorans was identified in an outbreak among people with CF in the city in the early 1990s. ${ }^{133,165}$

However, the majority of Burkholderia-infected CF patients harbor genotypically distinct strains. $B$. multivorans and $B$. gladioli account for more than half of the Burkholderia infections in the United States, but it is uncommon that multiple individuals with CF share strains belonging to these 2 species. Thus, the majority of new Burkholderia infections in people with CF currently involve the acquisition of strains from independent sources, most likely the natural environment, as will be discussed further in Section III.D.1 below. ${ }^{16,167}$

Clinical impact of epidemic Burkholderia spp. Poor outcomes, including more rapid clinical decline, decline in lung function, and increased mortality both before and after lung transplantation ${ }^{165,168}$ have been associated with certain strains of B. cepacia complex. Outcomes from the Canadian and US CF patient registries demonstrated a 2.5 -fold increase in the relative risk of death in people with $\mathrm{CF}$ who are infected with B. cepacia complex. ${ }^{169,170}$ Overwhelming infection (the cepacia syndrome) has been reported with species of B. cepacia complex other than B. cenocepacia, including B. multivorans ${ }^{164}$ and $B$. dolosa SLC6. ${ }^{25}$ Both single-center and multicenter studies suggest that poor outcomes with $B$. cepacia complex may be related to species, and such strains as B. cenocepacia ET12 have been associated with the worst outcomes. ${ }^{171}$ Among 29 lung transplant recipients infected with $B$. cenocepacia $(n=$ $16), B$. multivorans $(n=11)$, and B. vietnamiensis $(n=2)$, all of the deaths occurred in those infected with $B$. cenocepacia. ${ }^{172}$

\section{III.A.2. P. aeruginosa}

Shared P. aeruginosa strains. Early strain-typing studies demonstrated that individuals with CF infected with P. aeruginosa harbored distinct strains, presumably acquired from the natural environment. ${ }^{61,173,174}$ Shared strains between siblings were well documented, ${ }^{175-177}$ and in 1986 a report from Denmark described the spread of a multidrug-resistant $P$. aeruginosa strain in a CF care center. ${ }^{178}$ In 1996, PFGE analysis of isolates recovered during an antibiotic trial identified a $\beta$ lactam-resistant strain of $P$. aeruginosa infecting 55 children at a CF center in Liverpool, United Kingdom. ${ }^{51}$ Other reports described shared or epidemic $P$. aeruginosa strains in the United Kingdom and Australia. ${ }^{53,179-181}$ PFGE analysis of 1,225
P. aeruginosa isolates recovered from people with CF receiving care in 31 treatment centers in the United Kingdom demonstrated that $28 \%$ of those infected harbored a strain shared with at least 1 other person with $\mathrm{CF}^{173}$ The 2 most prevalent strains accounted for more than $20 \%$ of the isolates examined. Some strains, including the Liverpool and Midlands 1 epidemic strains, were widely distributed and identified in $48 \%$ and $29 \%$ of CF treatment centers, respectively.

In Melbourne, Australia, a strain first detected in children with $\mathrm{CF}^{181}$ was subsequently identified in half of the individuals with CF who were infected with $P$. aeruginosa in Sydney. ${ }^{180}$ This strain, now referred to as the Australian epidemic strain 1 (AES-1), has also been identified in Brisbane. ${ }^{179}$ The Australian epidemic strain 2 (AES-2) is even more common in Brisbane, ${ }^{182}$ while the Australian epidemic strain 3 (AES3) is common in Tasmania. ${ }^{183}$ In Copenhagen, Denmark, PFGE and genomic DNA sequence analyses identified 2 major $P$. aeruginosa clones that have been common among and likely transmitted among people with CF for more than 2 decades. ${ }^{131}$ In the Netherlands, MLST analysis of $443 P$. aeruginosa isolates recovered from 265 individuals with CF in 2 CF centers identified 2 strains (designated ST406 and ST497) in 15\% and $5 \%$ of the patients. ${ }^{184}$ Furthermore, $60 \%$ of the individuals studied harbored a strain also found in at least 2 other individuals.

Strains common to large numbers of people with CF cared for in North America have also been described. In Vancouver, Canada, RAPD and PFGE were used to analyze $P$. aeruginosa isolates recovered between 1981 and 1999 from 174 individuals with CF; 157 distinct strains were identified, 123 of which were unique to individual patients. ${ }^{130}$ Several strains were shared by clusters of 2, 3, or 4 individuals, and 2 strains were shared by 21 and 18 individuals. More recently, in Ontario, Canada, MLST was used to analyze $P$. aeruginosa isolates recovered from 446 individuals with $\mathrm{CF}^{28}$ The LES was identified in $15 \%$ of these individuals, while a second strain (designated ST439) was found in 7\%. The route by which the LES was transmitted to people with CF in Canada is unknown.

In the United States, the presence of epidemic P. aeruginosa strains remains uncertain, as very few genotyping studies of isolates from large numbers of people with $\mathrm{CF}$ have been performed. In Houston, rep-PCR typing identified a multidrug-resistant $P$. aeruginosa strain in $32(45 \%)$ of 71 children with CF; this strain, designated Houston 1, appears to be distinct from other epidemic P. aeruginosa strains (J.J.L., written personal communication, October 2013). ${ }^{30}$ Compared with other strains, new infection with the Houston 1 strain was significantly more likely to occur in those children hospitalized within the 90 days prior to infection. In addition, compared with those infected with other strains, those with the Houston 1 strain spent 12 more days in the hospital in the year prior to acquisition. The authors found that following adoption of recommendations from the 2003 Infection Control Guideline for CF, transmission was halted. Furthermore, 
P. aeruginosa strains from participants in an antibiotic trial who were cared for at $18 \mathrm{CF}$ centers in the United States were evaluated with MLST; at each center, shared strains were noted in $0 \%-71 \%$ of participants, and 15 of 18 centers had participants with shared strains. ${ }^{132}$

Most epidemic strains of $P$. aeruginosa have had a multidrug-resistant phenotype, which facilitated their recognition. The presence of epidemic strains without an unusual or noteworthy phenotype might be difficult to detect. Active surveillance of sufficiently large numbers of isolates is required to monitor the presence and ongoing transmission of shared strains of $P$. aeruginosa. Such surveillance is not currently a component of routine CF care in the United States.

Routes of transmission and reservoirs of $\mathrm{P}$. aeruginosa. The epidemiologic and microbiologic basis for epidemic $P$. aeruginosa strains remains poorly understood, ${ }^{27}$ and it is unclear whether all epidemic $P$. aeruginosa strains have comparable capacity for patient-to-patient transmission. Strain differences in the production of infectious droplets or droplet nuclei that remain suspended in the air under experimental conditions may explain the differences in efficiency of transmission of epidemic P. aeruginosa strains. ${ }^{119}$

Infections with a shared strain ${ }^{130,173,179}$ are highly suggestive of patient-to-patient transmission, particularly as institution of IP\&C measures halted transmission. ${ }^{30,185}$ While acquisition from a common source is also a possibility, surveillance of inpatient and outpatient settings have not detected a reservoir for shared strains. ${ }^{51,53}$ Strains that are more widely distributed (ie, found in multiple CF care centers) might suggest contact among individuals with CF from different centers or, possibly, acquisition from the natural environment, as described below in Section III.D.

Clinical impact of epidemic Pseudomonas. The LES has developed increasing antibiotic resistance, ${ }^{186}$ and some epidemic strains of Pseudomonas are associated with clinical deterioration. ${ }^{185-188}$ The LES has been associated with an increased risk of death or lung transplantation during 3 years of follow-up and/or decline in lung function. ${ }^{28}$ Furthermore, those infected with the LES had a worse quality of life, including worse treatment burden, physical functioning, and respiratory symptoms, compared with those individuals infected with nonepidemic strains. ${ }^{189}$

\section{III.A.3. Other Gram-Negative Bacteria}

Several other nonfermenting gram-negative bacteria can cause intermittent or chronic infection in people with CF. Among these, S. maltophilia, Achromobacter spp., Ralstonia spp., Cupriavidus spp., and Pandoraea spp. are the most common. ${ }^{26,190}$

S. maltophilia. In the United States, 33 (80\%) of 41 individuals with CF infected with $S$. maltophilia harbored genetically distinct strains, and 4 clusters (each of 2 individuals) were detected, suggesting either patient-to-patient spread or acquisition from a common environmental source. ${ }^{140}$ Among
183 S. maltophilia isolates obtained from a multicenter antibiotic trial conducted in the United States, only 3 instances of shared strains were found. ${ }^{143}$ More recently, 110 isolates recovered from 50 individuals with $\mathrm{CF}$ were studied, and 5 distinct strains were identified that were each shared by 2 or 3 patients. ${ }^{141}$ Thus, while there is some evidence for shared strains of $S$. maltophilia and possible patient-to-patient transmission, most infections in people with CF appear to result from independent acquisition, most likely from non-healthcare-associated environmental sources or as a result of antimicrobial selective pressure.

A. xylosoxidans. A. xylosoxidans is an opportunistic pathogen that causes healthcare-associated infections, including bacteremia, meningitis, pneumonia, endocarditis, peritonitis, osteomyelitis, urinary tract infection, and endophthalmitis, in vulnerable hosts, including neonates, burn victims, and other immunocompromised patients. ${ }^{191-193}$

Among 341 individuals with CF in the United States infected with Achromobacter, $42 \%$ were infected with A. xylosoxidans, and $23.5 \%$ were infected with Achromobacter rhulandii. ${ }^{190}$ In 2 small single-center studies, Achromobacterinfected individuals each harbored genotypically distinct strains. ${ }^{194,195}$ However, there is some evidence that Achromobacter spp. are shared by people with CF. A CF center in the United States reported that $9(36 \%)$ of 25 Achromobacterinfected individuals harbored the same strain of A. xylosoxidans. ${ }^{142}$ In the same multicenter antibiotic trial described above, 92 Achromobacter isolates were analyzed, and 5 instances of shared strains ( 2 individuals each) were detected. ${ }^{143}$ In Athens, Greece, 5 of 9 individuals with CF infected with the same strain of A. xylosoxidans were close social contacts. ${ }^{196}$ In a multicenter study conducted in Belgium, 2 clusters of A. xylosoxidans strains were identified (one consisted of 4 individuals, and the other consisted of 10 individuals). ${ }^{144}$ Thus, there is some evidence of shared strains of A. xylosoxidans and a suggestion of possible patient-to-patient transmission.

Ralstonia, Cupriavidus, and Pandoraea spp. Several of the 15 species in the genus Ralstonia, including $R$. pickettii, $R$. mannitolilytica, and $R$. insidiosa, have been recovered from people with CF. ${ }^{197}$ In the United States, 25 (66\%) of 38 individuals infected with Ralstonia species had R. mannitolilytica; 9 had $R$. pickettii, 2 had $R$. gilardii, 1 had R. taiwanensis, and 1 had a Ralstonia species that could not be classified. ${ }^{198}$ Several of the 14 species included in the genus Cupriavidus, ${ }^{199}$ including C. pauculus, ${ }^{200}$ C. gilardii, ${ }^{201}$ C. respiraculi, ${ }^{202}$ and $C$. taiwanensis, ${ }^{203}$ have also been recovered from people with CF. ${ }^{199}$ Among isolates obtained in the United States from 2004 to 2008, Ralstonia and Cupriavidus species were recovered from 72 and $73 \mathrm{CF}$ patients, respectively. ${ }^{26} \mathrm{R}$. mannitolilytica accounted for $60 \%$ of Ralstonia species, while C. respiraculi was the most common (53\%) Cupriavidus species identified. Genotyping analyses of these isolates have not identified a strain common to more than 1 person with CF (J.J.L., unpublished data, October 2013). 
The genus Pandoraea, first described in 2000, is currently comprised of 9 species, all of which have been recovered from persons with $\mathrm{CF}^{204,205}$ Among the 74 individuals with $\mathrm{CF}$ in the United States with Pandoraea species recovered between 2004 and 2008, P. apista, P. pnomenusa, and P. sputorum accounted for approximately equal proportions of isolates. ${ }^{26} \mathrm{In}$ Denmark, a P. apista strain spread among 6 children with CF attending a winter camp, and most subsequently experienced a significant deterioration in lung function. ${ }^{206}$

\section{III.A.4. S. aureus}

Shared MSSA strains. S. aureus is normal skin flora and commonly colonizes the anterior nares. In 2003-2004, the anterior nares of approximately $35 \%$ of children in the United States without CF aged 1-19 years were colonized with S. aureus, ${ }^{207}$ and S. aureus can also be recovered from $48 \%$ of oropharyngeal swabs of healthy children 18 years of age and younger. ${ }^{208}$ In Germany, the anterior nares of 72 individuals with CF aged 1-25 years and 72 age-matched non-CF controls as well as 128 family members of 38 children with CF and 79 family members of 23 children without CF were studied to compare the frequency of $S$. aureus colonization. ${ }^{209}$ A significantly greater prevalence of nasal carriage of $S$. aureus $(66 \%)$ was found among those with CF who had not been treated with antistaphylococcal antibiotics during the 4 weeks preceding culture, compared with those recently treated $(29 \%)$ or those without CF (32\%). The proportion of family members colonized with $S$. aureus was similar among CF (32\%) and non-CF (35\%) families. PFGE analyses indicated that colonized individuals within the same family often shared the same $S$. aureus strain, indicating that $S$. aureus can be transmitted within families. The genome types found in people with CF and their families were also noted in the community.

Colonization does not usually have consequences, but it is a risk factor for subsequent disease in people with and without $\mathrm{CF}$; isolates colonizing the anterior nares and disease-producing isolates typically have the identical genotype..$^{209-212} \mathrm{In}$ people with CF, airway infection may be intermittent or chronic. ${ }^{91,213-216}$ Chronic infection of the airways with the same clone can persist for several years. ${ }^{91,209,213}$

Shared strains of S. aureus among individuals with CF are well documented. Four typing methods were used to compare MSSA strains recovered from individuals with CF before and after attendance at a 4 -week summer camp. ${ }^{136}$ Four of 20 patients acquired a strain noted in another camper at the start of camp, consistent with patient-to-patient transmission. In St. Louis, 2 episodes of transmission of MSSA between siblings with CF in which transmission to the younger siblings resulted in considerable morbidity have also been reported. ${ }^{137}$

Shared MRSA strains. In 2003-2004, the anterior nares of approximately $1.3 \%$ of children in the United States without CF aged 1-19 years were colonized with MRSA. ${ }^{207}$ In Australia, healthcare-associated transmission of MRSA among people with CF and the spread of MRSA from patients without CF to individuals with CF hospitalized in the same ward at the same time have been reported. ${ }^{138}$ In Leeds, United Kingdom, individuals with CF infected with MRSA spent more time in the hospital in the year prior to initial isolation of MRSA than age- and sex-matched uninfected control subjects with CF (19.8 vs 5.5 days; $P<.001){ }^{217}$

The molecular epidemiology of MRSA has also been studied to (1) understand the relative contribution of community versus traditional healthcare-associated clones, (2) compare the types of MRSA strains in CF versus non-CF individuals, and (3) assess the frequency of shared strains. In Dallas and Chicago, $88 \%$ of strains from children without CF were staphylococcal chromosomal cassette mec (SCCmec) type IV (socalled community-associated strains), while $65 \%$ of MRSA strains from children with CF were SCCmec type II (so-called traditional healthcare-associated strains); MRSA strains more recently acquired in children with $\mathrm{CF}$ were more likely to be SCCmec type IV. ${ }^{218}$ In addition, the distribution of MLST clonal complexes (CCs), which are closely associated with SCCmec types, was different among CF versus non-CF isolates. Among CF isolates, $71 \%$ were CC5, $26 \%$ were CC8, and $3 \%$ were $\mathrm{CC} 1$, whereas among non-CF isolates, $89 \%$ were CC8, $4 \%$ were CC5, and 7\% were CC1. While the reasons for differences in the molecular epidemiology of MRSA strains between non-CF versus CF individuals are unclear, the presence of common strains in the CF population suggest patient-to-patient transmission and/or potential virulence factors that facilitate colonization and infection in CF.

In Chapel Hill, North Carolina, similar findings were noted, as $72 \%$ of individuals with CF were infected with SCCmec type II strains, while only $17 \%$ were infected with SCCmec type IV strains. ${ }^{219}$ The latter group consisted of younger individuals. In a multicenter study conducted in the United States from 2008 to 2010, SCCmec type II strains were more common than SCCmec type IV strains, accounting for $71 \%$ of MRSA isolates, and $84 \%$ of SCCmec type IV strains that harbored Panton-Valentine leukocidin were USA300, the most common community-associated MRSA clone. ${ }^{220}$

In a multicenter study conducted in Italy, MLST analysis revealed common types in multiple individuals with CF attending multiple CF centers. ${ }^{139}$ Twenty-nine strains from 6 CF centers were identified as ST8 SCCmec type IV (USA), and 26 strains from $6 \mathrm{CF}$ centers were identified as ST5 SCCmec type I (also a healthcare-associated clone initially reported from the United Kingdom). Thus, epidemic lineages of MRSA from around the world have been identified in the CF population in Italy. It is not clear why the SCCmec type I and II strains predominate among individuals with $\mathrm{CF}$ at a time when SCCmec type IV predominates in non-CF populations. It is likely that more sensitive testing (eg, rep-PCR ${ }^{221}$ or whole-genome sequencing) will provide more precise information about the extent of person-to-person transmission of MRSA. ${ }^{222,223}$

Clinical impact of MRSA. Recent reports have demonstrated that chronic infection with MRSA is associated with 
increased morbidity and mortality. In a study using the CFFPR, when compared with 13,922 individuals with CF without chronic MRSA infection, 1,732 individuals chronically infected with MRSA had an increased rate of decline in lung function (decline in $\mathrm{FEV}_{1} \%$ predicted $1.44 \%$ per year vs $2.06 \%$ per year, respectively). ${ }^{22}$ Similar findings have been reported by others. ${ }^{224-226}$ In addition, MRSA has also been associated with increased mortality; in a cohort study of 19,833 individuals reported to the CFFPR with at least 2 years of follow-up, those with MRSA had a 1.27 higher risk of death when adjusted for severity of disease. ${ }^{23}$

\section{III.A.5. Nontuberculous Mycobacteria}

Shared NTM strains. Until recently, there was little evidence for shared strains of NTM species among individuals with $\mathrm{CF}^{102,227}$ In a multicenter study conducted in the United States, most of the 140 NTM isolates appeared to be distinct by single-locus ( $h s p 65$ ) sequence analysis. ${ }^{102}$ Among 14 NTMinfected individuals with CF included in a study in Sweden, a shared strain was found only in 1 pair of siblings. ${ }^{228}$

However, a recent report from the United States described the use of rep-PCR and PFGE typing to show the apparent spread of a strain of M. abscessus subsp. massiliense from a chronically infected adult with CF to 4 others attending the same clinic in Seattle. ${ }^{129}$ The index case and 2 others died within several months of becoming infected. Similarly, typing of 41 strains of $M$. abscessus complex from 17 individuals with $\mathrm{CF}$ in the United Kingdom using a novel variable-number tandem repeat scheme and an automated rep-PCR system found that most were persistently infected with a single clone, but some shared strains; no differences in clinical outcomes linked to specific strains was reported. ${ }^{145}$ In another recent report of NTM transmission, whole-genome sequencing and single-nucleotide polymorphism analysis were used to characterize $168 \mathrm{M}$. abscessus complex isolates from 31 individuals at a CF care center in the United Kingdom. ${ }^{66}$ Two clusters ( 1 consisting of 9 individuals and 1 consisting of 2 individuals) of M. abscessus subsp. massiliense were identified, with epidemiologic evidence of opportunities for person-to-person spread within the hospital setting. The strains from Seattle and the United Kingdom outbreaks are highly related and are also related to strains causing soft-tissue infections in Brazil. ${ }^{229}$ However, it is currently unknown why these strains from around the world are related. A third outbreak of $M$. abscessus occurred from 2009 to 2011 at a pediatric CF center in Hawaii, in which 9 (55\%) of 17 children were infected with the same strain as identified by PFGE. ${ }^{230}$ An investigation conducted in conjunction with the Department of Health revealed that the PFT laboratory was the most likely source of transmission, as the infected patients performed PFTs at the same time in very close proximity.

Clinical impact of NTM. M. abscessus in particular is challenging to treat and may be associated with rapid clinical deterioration and poor outcomes after lung transplanta- tion. ${ }^{231-233}$ A multicenter prospective study conducted more than a decade ago showed no association between M. abscessus and decline in lung function, ${ }^{102}$ but a longer single-center study showed that chronic M. abscessus infection was associated with an excess decline of $0.78 \%$ predicted $\mathrm{FEV}_{1}$ per year. $^{66,234}$

\section{Summary}

In summary, epidemiologic studies have shown that individuals with CF can share the same strain of several CF pathogens. For decades, person-to-person transmission of Burkholderia spp. has been described, while person-to-person transmission of $P$. aeruginosa has been increasingly recognized in CF centers worldwide. MRSA strains detected in people with CF have been identified as epidemic clones, causing both healthcare- and community-associated infections in both CF and non-CF populations. Thus, studies of transmission of MSSA and MRSA are confounded by the fact that such species commonly colonize and infect people without CF. Most recently, compelling evidence of person-to-person transmission of $M$. abscessus has been described. Currently, the route(s) of transmission, including the potential for transmission by droplet nuclei, and the role played by mycobacterial virulence factors are under investigation. Multicenter studies, core laboratories, and use of advanced molecular methodologies are needed to understand the frequency and routes of transmission of mycobacteria in persons with CF. Epidemic strains of Burkholderia, Pseudomonas, and M. abscessus as well as infection with MRSA have been associated with increased morbidity and mortality. Use of higher resolution typing, such as whole-genome sequencing, is needed in future studies to provide a more precise understanding of the dynamics of transmission.

\section{III.B. Acquisition from People without $C F$}

\section{III.B.1. P. aeruginosa and Burkholderia spp.}

Approximately $10 \%$ of people without CF may have gastrointestinal tract colonization with $P$. aeruginosa. P. aeruginosa is a well-described opportunistic pathogen of immunocompromised non-CF individuals, including oncology patients, burn victims, and ventilated patients. ${ }^{235-237}$ To our knowledge, there is only 1 case report of transmission of P. aeruginosa to the non-CF household members of a person with CF. Both parents of a 22-year-old woman with CF, infected with the LES, developed pneumonia caused by this strain. ${ }^{238}$ Both were carriers of abnormal CFTR, but neither had CF.

B. cepacia complex strains do not colonize people without $\mathrm{CF}^{239}$ but can cause infections in individuals who are immunocompromised, including those with chronic granulomatous disease or solid-organ transplantation. ${ }^{240,241}$ To our knowledge, there is only 1 case report describing hospital transmission of Burkholderia from a non-CF individual with 
chronic respiratory failure to multiple patients with and without $\mathrm{CF}^{242}$

\section{III.B.2. S. aureus}

Unlike other CF pathogens, both MSSA and MRSA can colonize and infect non-CF individuals, including household members and other close contacts. Thus, there is the potential for acquisition of $S$. aureus in healthcare and community settings from people without CF. Furthermore, as described above, healthcare- and community-associated MRSA strains can infect people with $\mathrm{CF}^{218,220}$ Definitive evidence of transmission of MRSA, particularly community-associated strains, may be confounded by the observation that relatively few clones have been described, and highly sensitive molecular techniques have been used only in recent years.

MRSA colonization and infections in non-CF patients. For decades, MRSA infection and colonization in patients without CF were exclusively associated with hospitalization, chronic care facilities, or dialysis units. ${ }^{243}$ Beginning in the late 1990s, MRSA infections began to occur in the community setting in previously healthy individuals, so-called community-onset or community-acquired MRSA (CA-MRSA). ${ }^{244}$ Skin and soft-tissue infections are the most common manifestation of CA-MRSA, but necrotizing pneumonia, bone infections, and sepsis are also described. ${ }^{245-248} \mathrm{~A}$ recent metaanalysis assessing MRSA colonization of the anterior nares in children described a prevalence of $5.4 \%$ in hospitalized children and 3\% in children in the community, suggesting that MRSA colonization is relatively common in the general population..$^{249}$

Transmission from non-CF patients. To our knowledge, there is only 1 report describing healthcare-associated transmission of MRSA from patients without CF to patients with CF. In Australia, transmission of MRSA from patients without $\mathrm{CF}$ to individuals with $\mathrm{CF}$ hospitalized in the same ward at the same time was described. ${ }^{138}$ However, the authors did not describe the routes of transmission and the IP\&C strategies that were in place.

CF households. In a multicenter study of children with CF with MRSA in New York, non-CF household members had anterior nares colonization with the same strain of MRSA ( $14.7 \%$ vs $1.6 \%$ of case vs control household members). ${ }^{215}$ While the direction of transmission is unknown, it is likely that the non-CF family member acquired MRSA from his or her child with CF. No staphylococcal infections occurred in household members.

Healthcare personnel. The anterior nares of healthcare personnel without CF may be colonized with MRSA. However, routine screening is not recommended, unless healthcare personnel are epidemiologically associated with ongoing transmission..$^{20,250-252}$ In contrast, healthcare personnel without CF who develop symptomatic MRSA infections (eg, draining wounds, sinusitis with drainage, and superinfection of chronic dermatitis) should be placed on administrative leave, treated until no longer infectious, and obtain clearance from the facility's occupational health service before returning to work. ${ }^{5,8}$

Other nonhealthcare settings, including sports teams. Participation in sports has been identified as a risk factor for the development of MRSA colonization and infection in the non-CF community, presumably due to skin colonization, skin abrasions, intimate contact between players, and poor hygiene. ${ }^{253,254}$ MRSA has been detected both in the environment (eg, locker room, strength and conditioning equipment, and whirlpool equipment) and in samples obtained from athletes (eg, nose, skin sites, and shoes). ${ }^{254-258}$ In addition, outbreaks of MRSA skin and soft-tissue infections have been reported among members of sports teams..$^{255,259-267}$ However, to our knowledge there are no reports of transmission of MRSA from a person with CF on a sports team to another athlete without CF or vice versa. Thus, there are no data to support exclusion of an individual with CF with MRSA in their respiratory tract from participation in sports.

The CDC has developed recommendations to prevent MRSA transmission among athletes and in athletic facilities. ${ }^{268}$ They include the following: (1) improve hygiene among athletes by covering and containing wounds, showering after participating in sports activity, washing and drying uniforms after each use, not sharing personal items (eg, razors), and reporting possible infections to the team physician, athletic trainer, school nurse, or primary care doctor so that treatment can be initiated promptly; (2) clean and disinfect athletic facilities, including showers, using appropriate cleaning and disinfection measures; and (3) exclude athletes with MRSA wound infections from participation if wounds cannot be completely covered or if a healthcare provider determines that the infection poses a risk to the individual with the infection. Athletes with MRSA infections should not use common-use water facilities or pools until the infection has resolved.

\section{III.B.3. Respiratory Viruses}

Viral respiratory pathogens, including respiratory syncytial virus (RSV), rhinovirus, and influenza virus, pose a risk to people with CF. In adults and children with CF, viral infections can trigger pulmonary exacerbations, ${ }^{269-272}$ particularly influenza virus ${ }^{271,273}$ and rhinovirus. ${ }^{269,270}$ Exacerbations in which a virus is identified are associated with worse clinical severity compared with viral exacerbations. ${ }^{274}$ As sensitive viral detection methods like real-time PCR become increasingly available and identify a broader range of viruses, ${ }^{275}$ the role played by other viral pathogens (eg, coronavirus and human metapneumovirus) in CF exacerbations will be further elucidated.

\section{Summary}

Transmission of gram-negative bacterial pathogens between people with $\mathrm{CF}$ and people without $\mathrm{CF}$ is very rare. Given 
the recent observations that people with $\mathrm{CF}$ are infected with community-associated MRSA, it is feasible that some transmission of $S$. aureus is occurring between people with and without CF. Further studies are needed to assess the extent to which this occurs. In contrast, it is highly likely that viral pathogens are frequently transmitted between people with and without $\mathrm{CF}$, given the annual community outbreaks of seasonal viruses and the high transmissibility of these agents.

\section{III.C. Acquisition from Animals}

Overview of zoonotic infection. More than 800 microorganisms can cause zoonotic infections, defined as pathogens transmitted from animals to humans or from humans to animals. The CDC's National Center for Emerging and Zoonotic Infectious Diseases is charged with preventing disease and disabilities from such infections. ${ }^{276}$ People with CF can have opportunities for close contact with animals, including with personal pets, service animals, and pet therapy animals as well as farm animals and animals in petting zoos.

Pets. A few reports have addressed transmission of pathogens from animals to people with CF and from people with CF to animals. ${ }^{277-281}$ Following lung transplantation, Bordetella bronchiseptica pneumonia developed in 2 children with CF who acquired this pathogen from ill pet dogs, and 1 child died. ${ }^{278-280}$ B. bronchiseptica was also detected in the respiratory tract of a child with $\mathrm{CF}$ who acquired this organism from an ill kitten, and this child had no adverse effects following treatment. ${ }^{280}$ The LES of $P$. aeruginosa was transmitted from a 54-year-old man with CF to his pet cat; the cat developed respiratory symptoms. ${ }^{281}$ The reptile collection of a person with $\mathrm{CF}$ was cultured and not found to harbor potential CF pathogens. ${ }^{277}$

MRSA colonization and infections have been described in dogs, cats, rabbits, hamsters, guinea pigs, turtles, chinchilla, and birds. ${ }^{28,283}$ Outbreaks of MRSA have been described in animal hospitals, and both hospital- and community-associated strains have been identified. Most of these are sporadic infections, but when outbreaks occurred, they are thought to reflect initial transmission from people to animals. ${ }^{282,283}$ There are limited data on the frequency of MRSA transmission from animals to humans, and there are no published data describing this phenomenon in people with CF.

Mycobacterium marinum skin and soft-tissue infections have been linked to cutaneous exposure to fish tanks. ${ }^{284} \mathrm{In}$ fections have occurred in both immunocompetent and immunocompromised patients, including transplant recipients. ${ }^{285}$ Risk factors included exposure to fish tanks, the presence of open skin lesions during the cleaning of fish tanks, fishing, and exposure to aquarium water. There are no published reports of M. marinum infections in individuals with CF linked to fish tank exposure.

The CDC has several IP\&C recommendations for people who own pets that are relevant for people with CF who own pets. ${ }^{286}$ These recommendations emphasize using hygienic principles to prevent zoonotic infections, including (1) hand hygiene, (2) disinfecting cages or tanks, (3) appropriate preventive care for pets, (4) prompt assessment of ill pets by a veterinarian, and (5) wearing gloves while cleaning fish tanks. Handling of reptiles is not recommended for any individuals at risk of serious complications of Salmonella infections.

Pet therapy and service animals. Many institutions, particularly children's hospitals, have introduced pet therapy programs that allow pets that have been certified to be free of certain infections to visit patients in healthcare settings. Recommendations for such programs include (1) training programs for the dogs and their owners, (2) stringent criteria for animal vaccinations and cleanliness, and (3) criteria for eligible patients. ${ }^{287,288}$ The importance of hand hygiene before and after contact with the animals is emphasized. Patients under Transmission-Based Precautions, those with animal allergies, or children frightened by animals are generally excluded from participation. To date, there are no reports of transmission of potential pathogens from pet therapy animals to people with CF. People with CF can participate in such programs as per local institutional policies.

Service animals represent unique interactions between people and animals by providing guidance and support for both physical and emotional disabilities. There are no published reports of transmission of potential pathogens from service animals to people with CF. People with CF can participate in such programs when instituted as per relevant state and federal guidelines. ${ }^{289}$

Farm animals. Farm animals have been associated with several types of zoonotic infections. Farm animals have been linked to transmission of viral pathogens to humans, most notably influenza virus from pigs (http://www.who.int /topics/influenza/en/) and coronavirus. ${ }^{290}$ Animal stalls, sheds, and coops may become heavily contaminated with fecal flora as well as with Aspergillus and other molds that proliferate in hay and other organic matter. Thus, it would be prudent for people with CF to avoid cleaning stalls, pens, or coops and to perform other chores instead.

To date, there are no reports of transmission of potential pathogens from farm animals to people with CF. Nonetheless, farm animals may represent a source of potential pathogens for people with CF. Theoretical concerns include (1) influenza infections; (2) ABPA caused by cleaning stables; (3) MRSA from horses and pigs; and (4) P. aeruginosa from horses. ${ }^{282,291}$

In summary, while there are limited data describing zoonotic infections in people with CF, people with CF and their families should follow preventive strategies described in national guidance documents.

\section{III.D. Acquisition from the Inanimate Environment}

\section{III.D.1. Nonhealthcare Sources: Soil, Organic Matter, and Water}

Overview. There has been continuing concern that the natural environment (eg, water and soil) may be a reservoir for CF pathogens, such as P. aeruginosa, Burkholderia spp., NTM, 
and Aspergillus spp. P. aeruginosa is ubiquitous in rivers, aquatic areas, soil, and plants worldwide. B. cepacia complex can be found in rice, wheat, and maize rhizospheres and in human sewage, and B. gladioli and B. cepacia are well-recognized plant pathogens. Other species, particularly Burkholderia ambifaria, exist within the rhizospheres of certain plants, while B. multivorans is infrequently recovered from the environment. ${ }^{292-296}$ Several investigations since the publication of the 2003 Infection Control Guideline for CF have enhanced our understanding of the potential role played by the natural environment in the acquisition of CF pathogens and possible strain-specific reservoirs.

Burkholderia $\boldsymbol{s p p}$. Implementation of strict IP\&C measures has eliminated new acquisition of several epidemic Burkholderia strains, including ET12 and SLC6, among people with CF. ${ }^{154,297}$ The incidence of infection with the PHDC and the Midwest clone has decreased but has not been eliminated with improved IP\&C practices, and individuals with CF have been infected in the absence of apparent contact with others with CF (J.J.L., unpublished observations, October 2013). Strain PHDC has been recovered from agricultural soil in the United States, including onion fields, and from people with CF in diverse locations in the United States and Europe, suggesting that this strain is widely distributed in the natural environment, which serves as a source of ongoing acquisition. ${ }^{161,298-300}$ Strains identical to those found in people with CF have also been isolated from onions and onion rot. ${ }^{301,302}$ Furthermore, MLST analysis demonstrated that more than $20 \%$ of $381 \mathrm{CF}$ isolates of Burkholderia were indistinguishable from strains recovered from the natural environment. ${ }^{301} B$. cepacia complex isolates with the same nucleotide identity at all 7 MLST loci have been isolated from people with CF and from river water, suggesting another possible environmental reservoir. ${ }^{302}$ Finally, B. cenocepacia and Burkholderia vietnamiensis have been isolated from human sewage in the United Kingdom, but genotyping of these isolates was not performed. ${ }^{303}$ In contrast, the Midwest strain of B. cenocepacia has not been found in the natural environment in regions where this strain infects people with CF. ${ }^{157,295}$

P. aeruginosa. In the homes of people without $\mathrm{CF}, P$. aeruginosa was detected most often from kitchen and bathroom drains but not from soil. ${ }^{304}$ Similarly, in the homes of people with CF, $P$. aeruginosa was detected most often from shower drains and bathroom drainpipes. ${ }^{305,306}$ While $P$. aeruginosa was recovered from $34 \%$ of the homes of newly infected people with CF ( $6 \%$ of samples), only 9 (18\%) of 50 paired environmental and patient isolates were the same strain. ${ }^{306}$ It remains unclear whether these $P$. aeruginosa strains were transmitted from the individuals with CF to the home environment or vice versa, but it suggests that cleaning and disinfection efforts in the homes of people with CF should focus on bathroom drains. There are insufficient data to determine the optimal frequency of cleaning showerheads. However, showerheads that have smoother surfaces may be less prone toward retaining organisms than those that have more crevices.
In Switzerland, a very low prevalence of $P$. aeruginosa was detected in public outdoor pools, standing water, and running water from the bathroom taps of people with $\mathrm{CF}^{307}$ P. aeruginosa has been isolated from whirlpool spas and hot tubs, and outbreaks of folliculitis and more serious infections caused by $P$. aeruginosa have been associated with hot tubs and whirlpool bathtubs. ${ }^{1}$ In the United States, an observational study of children with newly acquired $P$. aeruginosa did not find that hot tub use was associated with age at $P$. aeruginosa acquisition but did find that swimming pool use in the previous year was protective. ${ }^{308}$ These findings may reflect the relatively healthy pulmonary status of the children with CF enrolled in this study.

Natural bodies of fresh water (eg, rivers and lakes) have not been definitely proven to be a source of CF pathogens, but stagnant water should always be avoided due to a heavy burden of potential pathogens that flourish in organic debris. However, after heavy rains fresh bodies of water may be contaminated with sewage overflow, and local/state monitoring should be reviewed before swimming in such water.

In Germany, the $P$. aeruginosa strain designated clone $\mathrm{C}$ has been recovered from individuals with $\mathrm{CF}$ and from environmental samples from geographically diverse areas. ${ }^{309}$ This same strain was subsequently identified from people with $\mathrm{CF}$ in the United Kingdom, ${ }^{173}$ further suggesting a broad distribution of this particular strain in the natural environment. Other $P$. aeruginosa epidemic strains have not been identified in environmental samples, although genotyping surveys of large numbers of strains recovered from the environment have not been performed.

Other gram-negative bacteria. S. maltophilia is commonly found in soil and has been identified in well and river water, stream sediment, raw milk, frozen fruit, and sewage. ${ }^{310,311}$ Species other than A. xylosoxidans, A. rhulandii, Achromobacter piechaudii, and Achromobacter denitrifican are found in soil and rarely cause human infections. ${ }^{312}$

NTM. The natural habitat for NTM is soil and water, ${ }^{313}$ and the prevalence of NTM in the natural environment has wide geographical variation. Under experimental conditions, high numbers of pathogenic NTM were recovered from aerosols produced by 2 commercial potting soils. When NTMinfected individuals without CF submitted their own potting soils for PFGE analysis, 1 patient-soil pair had indistinguishable strains of $M$. avium and 2 patient-soil pairs had closely related strains of $M$. intracellulare, suggesting that potting soil could be a reservoir for NTM for people with CF who have intense, repeated exposures. ${ }^{314}$ Another study isolated $M$. avium-intracellulare from $49 \%$ of residential soil samples in Japan and found 6 clinical and corresponding soil isolate pairs with identical genotypes from non-CF case patients with high soil exposure, defined as more than or equal to 2 hours/week, including digging or carrying soils, mowing grass, planting flowers, and exposure to soil dusts when farming or gardening. ${ }^{315}$ Thus, although these studies were not conducted in people with $\mathrm{CF}$, they do suggest that it may be prudent to limit exposure to soil. 
Aspergillus spp. and other filamentous fungi. The 3 most frequent species of filamentous fungi isolated from people with CF are A. fumigatus, Scedosporium apiosporium, and A. terreus. The natural habitat for Aspergillus spp. is soil, where they function as saprophytes growing on organic debris and recycling carbon and nitrogen throughout the environment. As a result, aerosolized conidia are ubiquitous in the environment. Characteristics of A. fumigatus that promote successful colonization of the airways include thermotolerance, small and abundant conidia, fast growth rates, and production of toxic metabolites and enzymes that are effective in breaking down complex polysaccharides. A. terreus is seldom reported from environmental sources, but in one study of air and surface samples from patient rooms and soil from the park adjacent to the hospital, A. terreus was found only in the soil samples; however, there were no common genotypes in patient and soil samples. ${ }^{105} \mathrm{~S}$. apiosporium is found in highly polluted soils and water but is rarely encountered in the environment. In one study of air and surfaces in the homes of 6 people with CF, large concentrations of S. apiosporium were isolated from $36(65 \%)$ of 55 potted plants, but no genotyping was reported. In conclusion, the intensity and duration of exposure to these environmental reservoirs of filamentous fungi may increase the risk of acquisition by people with CF. However, without genotyping studies, this link remains unconfirmed.

Summary. Although many CF pathogens may be found in the inanimate environment, there are few instances where the same genotypes are found in the natural environment and in isolates from the respiratory secretions of people with CF. Nonetheless, it is prudent to avoid activities that include prolonged and intense exposure to soil, construction, and swimming in pools that are not appropriately chlorinated or swimming in stagnant water.

\section{III.D.2. Healthcare Sources: Water, Surfaces, Equipment,} Air, and Contaminated Products

Water and other fluids. Water has been the source of healthcare-associated infections and linked to outbreaks caused by Legionella, Pseudomonas, Stenotrophomonas, Burkholderia, and Achromobacter. ${ }^{316}$ In both inpatient and ambulatory healthcare settings where people with CF receive care, the highest number of positive environmental cultures for $P$. aeruginosa were from the sink drains or showers in patient rooms. ${ }^{127,317}$ One study found genetic relatedness in 19 of 21 clinical and environmental strains, but it was uncertain whether $P$. aeruginosa was transmitted from the individuals with $\mathrm{CF}$ to the clinical environment or vice versa. ${ }^{317}$

The sources of healthcare-associated infections caused by S. maltophilia are poorly understood. In non-CF patients, $S$. maltophilia has been linked to inappropriate use of hand moisturizing lotion, rather than soap, by healthcare person$\mathrm{nel}^{318}$ and to contamination of faucet aerators in intensive care unit (ICU) sinks. ${ }^{319}$ Furthermore, S. maltophilia has been recovered from hospital sink drains, faucets, and potable water. ${ }^{140,320}$ In general, genotyping analyses have not shown that isolates obtained from the hospital environmental are the same as those recovered from patients, ${ }^{141,320}$ and the majority of non-CF patients infected with S. maltophilia had genetically distinct strains. ${ }^{321}$ None of 24 S. maltophilia isolates recovered from water, taps, and sinks in patient rooms matched the strains recovered from individuals with CF.

Despite these occasional outbreaks related to a water source, routine environmental sampling, including culturing water supplies, is not advised, except for water-quality determinations in hemodialysis settings and other situations where sampling is directed by epidemiologic principles, and results can be applied directly to infection control decisions. ${ }^{2}$ Removal, cleaning, and disinfecting of showerheads and tap aerators once a month with an EPA-registered product or a chlorine bleach solution (500-615 ppm [1:100 dilution]) has been recommended as part of Legionella control measures, but there are insufficient data to support a widespread recommendation and specific time intervals, given the infrequency of outbreaks associated with faucet aerators. ${ }^{2}$

Healthcare surfaces. Several studies in healthcare facilities, many of which utilized molecular typing methods, have identified the same pathogens on inanimate surfaces as those recovered from non-CF patients, including VRE, MRSA, $C$. difficile, Acinetobacter spp., and norovirus. ${ }^{322-325}$ Pathogens shed by patients can contaminate healthcare surfaces at concentrations sufficient for transmission, can survive for extended periods of time, and can be transferred to the hands and clothing of healthcare personnel, leading to further transmission. ${ }^{5}$ Surface contamination with VRE resulted from the failure to clean rather than faulty cleaning methods or products, thereby supporting the concept that education about cleaning and compliance monitoring could reduce environmental contamination. ${ }^{326}$ As further evidence of the role played by contaminated surfaces in hospital-associated infections, a patient admitted to a room previously occupied by a patient colonized or infected with a pathogen (eg, MRSA, VRE, C. difficile, or Acinetobacter spp.) has an increased likelihood of developing colonization or infection with that pathogen due to inadequate decontamination of surfaces. ${ }^{5,327-329}$

In healthcare facilities that deliver care to people with CF, contamination of dry environmental surfaces with CF pathogens was low, but contamination of the hands of people with CF was higher. ${ }^{128}$ In a Liverpool CF center, contamination of inanimate surfaces was transient and negative after patient discharge and routine cleaning. ${ }^{127}$ Similar findings have been noted in other CF clinics. ${ }^{128,317}$

Healthcare equipment. Contamination of medical equipment is another potential source of pathogens for people with CF. During simulated examinations, stethoscopes acquired and transferred MRSA and C. difficile nearly as often as gloved hands, ${ }^{330}$ and RSV has been detected on stethoscopes in nonCF settings. ${ }^{331}$ In CF settings, 26 stethoscopes used in a clinic were not found to be contaminated with CF pathogens, ${ }^{332}$ and 
in a more recent study $P$. aeruginosa and $S$. aureus were rarely recovered from stethoscopes, pulse oximeters, and otoscopes. ${ }^{128}$ Nevertheless, there is sufficient evidence to support the importance of cleaning and disinfecting medical equipment after use by one person with CF prior to use by another person with CF, according to hospital protocols.

Numerous outbreaks (and pseudo-outbreaks, ie, no evidence of infection or disease linked to recovery of microorganism) of $P$. aeruginosa, other gram-negative bacilli, and NTM have been linked to contaminated medical devices. For example, outbreaks of $P$. aeruginosa have been linked to inadequate processing of rigid $^{333,334}$ and flexible laryngoscopes, ${ }^{333}$ flexible bronchoscopes, ${ }^{335-337}$ and defective reprocessors for bronchoscopes and endoscopes. ${ }^{338}$ Similarly, outbreaks and pseudo-outbreaks of NTM have been linked to bronchoscopes, ${ }^{339}$ and a hospital hydrotherapy pool contaminated with $M$. chelonei that led to infections in children with $\mathrm{CF}^{340}$

Air. Infectious droplets in the air may represent another source of transmission for CF pathogens. P. aeruginosa was recovered from the air in hospital rooms 45 minutes to 2 hours after people with CF left, ${ }^{126,127}$ and the LES of $P$. aeruginosa was recovered from the corridors of CF clinics as long as 3 hours after individuals had left the area. ${ }^{127}$ In the United Kingdom, after individuals with CF performed PFTs or nebulization, the Manchester epidemic strain of $P$. aeruginosa as well as nonepidemic $P$. aeruginosa were recovered from the air. $^{341}$ In France, the concentration of $P$. aeruginosa was highest after the person with CF awoke or performed chest physiotherapy. ${ }^{126}$ Several CF pathogens (ie, P. aeruginosa, MSSA, and MRSA) were recovered from air collected 3 feet from CF subjects in exam rooms; these strains were the same as those infecting the subjects as assessed by PFGE. ${ }^{128}$ Similarly, CF pathogens were recovered from the air 6 feet from CF subjects performing PFTs. ${ }^{125}$ Factors associated with generating infectious droplets of $P$. aeruginosa are unknown, as exacerbations, sick versus well CF clinic visits, or age were not predictive. ${ }^{125,128}$ We emphasize that none of these observational studies were associated with person-to-person transmission, but such data provide evidence of the potential for such transmission and, thus, the importance of implementing strategies to prevent droplet transmission. Furthermore, as previously described the potential for transmission of $M$. abscessus by droplets or droplet nuclei has been suggested, ${ }^{66,129}$ but to our knowledge no studies to date have demonstrated the specific route(s) of NTM transmission among people with CF.

Contaminated products. Burkholderia spp. are the most frequently isolated bacteria in nonsterile and sterile pharmaceutical products that have been recalled. ${ }^{342,343}$ Many healthcareassociated outbreaks of Burkholderia infections associated with contaminated skin antiseptics, mouthwashes, ultrasound gels, medications, and medical devices have been described. ${ }^{344-362}$ Other products that have become contaminated during use include nasal irrigation bottles contaminated with $P$. aeruginosa, ${ }^{363,364}$ multiuse albuterol vials contaminated with $B$. cepacia, ${ }^{348,357}$ acupuncture devices disinfected with glutaraldehyde contaminated with $M$. abscessus, ${ }^{365}$ cosmetic surgical supplies contaminated with mycobacterial spp., and supplies used for liposuction. ${ }^{366}$ Outbreaks of Achromobacter spp. have been attributed to contaminated disinfectant solutions, dialysis fluids, saline solution, and deionized water. ${ }^{367}$ In addition, products used in nonhealthcare settings can become contaminated during manufacturing and can cause extensive outbreaks. These have included alcohol-tattoo ink contaminated with $M$. chelonae $^{368}$ and footbaths in nail salons contaminated with NTM spp. ${ }^{369}$

Summary. Most potential sources of pathogens that have been identified in the healthcare environment can be eliminated by following facility processes for the cleaning and disinfection of surfaces and equipment. Although there are no published reports of proven acquisition by people with CF from contaminated products, such sources present a potential risk and should be considered during any outbreak investigation in people with CF. The CF care team should receive alerts for contaminated products sent by the Food and Drug Administration's (FDA's) MedWatch and the CDC's Health Alert Network.

\section{III.D.3. Construction and Renovation}

Multiple outbreaks of airborne filamentous fungi, principally due to Aspergillus spp., have been reported in hospitals associated with construction, renovation, repair, and demolition. ${ }^{370-372}$ While most of the outbreaks were related to construction or renovation, problems with the air supply system have also been implicated. The most common species involved in the outbreaks were A. fumigatus and A. flavus, although a substantial number involved more than 1 species of Aspergillus. Groups at highest risk for nosocomial infection during these outbreaks included persons with hematologic malignancy, solid-organ transplantation, and other immunocompromising conditions (eg, high-dose steroid therapy).

We were unable to find any studies assessing a healthcare source for Aspergillus in CF. Furthermore, CF has not been described as a risk factor for acquisition of invasive aspergillosis in nosocomial outbreaks related to construction, renovation, repair, or demolition. Nevertheless, it is prudent to ensure that dust-containment strategies are followed throughout the entire healthcare facility.

\section{Strategies to Reduce Transmission and Acquisition of CF Pathogens}

\section{IV.A. Overview}

Several published experiences have described the effectiveness of stringent IP\&C practices and policies for reducing the prevalence of epidemic $B$. cepacia complex, ${ }^{25}$ the LES strain, ${ }^{1,373,374}$ and other transmissible strains of $P$. aeruginosa. ${ }^{30,185,375,376}$ Interventions have included education of healthcare personnel and people with CF and their families about risk factors for 
routes of transmission and preventive strategies, emphasizing hand hygiene for people with CF and healthcare personnel, the use of single-patient rooms when people with CF are admitted to the hospital, Contact Precautions, avoiding socializing in both healthcare and nonhealthcare settings, improving detection of CF pathogens in microbiology laboratories, decontaminating the healthcare environment, and cohort segregation of patients known to be harboring specific pathogens while in a CF clinic. ${ }^{1}$ Most recently, efforts to prevent further transmission of M. abscessus subsp. massiliense in an adult $\mathrm{CF}$ clinic included educating and reinforcing IP\&C strategies for $\mathrm{CF}$ to the $\mathrm{CF}$ care team and individuals with $\mathrm{CF}$, cleaning all clinic and equipment surfaces twice, and the use of a negative pressure room for all NTM-infected individuals. ${ }^{66,129}$ In a pediatric CF center experiencing an outbreak of $M$. abscessus, investigators concluded that the PFT laboratory was the most likely source of transmission and changed from performing PFTs in a central laboratory to performing portable spirometry in clinic exam rooms. ${ }^{230}$ Notably, several strategies were used to control each outbreak and most likely included enforcing preexisting IP\&C practices. Thus, it is impossible to conclude which intervention(s) were most effective. However, similar to other prevention programs in IP\&C - such as reducing device-related infections, including central line-associated bloodstream infections and ventilatorassociated pneumonia-it is likely that implementation of a bundle of practices is required to reduce transmission of $\mathrm{CF}$ pathogens. ${ }^{377-379}$

The following sections describe the IP\&C strategies recommended in this updated guideline. The recommendations are intended for all people with CF, regardless of respiratory tract culture results. The recommendations should also be followed for people with CF following lung or liver transplantation, as such individuals are immunocompromised, at risk of becoming infected from others with CF, and following transplantation may continue to harbor $\mathrm{CF}$ pathogens. The recommendations for healthcare settings are intended for CF clinics and other ambulatory care areas, inpatient settings, diagnostic areas, and all clinical research activities. Successful and consistent implementation of IP\&C practices must include the ongoing participation of people with $\mathrm{CF}$ and their families and auditing the performance of healthcare personnel. Depending on available resources, a center may choose to implement the recommended IP\&C practices in all areas at once or may choose to stage the implementation by setting.

\section{IV.B. Education Strategies}

Overview. To successfully implement the recommendations in this guideline, several stakeholders must be educated, including (1) all healthcare personnel who have contact with people with CF (eg, physicians, nurses, respiratory and physical therapists, radiology and laboratory personnel, social workers, operating room staff members, research coordina- tors, administrative personnel, and environmental services personnel); (2) all people with CF, from toddlers to adults (individuals may have varied age-appropriate experience, knowledge, and motivation regarding IP\&C); and (3) families and friends of people with CF.

Families of older adolescents and adults with CF may struggle to accept the paradigm shift that has taken place for IP\&C during the past decade on the basis of new knowledge of transmission of CF pathogens. Families of newly diagnosed individuals are more likely to be receptive to the current recommendations, as they have less prior experience. Therefore, different strategies may be required for educating different groups.

Educators must recognize the needs and levels of understanding of various groups of stakeholders to optimize the effectiveness of educational programs. While the CF Foundation has developed educational tools, individual CF center staff may also create their own tools and include people with $\mathrm{CF}$ and their families in the development process. Fortunately, the disciplines of CF and of IP\&C have a great deal of experience providing education to other healthcare personnel, patients, and families. Some examples include the myriad educational materials to promote proper hand hygiene, cough etiquette, and respiratory hygiene aimed at preventing the spread of pathogens transmitted by the droplet route; these are available in many languages and literacy levels. ${ }^{380}$ Information is also available on the CF Foundation's website (http://www .cff.org).

Education should be provided to people with CF and their families on a regular basis using a variety of learning methods, including written, visual, demonstration, and return demonstration. In a survey of people with $\mathrm{CF}$ and the parents of children with CF, only $80 \%$ reported that they had discussed hand hygiene with their CF care team; fewer were told to perform hand hygiene when entering (39\%) and leaving (49\%) the CF clinic. ${ }^{381}$ This study provides just one example of missed opportunities to improve IP\&C education.

The following components of effective education should be incorporated into IP\&C education for CF: (1) developing knowledge, skills, and attitudes; (2) identifying and engaging stakeholders; (3) utilizing positive deviance and early adopters as described below; and (4) performing audits and providing feedback.

Knowledge, skills, and attitudes. The importance of knowledge, skills, and attitudes in educating individuals to change behavior has been described in the CF community ${ }^{381,382}$ as well as for healthcare personnel. ${ }^{383-387}$ Knowledge, or facts, can be taught using didactic or case-based methods at the bedside or in the clinic. Adults learn best if they perceive the relevance of the information to their personal situation and are provided with the rationale. Adult learning is most effective when flexible and when educational methods encourage networking, critical analysis, and reflection on practice and provide an opportunity for open questioning. In contrast, children will benefit from methods that are age appropriate. Provision of 
repeated exposure to educational information is critical to reinforce the principles and allow for questioning. Skills are practical tasks that range from very simple procedures to complex techniques applied to varying circumstances. Observations of adult learners and return demonstrations by adult learners are effective methods to assess skill level and competency to perform tasks independently. Children are also eager to demonstrate their mastery of new skills, and their learning can be validated using a show-and-tell strategy. Attitudes are the products of individual beliefs and professional and personal life experiences. Focus groups and questionnaires are useful tools for defining the beliefs of the target audience and addressing specific attitudes and beliefs; these techniques have been successful with environmental services workers. ${ }^{386}$

Stakeholders. Implementation of the recommendations in this document will likely require many stakeholders to change their practices. Therefore, people with CF, their families, and healthcare personnel must believe that change is necessary. People with CF must believe that change will benefit them. Healthcare personnel must believe that change will benefit their patients and/or themselves in terms of being rewarded for their performance and professionalism. Furthermore, effective education must engage clinical opinion leaders who command the respect of those around them, understand the reasons for change, and can help implement needed change. While senior clinical staff members can serve as role models for needed change, in other settings such individuals may be the most resistant to change. If the latter occurs, focused efforts are needed to engage these individuals and ensure that they understand the importance of the recommended changes in practice and their unique role in modeling these changes. Notably, the lack of positive role modeling among senior clinical staff was cited as a reason for low adherence to hand hygiene practices. ${ }^{388}$

Positive deviance/early adopters. Another successful strategy to effect change is the use of positive deviance or early adopters. Positive deviance is based on the observation that within every community are individuals or groups whose unique behaviors and strategies enable them to find better solutions to problems than their peers, despite having access to the same resources and facing similar or worse challenges. Positive deviance is an asset-based, problem-solving, community-driven approach that facilitates discovery of successful behaviors and strategies and the development of an action plan to promote their adoption by all concerned. ${ }^{389}$ Positive deviance has been used to combat seemingly impossible problems in the community (eg, childhood malnutrition) and in healthcare settings (eg, reduction of MRSA infections and improvement in hand hygiene compliance) ${ }^{390}$ Thus, the CF community can be innovative and can include the positive deviance approach to effect change.

Audits and feedback. Auditing healthcare personnel adherence to recommended practices (eg, hand hygiene and Contact Precautions for patients with MRSA) has become an important part of routine IP\&C and/or quality improvement programs and is required by credentialing organizations, such as The Joint Commission. Feedback may occur at any time and by anyone when a lapse in practice is observed. Trends of audit results should be provided to clinical teams at regular intervals (eg, quarterly, semiannually, or annually). Organizational research has demonstrated that the success of adherence to guidelines is determined by the quality of the feedback. ${ }^{391}$ Feedback should be timely, individualized, nonpunitive, and customizable and should involve the recipients of the feedback in the planning of the feedback program. Thus, collaboration of the CF care team with IP\&C staff to develop an auditing and feedback program is recommended to facilitate implementation of the recommendations in this guideline. The interested reader is referred to a discussion of the theories of feedback interventions. ${ }^{392}$

\section{IV.C. Hand Hygiene}

Hand hygiene is a component of Standard Precautions, which are practices aimed at preventing the transmission of infectious agents. Standard Precautions are based on the principle that all blood, body fluids (eg, sputum and saliva), secretions, excretions (eg, urine, stool, and wound drainage but not sweat), nonintact skin, and mucous membranes may contain transmissible infectious agents. Therefore, containing these potential sources will reduce the risk of transmission of infectious agents. Recommendations for Standard Precautions are based on strong evidence from healthcare settings that has been summarized in the CDC/HICPAC 2007 Guideline for Isolation Precautions. ${ }^{6}$

Hand hygiene is the single most important measure to protect people with $\mathrm{CF}$, healthcare personnel, family members, and friends from transmission and acquisition of potential infectious agents and to prevent contamination of the environment. The CDC/HICPAC and the WHO have published comprehensive guidelines for hand hygiene in healthcare settings, ${ }^{393,394}$ and the updated SHEA/IDSA Compendium of Strategies to Prevent Healthcare-Associated Infections in Acute Care Hospitals, published in 2014, contains information on hand hygiene. Many recommendations in these guidelines can also be applied to nonhealthcare settings, including the home, school, and workplace. Hand hygiene in nonhealthcare settings has reduced respiratory and gastrointestinal tract infections. ${ }^{395,396}$ Hand hygiene opportunities for healthcare personnel, people with CF, and families are summarized in Table 7.

Healthcare personnel should perform thorough hand hygiene, as presented in the figures in the 2009 WHO guideline, ${ }^{394}$ before and after contact with patients and whenever hands are contaminated with respiratory secretions or other body fluids. Contamination may occur from direct patient care activities, from contact with surfaces or equipment in a patient's environment, and/or following coughing or sneezing by healthcare personnel. Use of an alcohol-based hand rub is the preferred hand hygiene method, as these products have 
тавцE 7. Examples of Opportunities for Hand Hygiene by Healthcare Personnel, People with Cystic Fibrosis (CF), and Families

\begin{tabular}{lccc}
\hline & Healthcare personnel & People with CF & Family members \\
\hline Entering CF clinic or hospital room & $\mathrm{X}$ & $\mathrm{X}$ & $\mathrm{X}$ \\
Leaving CF clinic or hospital room & $\mathrm{X}$ & $\mathrm{X}$ & $\mathrm{X}$ \\
Before or after contact with patient & $\mathrm{X}$ & $\mathrm{NA}$ \\
Before and after performing pulmonary function tests & $\mathrm{X}$ & $\mathrm{X}$ & $\mathrm{X}$ \\
After obtaining respiratory tract culture & $\mathrm{X}$ & $\mathrm{X}$ & $\mathrm{X}$ \\
After coughing & $\mathrm{X}$ & $\mathrm{X}$ & $\mathrm{X}$ \\
Before putting on and after removing gloves & $\mathrm{X}$ & $\mathrm{X}$ \\
When hands are contaminated with respiratory secretions & $\mathrm{X}$ & $\mathrm{X}$ \\
Before and after cleaning and disinfecting nebulizer equipment & $\mathrm{X}$ & $\mathrm{NA}$ & $\mathrm{X}$ \\
Before donning gloves for performing sterile procedures & $\mathrm{X}$ & $\mathrm{X}$ \\
After using restroom & & $\mathrm{X}$ \\
\hline
\end{tabular}

Note. X, applicable to this population; NA, not applicable to this population.

${ }^{a}$ If performing activities involving a central venous catheter.

demonstrated greater efficacy in reducing bacterial contamination of hands compared with washing with plain or antimicrobial soap and water. ${ }^{393}$ Alcohol-based hand rubs have excellent activity against gram-positive and gram-negative bacteria, including MDROs, NTM, a variety of fungi, and such viruses as rhinovirus, adenovirus, influenza virus, and RSV. ${ }^{393,394}$ However, in healthcare settings soap and water are used when hands are visibly dirty, sticky, or contaminated with blood or body fluids. If soap and water are used, antimicrobial soap, such as one containing chlorhexidine gluconate, is preferred when caring for people with CF. Use of commercially available antimicrobial soaps in the home is not recommended, as these products do not provide any additional benefits compared with nonantibacterial soaps. ${ }^{397}$

The hands of healthcare personnel caring for people with $\mathrm{CF}$ and the hands of people with CF and their families can become contaminated with CF pathogens due to contact with infectious respiratory secretions. In a study conducted in 7 CF clinics, the hand contamination rate among people with CF ( $n=100$ participants) was $13.5 \%$, and, in addition, $6.3 \%$ of participants without initial detection of CF pathogens contaminated their hands during clinic visits. ${ }^{128}$

Fingernails are of special concern, as the subungual areas of hands harbor high concentrations of bacteria. ${ }^{398}$ Compared with healthcare personnel with natural nails, those wearing artificial nails were more likely to harbor gram-negative organisms on their fingertips before and after hand hygiene. ${ }^{399-401}$ Artificial nails worn by healthcare personnel have been associated with outbreaks of infectious agents, including $P$. aeruginosa. ${ }^{402-405}$ While no specific studies of the role played by artificial nails in the transmission of pathogens in CF have been performed, the clinical experience in ICUs and other healthcare settings can be applied to CF. Thus, healthcare personnel who provide care to people with CF should not wear artificial nails. While studies have not evaluated the risk of artificial nails worn by people with CF or their families, it is prudent to avoid this potential risk factor for acquisition of gram-negative pathogens.

\section{IV.D. Personal Protective Equipment (PPE)}

PPE are wearable barriers intended to protect healthcare personnel from exposure to or contact with infectious agents. PPE includes gloves, gowns, facemasks, respirators, and eye protection (eg, goggles and face shields). Healthcare personnel can wear PPE alone or in combination, based on the anticipated patient interaction and potential for exposure to blood or body fluids or for exposure to known or suspected pathogens. PPE is subject to FDA regulations under the device provisions of the federal Food, Drug, and Cosmetic Act. ${ }^{406}$ $\mathrm{CDC} / \mathrm{HICPAC}$ recommendations for standard and transmission-based precautions provide detailed indications for PPE use. $^{6}$ A summary of the recommended use of PPE by healthcare personnel, people with CF, and their families is provided in Table 8.

\section{IV.D.1. Gowns and Gloves}

Healthcare personnel. As per CDC recommendations, healthcare personnel wear gowns to protect their skin from contact with blood and body fluids and to prevent soiling or contamination of their clothing. Clothing worn by healthcare personnel can be contaminated with MDROs, including MRSA, VRE, and gram-negative bacilli. ${ }^{407-410}$ As per CDC recommendations, healthcare personnel wear gloves for the following reasons: (1) to prevent possible contact with blood or body fluids, mucous membranes, nonintact skin, or other potentially infectious materials; (2) to prevent transmission of pathogens transmitted by the contact route when having direct contact with patients colonized or infected with such pathogens (eg, VRE, MRSA, or RSV); or (3) when handling or touching visibly or potentially contaminated environmental surfaces and patient care equipment. ${ }^{5}$ Gloves worn by healthcare personnel reduce the transmission of viral and bacterial pathogens but do not replace hand hygiene-they are worn in addition to the practice of hand hygiene. ${ }^{411-413}$

For Contact Precautions, the CDC recommends that healthcare personnel don both gown and gloves on room entry for 
тавце 8. Use of Personal Protective Equipment by Healthcare Personnel, People with Cystic Fibrosis (CF), and Families

\begin{tabular}{|c|c|c|c|}
\hline & Healthcare personnel & People with CF & Family members without CF \\
\hline Gowns & $\begin{array}{l}\text { Wear when caring for all people } \\
\text { with CF, per Contact Precautions } \\
\text { and per Standard Precautions }\end{array}$ & $\begin{array}{l}\text { Not recommended } \\
\text { Perform hand hygiene as described } \\
\quad \text { in Table } 7\end{array}$ & $\begin{array}{l}\text { Not recommended routinely } \\
\text { Use as defined in local hospital pol- } \\
\text { icy when visiting hospitalized } \\
\text { patients } \\
\text { Perform hand hygiene as described } \\
\text { in Table } 7\end{array}$ \\
\hline Gloves & $\begin{array}{l}\text { Wear when caring for all people } \\
\text { with CF, per Contact Precautions } \\
\text { and per Standard Precautions }\end{array}$ & $\begin{array}{l}\text { Not recommended } \\
\text { Perform hand hygiene as described } \\
\quad \text { in Table } 7\end{array}$ & $\begin{array}{l}\text { Not recommended routinely } \\
\text { Use as defined in local hospital pol- } \\
\text { icy when visiting hospitalized } \\
\text { patients } \\
\text { Perform hand hygiene as described } \\
\text { in Table } 7\end{array}$ \\
\hline Masks & $\begin{array}{l}\text { Wear surgical (also referred to as } \\
\text { isolation or procedure) mask } \\
\text { when caring for patients under } \\
\text { Droplet Precautions } \\
\text { Wear face shield when splashes are } \\
\text { likely to occur as per Standard } \\
\text { Precautions }\end{array}$ & $\begin{array}{l}\text { Wear surgical (also referred to as } \\
\text { isolation or procedure) mask } \\
\text { when in common areas in health- } \\
\text { care settings (eg, corridors, wait- } \\
\text { ing areas, radiology) } \\
\text { Do not wear when in exam room, } \\
\text { in hospital room, or when per- } \\
\text { forming pulmonary function tests }\end{array}$ & $\begin{array}{l}\text { Not recommended in CF clinics } \\
\text { Use as defined by local hospital pol } \\
\text { icy when visiting hospitalized } \\
\text { patients }\end{array}$ \\
\hline Respirator or PAPR ${ }^{\mathrm{a}}$ & $\begin{array}{l}\text { Wear N-95 respirator or PAPR } \\
\text { when caring for patients under } \\
\text { Airborne Precautions }\end{array}$ & $\begin{array}{l}\text { Not recommended } \\
\text { Wear mask as described above }\end{array}$ & $\begin{array}{l}\text { Wear N-95 to enter room for sus- } \\
\text { pected or confirmed tuberculosis } \\
\text { according to hospital policy }\end{array}$ \\
\hline
\end{tabular}
${ }^{\text {a }}$ Powered air-purifying respirators (PAPRs) are recommended for individuals who are unable to tolerate or pass a fit test for an N-95
respirator.

all contacts with the patient and/or environmental surfaces and patient care equipment that could be potentially contaminated. ${ }^{5}$ For Standard Precautions, an isolation gown is worn only if there is anticipated contact with blood or body fluids. However, application of appropriate Standard Precautions may be inconsistent, because healthcare personnel cannot always anticipate potential contact with infectious body fluids and because PPE are not always readily available. ${ }^{414}$

All healthcare personnel should wear gowns and gloves when caring for all people with CF regardless of respiratory tract culture results. The rationale for the universal use of gowns and gloves by healthcare personnel caring for people with CF is that direct and indirect contact with respiratory secretions that may contain transmissible pathogens is likely to occur, including through contact with contaminated environmental surfaces and equipment. Additional support for this practice is derived from a study in adult medical and surgical ICUs that demonstrated decreased acquisition of MRSA in units where healthcare personnel wore gowns and gloves for all patient contacts and when entering any patient room. ${ }^{407}$

People with CF and families/visitors. There are no data to support a recommendation for people with CF or their families to wear gowns or gloves in healthcare or nonhealthcare settings to prevent the transmission or acquisition of potential pathogens. However, some high-risk units in hospitals may choose to require visitors to wear gowns and gloves.

\section{IV.D.2. Masks, Eye Protection, and Respirators}

Healthcare personnel. As per the CDC recommendations, facemasks are loose-fitting disposable PPE worn by healthcare personnel when caring for patients with known or suspected infections that require Droplet Precautions (eg, influenza, pertussis, or adenovirus infection). Masks, in combination with eye protection, are worn to protect healthcare personnel during procedures and patient care activities likely to generate splashes or sprays of blood, body fluids, or secretions (eg, suctioning, intubation, and operative procedures). These types of masks may be referred to as surgical, procedure, or isolation masks. All facemasks are single use and should be changed whenever damaged, soiled, or damp or if breathing through the mask becomes difficult. While CF pathogens are transmitted patient to patient by the droplet route, such pathogens are not transmitted to healthcare personnel. Thus, healthcare personnel are not required to wear a mask routinely when caring for people with CF unless there is an infection with a known or suspected pathogen that requires Droplet Precautions or according to Standard Precautions as described above. 
A respirator is a tight-fitting device worn on the face, covering at least the nose and mouth, to reduce the wearer's risk of inhaling droplet nuclei containing infectious agents. The Occupational Safety and Health Administration sets regulatory standards for respirator use. ${ }^{415}$ Healthcare personnel who may have exposure to airborne infectious agents are medically evaluated and fit-tested to wear a disposable respirator, usually one designated N95, which means that while breathing through it the filter removes $95 \%$ of airborne particles. Healthcare personnel who cannot wear or be adequately fitted with an N95 respirator may use a PAPR with a disposable hood. Respirators are worn by all healthcare personnel entering the room of a patient under Airborne Infection Isolation for pulmonary $\mathrm{TB}^{4,6}$ Respirators are also worn when performing an aerosol-generating procedure (eg, open suctioning, emergency intubation, or bronchoscopy) on a patient with known or suspected influenza. ${ }^{14}$ Healthcare personnel who will be using an N95 respirator should be fit-tested annually to ensure that the proper size is used.

People with CF and families/visitors. Masks prevent ill individuals from spreading infectious respiratory droplets. For people entering healthcare settings, availability of facemasks is an essential component of respiratory hygiene and cough etiquette, particularly during times of seasonal community-onset respiratory infections (eg, influenza). ${ }^{6}$ Masks are available in adult and child sizes (designed for ages 5-12 years). As described above (Section II), experimental and clinical data have demonstrated the generation of infectious droplets by people with CF; these studies confirm the potential for transmission of CF pathogens to others with CF by the droplet route. ${ }^{1,120-122,125-127}$ Thus, to prevent transmission by the droplet route people with CF should routinely don a facemask of appropriate size when entering healthcare settings where they are likely to encounter others with CF. Such settings include the common areas of the CF clinic, when leaving their hospital room, or when leaving the clinic exam room. However, it is possible that very young children, people in respiratory distress, and people exercising may not be able to tolerate a mask. Such individuals should be instructed to practice other components of respiratory hygiene (ie, cough into a tissue, discard the tissue, perform hand hygiene after coughing, etc) and remain at least 6 feet from others with CF.

Respirators are not recommended routinely for patient use, but the $\mathrm{CDC}$ recommends that visitors wear respirators when entering the room of a patient under Airborne Infection Isolation Precautions with suspected or confirmed pulmonary TB. ${ }^{4}$

\section{IV.E. Environmental Cleaning and Disinfection}

\section{IV.E.1. Overview}

Many studies published in the past decade have demonstrated that contaminated environmental surfaces play a role in the transmission of healthcare-associated pathogens (eg, MRSA, VRE, $C$. difficile, and $P$. aeruginosa) and that improved environmental cleaning and disinfection are effective in reducing the transmission of such pathogens. ${ }^{322-325,416,417}$ The importance of the principles of environmental contamination and cleaning and disinfection can be extrapolated to the $\mathrm{CF}$ clinic where surface contamination has been studied extensively. ${ }^{126-128,317}$ As all people with CF may harbor transmissible pathogens or are at risk of acquiring such pathogens, environmental cleaning and disinfection practices should be implemented when caring for all people with CF.

Four strategies can reduce transmission from contaminated healthcare surfaces and equipment: (1) improve cleaning and disinfection of the rooms of patients known to carry healthcare-associated pathogens after discharge (ie, terminal cleaning); (2) disinfect high-touch surfaces in isolation rooms daily; (3) disinfect portable equipment between patients or use disposable or dedicated equipment in isolation rooms; and (4) expand efforts to improve cleaning and disinfection of all rooms if there is concern that patients harboring MDROs are not identified or are identified after long delays. Automatic disinfection devices (eg, hydrogen peroxide vapor and ultraviolet cleaning) are promising but require additional studies before recommendations for their routine use can be made. ${ }^{416,418}$

Contamination of surfaces most often results from the failure to clean rather than faulty cleaning methods or ineffective products, which supports the importance of education and monitoring adherence. ${ }^{326}$ Standardization of cleaning and disinfecting methods, utilizing educational programs, and checklists and audits can all improve effectiveness. Three types of audits after cleaning and disinfecting have been described: (1) direct observation, (2) use of fluorescein powder or ATP detection methods to demonstrate the removal of potentially infectious pathogens, and (3) bacterial cultures of surfaces. Objective measures utilizing fluorescein dye or ATP detection are the most effective and practical to implement. ${ }^{419,420}$ Since most IP\&C departments are currently engaged in monitoring environmental cleaning, coordination between the CF care team and the IP\&C team is advised. Strategies to enhance the effectiveness of environmental cleaning in both ambulatory and inpatient areas are provided in Table 9. Checklists and other tools for cleaning and additional background information are available on the CDC website. ${ }^{419}$

\section{IV.E.2. Healthcare Facilities: Respiratory Therapy, Nebulizers, and Diagnostic Equipment}

Devices used for respiratory therapy (eg, nebulizers) or for diagnostic evaluation (eg, bronchoscopes and spirometers) are potential reservoirs or vehicles for the transmission of infectious organisms. Routes of transmission may be from a contaminated device to a patient, from a patient to a patient via a contaminated device, or from one body site to the 
TABLE 9. Strategies to Enhance the Effectiveness of Environmental Cleaning in Ambulatory and Inpatient Settings for People with Cystic Fibrosis (CF)

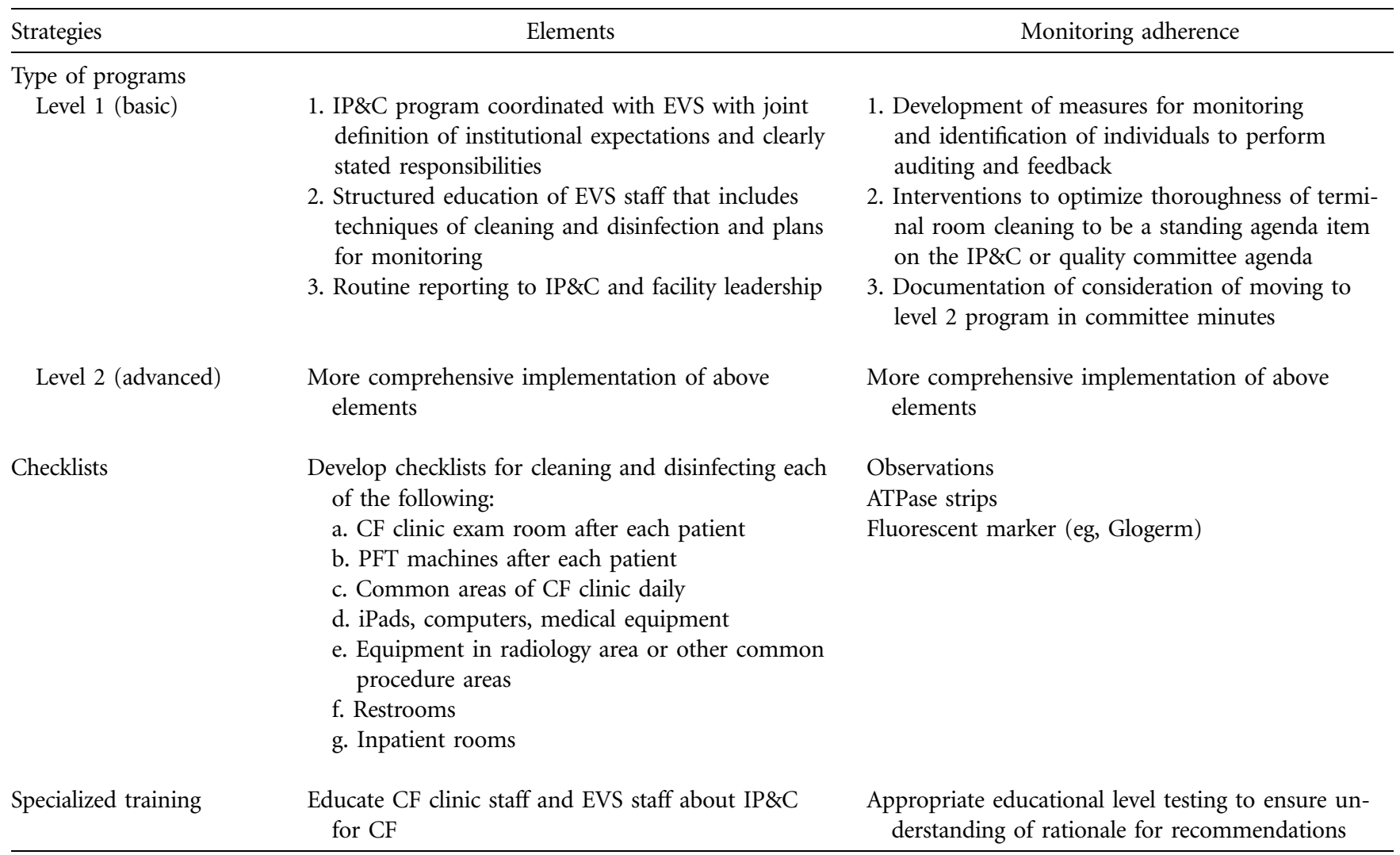

nоте. See the Centers for Disease Control and Prevention website for downloadable tools and informational brochures (http://www .cdc.gov/HAI/toolkits/Evaluating-Environmental-Cleaning.html). EVS, environmental services; IP\&C, infection prevention and control; PFT, pulmonary function test.

respiratory tract of the same patient. Reservoirs of aerosolproducing devices (eg, nebulizers) are subject to overgrowth of bacteria that can be aerosolized during device use. Although a patient's own respiratory flora usually contaminate nebulizers, it is prudent not to introduce those microorganisms into the lower respiratory tract during aerosol treatments. Thus, processes for proper cleaning and sterilization or disinfection of reusable equipment are essential components of a program to prevent infections of people with CF. Evidence-based guidelines for the care of bronchoscopes and other semicritical items have been published, and the latest developments in reprocessing semicritical items were recently reviewed. ${ }^{421}$ Hospitals must follow these recommendations.

Several studies of infections that occurred in association with contaminated respiratory therapy and diagnostic equipment have provided important insights into preventing such infections. These include the following: (1) Strict adherence to aseptic technique is important. (2) Proper training of personnel responsible for reprocessing equipment is important, including demonstration of competency initially and then at least annually, as is consistent adherence to reprocessing guidelines. ${ }^{421}$ (3) Single-dose medication vials are always pre- ferred, due to the risk of contamination ${ }^{349,357,422,423}$ (if multidose medication vials must be used, then the manufacturer's directions for handling, dispensing, and storing must be followed precisely to prevent contamination and the transmission of potential pathogens). (4) Tap water may be used for cleaning nebulizers and other respiratory therapy equipment, but sterile water or water processed by filtration (filter size of less than or equal to 0.2 microns) must be used in the final rinse because tap water and distilled water may be contaminated with CF pathogens. ${ }^{424}$ Sterile water or properly filtered water is recommended for filling respiratory therapy equipment reservoirs (eg, humidifiers), and sterile saline is recommended for sinus rinses. (5) Equipment should be cleaned before disinfection or sterilization to ensure that the sterilization process is maximally effective. ${ }^{7}$ (6) Air-drying equipment after it has been cleaned and disinfected is an essential step prior to storage because items that remain wet provide favorable conditions for bacterial growth. ${ }^{1,7}$

Bacterial contamination of nebulizers used during hospitalizations has been demonstrated. ${ }^{320}$ However, methods of caring for nebulizers in the hospital setting have been widely disparate ${ }^{424-426}$ and have included changing nebulizers every 
2-7 days; changing mouthpieces after each use; and rinsing them with sterile water, drying them, and then placing them in a plastic bag between uses. In the preparation of recommendations for this guideline, discrepancies in published guidelines were noted.

Furthermore, there are limited data to inform recommendations for care of disposable nebulizers used in the hospital. The care of nebulizers was addressed in a study of 30 people with CF admitted for pulmonary exacerbations who received aerosolized bronchodilator therapy 4 times daily. ${ }^{427}$ The nebulizers were not cleaned or disinfected between treatments but were replaced after 24 hours. Cultures of the residual fluid inside the nebulizer cup were obtained before administering successive treatments and prior to discarding the nebulizer after 24 hours of use. None of the 150 nebulizer samples obtained grew CF pathogens. This study did not address the use of disposable nebulizers for longer than 24 hours.

Important principles for the care of disposable nebulizers used in the hospital for individuals with CF are as follows: (1) nebulizers are for use in a single patient only; (2) when handling the nebulizer and dispensing the medications, aseptic technique should be followed; (3) nebulizers should be handled away from sinks to prevent contamination; (4) only sterile water should be used for rinsing nebulizers; (5) after each use, residual volume should be rinsed out with sterile water, and masks/mouthpieces should be wiped with an alcohol pad; and (6) nebulizer contamination between uses can be avoided by not placing nebulizers in line with the ventilator circuit, thereby exposing the nebulizer to tubing condensation. The safety of storing moist nebulizers in plastic bags is unknown. Durable, nondisposable nebulizers used in the hospital can be processed in a central sterilization area according to the methods described below for home use (Section IV.E.4) if they can be returned to the same patient in time for their next treatment.

\section{IV.E.3. Nonhealthcare Settings: Nebulizers}

Although no published reports have definitively proven that CF pathogens were acquired from contaminated equipment during home therapy, bacterial contamination of home nebulizers of people with $\mathrm{CF}$ has been documented in several studies. ${ }^{428-432}$ Additionally, cleaning and drying home respiratory therapy equipment between uses was associated with a decreased risk of acquiring B. cepacia complex. ${ }^{1}$ In a study of experimental contamination of nebulizers, hot water and soap effectively removed most inoculated bacteria. ${ }^{431}$ However, these experimental conditions may not mimic true use by people with $\mathrm{CF}$, and, as described above, potential pathogens from environmental sources (eg, tap water) may contaminate equipment inadvertently and thereby cause infection.

Respiratory care equipment used in the home (eg, nondisposable nebulizers) is durable and designed for long-term use. Thus, to prevent infections caused by contaminated respiratory therapy equipment used in the home, equipment should be cleaned, disinfected, and air-dried after each treatment. (1) Equipment must be cleaned well to remove all organic and inorganic debris before disinfection. After cleaning with dish soap and water, disinfect either by immersion in cold disinfectants or by heat, if permissible by the manufacturer. ${ }^{7}$ Dried or baked debris on equipment makes removal more difficult, and the disinfection process becomes less effective or even ineffective. ${ }^{433,434}$ (2) Equipment must be disinfected by either heat or cold disinfectant methods, as permissible by the manufacturer. Heat methods include immersion in continuously boiling water for 5 minutes; washing in a dishwasher if the equipment is dishwasher safe and the water achieves a temperature greater than $158^{\circ} \mathrm{F}\left(70^{\circ} \mathrm{C}\right)$ for 30 minutes; $^{7}$ use of a microwave oven if the equipment is microwave safe and can be placed in a bowl of water in a home microwave oven $(2.45 \mathrm{Ghz})$ for 5 minutes; ${ }^{435-437}$ or use of electric steam sterilizer (eg, baby bottle sterilizer) ${ }^{438}$ Cold methods include soaking in 70\%-90\% ethyl or isopropyl alcohol for 5 minutes (avoid use near open flames) or in 3\% hydrogen peroxide for 30 minutes. ${ }^{439,440}$ These preparations will lose activity over time, and the optimal storage time is unknown. Vinegar (acetic acid) is not recommended because it has inadequate activity against some potential CF pathogens (eg, S. aureus). ${ }^{7,441,442}$ Bleach is no longer recommended because a $0.5 \%$ hypochlorite solution did not reduce the number of CF pathogens on home nebulizers. ${ }^{443}$ Benzyl ammonium chloride (Control III) is also not recommended for use because it has a narrow spectrum of activity and is slow in action. Additionally, outbreaks have been related to contamination of this agent. ${ }^{344}$ (3) Equipment should be rinsed after use of the cold disinfectant with either sterile water or filtered (less than or equal to 0.2 microns) water, as described above. Sterile water can be prepared by boiling tap water and achieving a rolling boil for 5 minutes. Sterile water can become contaminated after use and/or storage, but the frequency of this is unknown. Boiling water immediately before use minimizes this possibility. Distilled water is not recommended for cleaning or rinsing respiratory therapy equipment since contamination with B. cepacia complex can occur during the manufacturing process. ${ }^{355}$

\section{IV.F. Strategies for CF Clinics}

Several epidemiologic studies have provided evidence for potential transmission of CF pathogens in CF clinics, as described above (Section III.A). While the risk of transmission in CF clinics cannot be quantified, the health benefits of $\mathrm{CF}$ clinics clearly outweigh the risks of acquisition of CF pathogens. The IP\&C recommendations for CF clinics are detailed below and in Table 10.

\section{IV.F.1. Cohort Segregation versus All-Patient Separation}

Many CF centers throughout the world practice cohort segregation, whereby separate clinic sessions are held for people with CF who are infected with the same pathogen. For ex- 
ample, there is a separate clinic for people infected with MRSA or a separate clinic for people infected with an epidemic strain of $P$. aeruginosa. ${ }^{1,161,185,374,375,444-446}$ While cohort segregation has been associated with a reduced incidence of LES of $P$. aeruginos $a^{185,373,375,444}$ and a decrease in the incidence and prevalence of Burkholderia spp. in the United States, ${ }^{44}$ additional IP\&C practices were also implemented at the same time. Concurrent practices included recommending no socialization among people with CF in nonhealthcare settings, alternatives to common waiting rooms, removal of common toys and books, an emphasis on hand hygiene, single-use mouthpieces for PFT equipment, and aggressive eradication protocols for P. aeruginosa. ${ }^{375,44-446}$ Therefore, it is difficult to conclude which IP\&C practices were most important for successfully decreasing transmission.

Furthermore, maintaining cohort segregation is difficult, as people with CF may (1) harbor more than 1 pathogen, (2) need urgent care, or (3) have newly identified pathogens that could change their cohort status. Additionally, respiratory tract cultures do not always accurately detect CF pathogens, and thus all people with CF could harbor potentially transmissible pathogens. Specimens obtained from the upper airway lack sensitivity and specificity for the lower airway. ${ }^{42}$ Despite standardized protocols for processing CF specimens, potential pathogens may escape detection due to low organism burden, overgrowth by other species, or misidentification. ${ }^{1,447}$ Genotyping studies have also demonstrated the potential for replacement of an initial infecting Burkholderia strain with another strain (ie, superinfection) when CF patients have been segregated into groups on the basis of infection status. ${ }^{159,448}$

Many clinicians in centers implementing cohort segregation have noted the cost, stigmatization, and psychosocial stresses associated with this practice and emphasize that cohort segregation should be considered only when there is strong epidemiologic and genotypic evidence for transmission in the CF clinic. ${ }^{185}$ Thus, cohort segregation could be implemented on a case-by-case basis if ongoing transmission of a CF pathogen occurs despite implementation of the IP\&C recommendations detailed in this guideline.

To date, studies have not compared the impact of cohort segregation versus separation of all patients with CF from each other. Given the lack of definitive support for cohort segregation and the complexity and shortcomings of implementing cohort segregation, recommendations include separation of all people with $\mathrm{CF}$, regardless of their respiratory tract culture results, and practicing the IP\&C recommendations detailed in this guideline. Additionally, given the adverse clinical impact of many CF pathogens, including MRSA, epidemic strains of P. aeruginosa, and Burkholderia spp. (Section III.A), and the insensitivity of respiratory tract cultures to accurately detect all CF pathogens, ${ }^{1}$ maintaining a separate cohort for Burkholderiainfected individuals is not recommended.

\section{IV.F.2. CF Clinic Logistics}

As previously described, infectious droplets can travel 6 feet (2 meters; Section II.C). ${ }^{6}$ Thus, all people with CF (unless they live in the same household) should be separated by at least 6 feet (2 meters) from others with CF to reduce the risk of droplet transmission. ${ }^{128,447}$ Strategies to schedule and manage people with CF in clinics must include minimizing waiting time in a common reception area or waiting room. Such strategies could include placement of the individual with CF in an exam room on arrival, use of a pager system if a room is unavailable, a staggered clinic schedule, portable pulmonary function testing, and rotating CF team members into the exam room (Table 9).

\section{IV.F.3. Hand Hygiene}

People with $\mathrm{CF}$ and their accompanying family members should perform hand hygiene on entering and leaving a CF clinic as well as throughout the clinic visit, as contact with respiratory secretions can occur during coughing, sneezing, or contact with contaminated environmental surfaces or equipment. Hand hygiene recommendations for healthcare personnel, people with CF, and their families are described in Section IV.C and in Table 7. To promote hand hygiene, all ambulatory areas should have appropriate supplies for hand hygiene, including conveniently placed alcohol-based hand rub in the entryway into the $\mathrm{CF}$ clinic, the waiting room, exam rooms, PFT laboratories, and restrooms. ${ }^{6,393,394}$ Furthermore, when hand hygiene is not witnessed, people with $\mathrm{CF}$ and their families should be empowered to remind healthcare personnel to perform hand hygiene.

\section{IV.F.4. Mask Use by People with CF}

In the 2003 Infection Control Guideline for CF, the routine use of masks by people with CF was an unresolved issue because of a lack of supporting evidence that masks prevented transmission of CF pathogens. In a survey of IP\&C practices conducted in CF centers in the United States in 2005, some centers $(27 / 76[35 \%])$ used masks in the ambulatory setting, but the benefits of this practice had not been studied. ${ }^{449}$ However, recent studies have found infectious droplets in the air of CF clinics (Section III). This supports the use of masks by people with CF to both contain infectious droplets and prevent acquisition of potential pathogens. ${ }^{127,128}$

Thus, all people with CF should wear a mask of appropriate size in healthcare facilities to reduce droplet transmission and acquisition of CF pathogens. Mask use by people with CF is consistent with CDC recommendations to prevent droplet transmission of pathogens by infected patients and to prevent the acquisition of potential pathogens by susceptible individuals. Masks should be worn throughout the healthcare facility unless the individual with $\mathrm{CF}$ is in an exam room or performing PFTs. ${ }^{6}$ To facilitate mask use, all CF clinics should provide masks of different sizes on entry into the clinic. Some centers have chosen to implement mask use via respiratory 


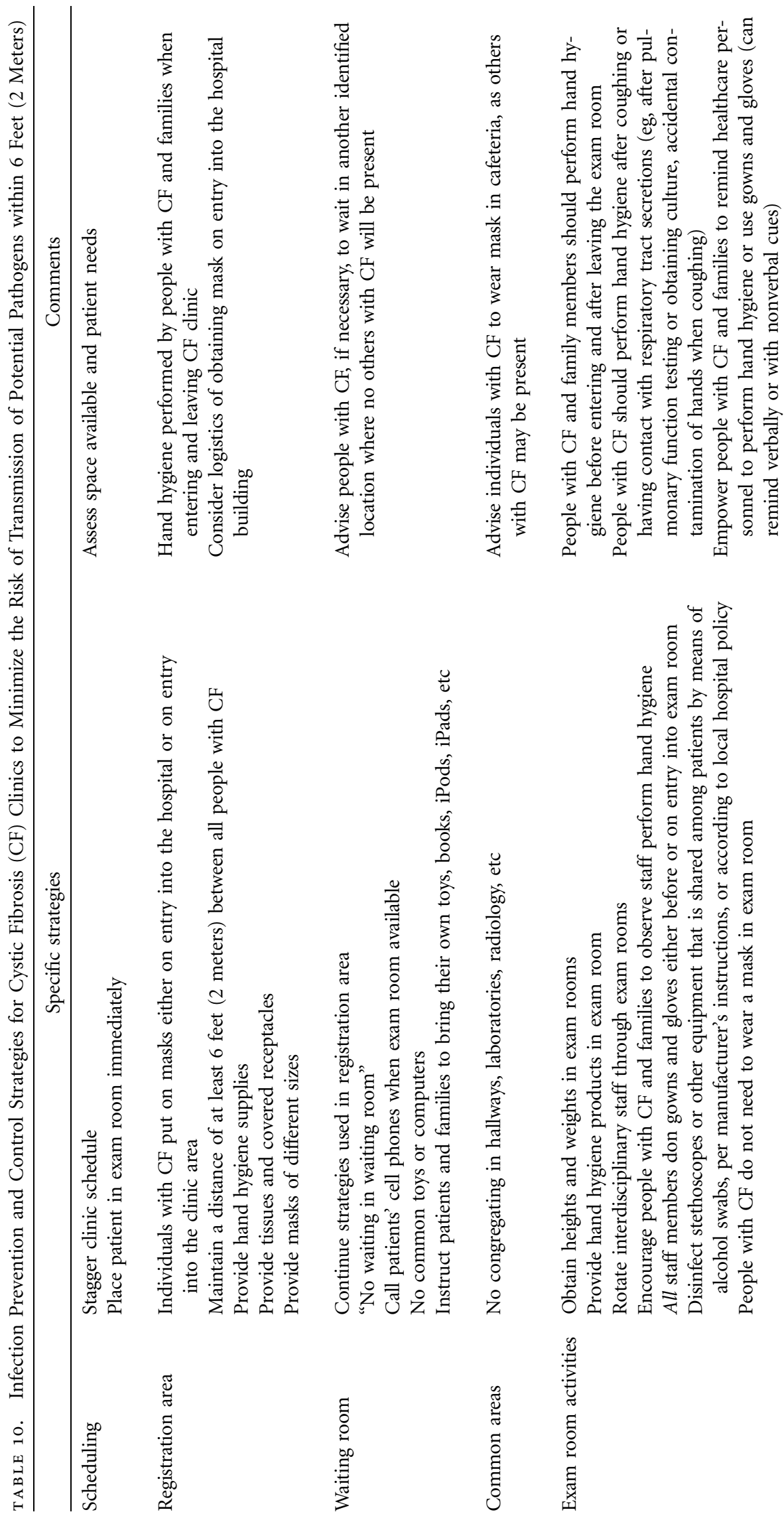



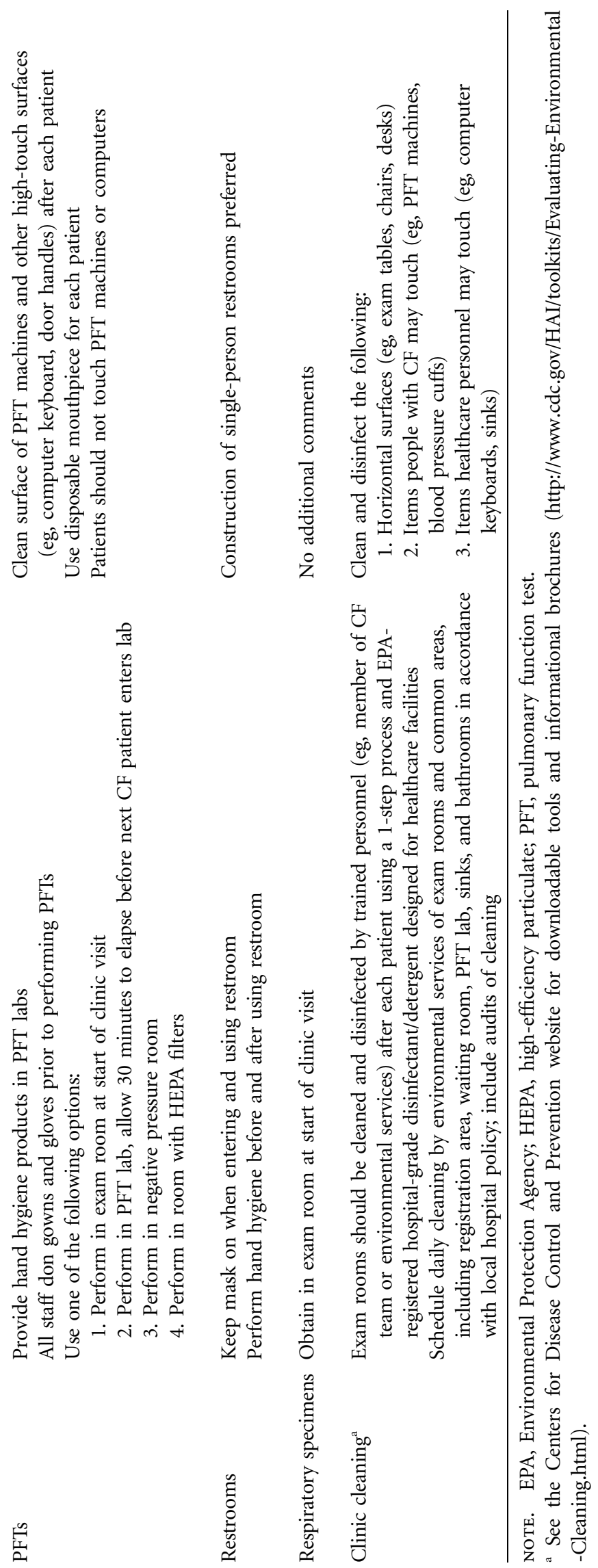
hygiene stations on entry into the facility. Reminders, including signs in ambulatory settings, can instruct individuals with CF to contain their secretions, that is, to wear a mask, cough into a tissue, immediately discard the soiled tissue into a trash receptacle, and perform hand hygiene after contact with respiratory secretions. ${ }^{6,450}$

\section{IV.F.5. Gown and Glove Use by Healthcare Personnel}

Gowns and gloves should be worn by all healthcare personnel caring for all people with CF in healthcare settings. The use of gowns and gloves by healthcare personnel in CF clinics is consistent with the principles of Contact Precautions and Standard Precautions (Section IV.D.1), as all individuals with CF may harbor potentially transmissible pathogens. By wearing gowns and gloves when caring for people with CF, healthcare personnel protect their clothing and hands from possible contamination due to direct or indirect contact with respiratory secretions and thus avoid serving as vehicles for the transmission of CF pathogens. To facilitate the use of gowns and gloves, all CF clinics must ensure ready availability of gowns and gloves of different sizes in a location convenient to room entry so they can be put on prior to entering the room. They should be removed in the room and discarded in a covered receptacle. Furthermore, people with CF and their families should be empowered to remind healthcare personnel to wear appropriate PPE on entry into their rooms.

\section{IV.F.6. Pulmonary Function Testing}

PFTs often generate coughing and involve the use of common equipment. Thus, IP\&C practices when performing PFTs must be implemented to minimize the transmission of CF pathogens by contact with contaminated equipment and/or by infectious droplets. Hand hygiene should be performed by healthcare personnel and people with CF before and after performing PFTs to prevent hand contamination by potential pathogens. Healthcare personnel performing PFTs should wear gowns and gloves (changing these after each patient) to prevent soiling of clothing and hand contamination with potential pathogens. Droplets can be detected at least 6 feet from people with CF performing PFTs, and these droplets clear the air 30 minutes after performing PFTs. ${ }^{125}$ To minimize exposure to infectious droplets, PFTs should be performed using one of the following options: (1) in the exam room at the beginning of the clinic visit, allowing 30 minutes to elapse between CF patients; (2) in a negative pressure room (AIIR); (3) in a PFT laboratory with HEPA filters; or (4) in a PFT laboratory without HEPA filters, allowing 30 minutes to elapse between individuals with CF.

HEPA filters remove $99.97 \%$ of particles (more than or equal to 0.3 microns in diameter) from the air that passes through the filter. HEPA filters are used to protect hematopoietic stem cell transplant recipients in a Protective Environment from mold infections and can be used to prevent the spread of airborne bacterial and viral infections if air is recirculated in AIIRs. ${ }^{6}$ HEPA filters can be located centrally in the air handler that supplies a specific unit or building or may be located at the point of use within a room. HEPA filters must have a preventive maintenance program of monitoring and replacement in accordance with the manufacturer's recommendations to ensure continued filtration efficiency ${ }^{451}$ HEPA filters with metal frames are recommended rather than those with wood frames, which can get wet and become contaminated with potential pathogens. The effectiveness of a portable HEPA unit depends on (1) room configuration, (2) the amount of furniture and people in the room, (3) placement of the unit, and (4) location of the air supply and exhaust registers. ${ }^{2}$ Portable HEPA units should be capable of recirculating all or nearly all of the room air through the HEPA filter and should achieve the equivalent of more than or equal to 12 air exchanges per hour. ${ }^{452}$ The facility's engineering department can assist with information regarding air changes.

Ultraviolet germicidal irradiation (UVGI) has been used as an adjunct air-cleaning measure in healthcare settings to reduce transmission of bacterial and viral infections. ${ }^{2}$ UVGI can be used within air handling units to disinfect air prior to recirculation or as upper room air irradiation (ie, lamps suspended from the ceiling or mounted on the wall). Implementation of upper room air irradiation requires air mixing between the lower patient care area and the upper room air. There is also concern about the potential for UV light to damage the eyes of people in the rooms. Regular maintenance of UVGI systems is required and includes keeping the bulbs free of dust and replacing old bulbs when needed. Many experts do not recommend UVGI as a substitute for HEPA filtration. ${ }^{2}$

\section{IV.F.7. Environmental Practices}

In CF clinics, there are numerous opportunities for contamination of environmental surfaces and equipment, such as exam tables, PFT equipment, and high-touch objects (eg, doorknobs). Thus, healthcare personnel in CF clinics and PFT laboratories must ensure that the equipment and horizontal surfaces that people with CF may touch are cleaned and disinfected after each CF patient by using a 1-step process and EPA-registered hospital-grade disinfectant/detergent designed for housekeeping. ${ }^{1}$ This includes cleaning and disinfecting common equipment used for individuals with CF (eg, stethoscopes, demonstration equipment for chest physiotherapy, pulse oximeters, and the outside of PFT equipment). The same principles of cleaning, disinfecting, and auditing of cleaning presented in Section IV.E.2 and the tools presented in Table 10 apply to CF clinics and PFT laboratories. Environmental service personnel trained in the principles of $\mathrm{CF}$ IP\&C should be available during the hours that people with $\mathrm{CF}$ are cared for in the ambulatory clinic to assist the CF care team to ensure appropriate environmental cleaning and disinfection. 
Use of common items, such as toys, books, pens, and computers, should be avoided in CF clinics. Instead, patients and families should be encouraged to bring such items with them to the clinic for their own personal use. A recent study addressed the potential of mobile handheld devices (MHDs; eg, iPads) to serve as reservoirs for potential pathogens and created the iPBundle. ${ }^{453}$ The iPBundle includes (1) use of a waterproof, nonporous MHD case; (2) disinfection of the MHD as per institutional policies for noncritical items; (3) regular disinfection by setting an alarm on the MHD; and (4) hand hygiene before and after MHD use.

\section{IV.F.8. Use of Restrooms}

In the 2003 Infection Control Guideline for CF, use of common restrooms in ambulatory settings was an unresolved issue. ${ }^{1}$ While no studies have shown acquisition of potential CF pathogens from restrooms, these are common areas in ambulatory settings, including CF clinics. Thus, people with CF should wear a mask while using the restroom and should perform hand hygiene before and after using the restroom. Education, providing proper EPA-registered hospital-grade disinfectant/detergent, and signage describing these practices can facilitate adherence to this recommendation. When new clinic areas are designed, single-person-use restrooms are preferred.

\section{IV.G. Hospital Room Placement and Transmission- Based Precautions}

Single-patient rooms with bathrooms that are not shared with other patients are preferred for all non-CF and CF patients who may harbor MDROs to reduce the risk of transmission. ${ }^{5,6}$ For people with CF, it is reasonable for individuals who reside in the same household to share a room. The following types of isolation are relevant when caring for people with CF.

Contact Precautions are intended to prevent direct and indirect transmission of infectious agents between patients, prevent transmission from patients to healthcare personnel, and prevent transmission from healthcare personnel to patients. On the basis of the knowledge that we now have about person-to-person transmission of CF pathogens (Section III.A) and the possibility of a person with CF harboring an undetected pathogen, Contact Precautions are recommended for ALL hospitalized people with CF. When caring for patients under Contact Precautions, all healthcare personnel must put on a gown and gloves on entry into the room and discard the gown and gloves before exiting the room. Availability of PPE either outside the room or within the room at the point of entry is necessary to ensure adherence to the recommended use. Since bacterial contamination of surfaces and equipment in a patient room can occur, gown and gloves are recommended even if healthcare personnel do not intend to touch the patient. Medical equipment (eg, stethoscopes or blood pressure cuffs) should be dedicated to the patient while under Contact Precautions. Cleaning and disinfection of surfaces and equipment should be performed according to hospital policy. A surgical (procedure or isolation) mask or face shield should be added according to Standard Precautions if there is likely to be a splash of respiratory secretions.

Droplet Precautions are intended to protect healthcare personnel from becoming infected by pathogens transmitted by the droplet route (eg, influenza virus, adenovirus, Bordetella pertussis, or M. pneumoniae). When caring for patients under Droplet Precautions, all healthcare personnel must wear a surgical (procedure or isolation) mask on entry into the patient room. Droplet Precautions are recommended only when caring for people with CF with suspected or proven infection with pathogens that could cause disease in healthcare personnel and are spread by the droplet route. People with CF who require Droplet Precautions will also be under Contact Precautions; therefore, gowns and gloves in addition to a mask will be required on entry into the room.

Airborne Infection Isolation is intended to protect healthcare personnel, other patients, and visitors from contracting infections transmitted by droplet nuclei (eg, M. tuberculosis, varicella-zoster virus, or measles virus). All healthcare personnel entering an AIIR that is housing patients with proven or suspected TB must wear an N95 respirator or a PAPR (Section IV.D.2). AIIRs utilize engineering controls to prevent airborne transmission of infectious agents that remain suspended in the air and travel long distances along air currents. ${ }^{2}$ AIIRs that have been renovated or constructed since 2001 must have more than or equal to 12 air exchanges per hour, and those renovated or constructed prior to 2001 must have more than or equal to 6 air exchanges per hour. AIIRs must be under negative pressure (eg, the direction of the airflow from the corridor is into the room). Preferably, the air in an AIIR is exhausted to the outside, but it can be recirculated if filtered through a HEPA filter.

People with CF who are positive for AFB for the first time are more likely to have NTM than TB. However, while TB is uncommon in people with $\mathrm{CF}$, it can occur in those who live in geographic locations with $\mathrm{TB}$ or with a history of potential risk factors for $\mathrm{TB}$, including exposure to others with $\mathrm{TB}$, foreign birth, or foreign travel to countries with high rates of TB. Airborne Infection Isolation is recommended for people with CF until NTM has been confirmed and $M$. tuberculosis has been ruled out. However, in consultation with local IP\&C staff, the use of AIIR can be guided by a risk assessment if the person who is AFB positive has no risk factors for TB and lives in a geographic location with a very low incidence of TB. Furthermore, if there is evidence within a CF center that NTM are being transmitted by the airborne route, then people infected with NTM should be placed in AIIRs whenever they are hospitalized. People with CF who require Airborne Infection Isolation will also be under Contact Precautions; therefore, gowns and gloves in addition to an N95 respirator will be required on entry into the room. 


\section{IV.H. Construction and Renovation}

CDC recommendations for preventing infection by filamentous fungi during construction, renovation, remediation, repair, and demolition should be followed in all healthcare facilities. ${ }^{2} \mathrm{CDC}$ recommendations for preventing nosocomial aspergillosis and managing potential outbreaks of aspergillosis should be followed by institutional IP\&C departments. ${ }^{372,424}$

\section{IV.I. Nonhealthcare Settings}

Applying the IP\&C principles developed for healthcare settings in nonhealthcare settings is challenging. The information provided in this section is intended to assist people with $\mathrm{CF}$ and their family and friends to make prudent decisions for their activities outside healthcare settings. An IP\&C guideline for individuals residing in Ronald McDonald Houses or similar residential facilities is one example of how IP\&C in healthcare settings may be adapted to other settings. ${ }^{454} \mathrm{Fam}$ ilies of children with CF can now expect such facilities to be knowledgeable about the importance of separating people with $\mathrm{CF}$ from each other and can expect this accommodation. Families should be empowered to request this accommodation if it is not in place.

\section{IV.I.1. Camps and Educational Retreats}

In historic descriptions of CF camps or educational overnight retreats involving more than 1 person with $\mathrm{CF}$, many opportunities for transmission of CF pathogens existed. Close contact between individuals with CF was difficult to avoid, and activities causing coughing were common. Given the strong evidence of person-to-person spread of CF pathogens in CF camps and educational retreats, people with CF should not participate in these settings with others with CF. ${ }^{151,45-457}$ However, people with $\mathrm{CF}$ are encouraged to attend camps and retreats with individuals without CF.

\section{IV.I.2. Indoor Events}

Given the risks of person-to-person spread of CF pathogens within healthcare and nonhealthcare settings, the risk of transmission of CF pathogens between people with CF who attend indoor events at the same time is also present. While the risk of transmission of CF pathogens appears to be greater with epidemic strains of specific pathogens (Section III), the risk cannot be quantified for specific microorganisms. Additionally, the risk of transmission is likely to be higher in small enclosed spaces (eg, in a car or small conference room), but the risk associated with specific indoor events cannot be quantified. Furthermore, there are opportunities for individuals with CF to have inadvertent contact within indoor event spaces (eg, in elevators, at vendor booths, in hallways, or in restrooms). Thus, it is recommended that only 1 person with $\mathrm{CF}$ attend indoor events and that accommodations for nonface-to-face contact, such as webcasts or teleconferences, be encouraged.

\section{IV.I.3. Outdoor Events}

Many of the concerns related to the transmission of CF pathogens due to contamination of surfaces or droplet transmission are minimized at outdoor events. However, if more than 1 person with CF attends such an event, they should be separated by at least 6 feet, should avoid congregating in common areas, should avoid participating in common activities (eg, face painting or meals), and should not travel to the event in the same vehicle unless they reside in the same household. Some CF centers offer strategies to avoid inadvertent contact, for example, identifying people with CF to one another by wearing a colored shirt or a large decorative pin or by providing box meals rather than open buffets.

\section{IV.I.4. Schools}

The risk of transmission of CF pathogens associated with school if more than 1 child with CF attends the same school is unknown. People with CF and their parents or legal guardians are not obligated to disclose the diagnosis of CF or the results of respiratory tract cultures to school personnel. Such information must be maintained as confidential medical information unless the person with CF and/or his or her parent or legal guardian choose to make this information known. However, the student with CF whose diagnosis is disclosed will benefit from the disclosure, as school personnel can then be educated about CF and IP\&C principles. This educational process can ensure the implementation of IP\&C practices that will benefit all students and staff (eg, hand hygiene and respiratory hygiene practices). Thus, provisions for hand hygiene and respiratory hygiene must be available for all students and for school personnel.

If more than 1 child with CF attends the same school, disclosure of the diagnosis of CF will allow schools to make recommended accommodations to minimize the risk of transmission of CF pathogens. Students with CF and their families should discuss these recommendations with school leadership to ensure that accommodations can be implemented within a specific school and then work closely with school personnel to determine how best to implement them. The following accommodations can minimize the risk of transmission of CF pathogens: (1) Students with CF should be placed in separate classrooms. If they must use the same classroom at different times, they should not use the same desk or work station. (2) Students with CF should be assigned different restrooms, encouraged to carry their own water bottles, avoid using public water fountains or use different water fountains, and have lockers as far as possible from each other. (3) Students with CF should be scheduled separately for common activities, including lunch, physical education, and recess. (4) Students with CF should be assigned to separate offices to report for routine medications or if they become ill while at school. (5) Students with CF should not be excluded from group activities, such as large assemblies or pep rallies. Such activities are a crucial part of school life, academic 
development, and socialization. Students with CF should enter and leave the communal areas using different routes from one another and sit as far apart as possible.

Because each school setting is different, strategies may differ from school to school. Many of the strategies recommended for schools may be applicable to day care centers or university settings as well. Additional strategies for schools can be found at the CF Foundation's website (http://www .cff.org).

\section{IV.I.5. Prevention of Viral Transmission and Immunizations}

Strategies to prevent all people (including those with CF) from acquiring respiratory pathogens include (1) hand hygiene, (2) respiratory hygiene, (3) routine vaccinations, (4) influenza vaccinations, and, when relevant, (5) antiviral chemoprophylaxis for influenza (eg, oseltamivir). ${ }^{458}$ Antiviral treatment for influenza should be initiated as early as possible to shorten the duration of symptoms and reduce influenza complications. Antiviral agents are approximately 70\%-90\% effective in preventing influenza and serve as useful adjuvants to vaccination. People without $\mathrm{CF}$ who have acute respiratory illness should avoid close contact with individuals with CF and not share personal items.

Vaccinations for people with CF. Vaccines are critically important for people with CF to maintain their health and minimize the risk of morbidity and mortality from vaccinepreventable illness. The ACIP has guidance for age-appropriate vaccination schedules that is updated each year. ${ }^{115,459,460}$ Additional updates are published as needed throughout the year. People with CF should receive the same recommended vaccinations as people without $\mathrm{CF}$; many recommended vaccines prevent respiratory tract infections that could exacerbate CF lung disease, including influenza, pertussis, and pneumococcal vaccines. ${ }^{271}$ The CF care team should be informed about changes in immunization recommendations. The most recent update affecting people with $\mathrm{CF}$ is the expanded use of pneumococcal conjugate vaccine 13 in individuals more than 2 years of age with chronic lung conditions. ${ }^{461}$

Currently, there are no commercially available vaccines for such CF bacterial pathogens as $P$. aeruginosa or $S$. aureus. Trials have not found investigational vaccines to be efficacious. $^{462-464}$

Vaccinations for family members and close contacts of people with CF. Vaccination of family members and close contacts of people with CF can help to protect people with $\mathrm{CF}$ from vaccine-preventable illnesses. Annual influenza vaccine is recommended for all family members and close contacts of people with $\mathrm{CF}^{465}$ This is especially important among the close contacts of infants with CF who may be too young to receive some vaccines, particularly influenza vaccine. The term "cocooning" describes this concept and is advocated for pertussis and influenza. ${ }^{466}$ For example, women immunized against influenza during pregnancy had fewer respiratory tract illnesses in the 6 months after delivery, and their infants had fewer febrile illnesses. ${ }^{467,468}$ There are also guidelines for vaccination of people who are immunocompromised that are applicable to individuals with CF who have undergone transplantation and their close contacts. ${ }^{469}$

Vaccinations for healthcare personnel. Recent ACIP guidelines have expanded recommendations for healthcare personnel. ${ }^{459,460}$ These recommendations are relevant for the $\mathrm{CF}$ care team and include immunization or immunity to mumps, measles, rubella, varicella, pertussis, and hepatitis B as well as annual influenza immunization. ${ }^{465}$

\section{Healthcare Personnel with CF}

More and more people with CF are living longer, productive adult lives. The following findings reported in the 2012 CFFPR demonstrate the growing need for career counseling for adolescents and young adults: (1) the median predicted survival in 2012 was 41.1 years, nearly 10 years longer than the 31.3 year median survival reported in 2002 ; (2) $49 \%$ of individuals in the CFFPR were 18 years of age or older; and (3) nearly $45 \%$ of adults with CF were employed $(11.8 \%$ were employed part time, and $33.6 \%$ were employed full time). ${ }^{21}$

Six studies have reported that $50 \%$ of the CF population studied was working and that approximately $50 \%$ of those individuals were working in professional occupations, although there was no specific information on healthcare professions. ${ }^{470}$ Similarly, a 2003 monograph containing general information on CF in the workplace ${ }^{471}$ did not specifically address healthcare professions. Guidelines for adult CF care programs in the United States, ${ }^{472}$ for nurses participating in this care transition in Ireland, ${ }^{473}$ and a recent study of the health outcomes of adults who transitioned from pediatric to adult care centers ${ }^{474}$ did not discuss providing career counseling to individuals with CF who were interested in healthcare professions.

While anecdotal experiences suggest that healthcare professions have attracted individuals with $\mathrm{CF}$, few published data exist to inform decisions on choosing a career in healthcare. In the United Kingdom, a survey of adults with CF conducted in 1994 found that $6.6 \%$ of respondents worked in healthcare or closely related professions. ${ }^{475}$ Ten CF associations and prominent $\mathrm{CF}$ center directors worldwide were surveyed, and none of the 4 countries responding had a written policy for managing healthcare personnel with $\mathrm{CF}^{476} \mathrm{~A}$ French association reported that $19 \%$ of adults with CF were working in service fields (eg, education, healthcare, and social care). ${ }^{476}$ The relevance of these studies is uncertain; all were conducted prior to the recent era of improved outcomes for adults with CF.

There are no published reports of transmission of infectious agents between healthcare personnel with CF and their patients. Thus, the frequency of such events is likely to be low and goes unrecognized or unreported. Thus, current rec- 
ommendations for healthcare personnel with CF must be based on common sense, prudence, and individual health status. An editorial and a review article recommended the following considerations for an individual with CF selecting a healthcare profession: (1) the infectious risk to healthcare personnel with $C F$ from healthcare exposures will vary according to the patient population cared for and the healthcare environment; (2) the infectious risks to patients from healthcare personnel with CF will vary according to the severity of CF disease, frequency of coughing, types of CF pathogens, and the ability of the individual with CF to follow sourcecontainment recommendations; and (3) individuals with CF should consider the physical challenges and the ability to perform CF treatments during and after training when selecting a healthcare profession. ${ }^{46,477}$

The only definite restriction for healthcare personnel with $\mathrm{CF}$ is that he or she should not work with patients with $\mathrm{CF}$ or other healthcare personnel with CF. With the stricter IP\&C practices recommended in this guideline, it will become more challenging for people with CF to work in a healthcare environment where other people with CF receive care. For example, it is not feasible that healthcare personnel with $\mathrm{CF}$ could wear a mask routinely in healthcare settings. In contrast, such individuals should wear masks as previously described for all healthcare personnel (Section IV.D.2) and when they are in areas frequented by people with CF. Family members of individuals with CF can work in healthcare professions without restrictions.

Adolescents and young adults interested in healthcare professions should seek advice from members of their CF care team to learn about lower-risk options and to receive advice based on their own health status. This type of counseling should be incorporated, when relevant, into programs for transition from pediatric to adult CF care.

Individuals with CF who work in a healthcare profession are encouraged to disclose their diagnosis to their occupational health service to determine the safest work assignment. The occupational health service must comply with Health Insurance Portability and Accountability Act requirements ${ }^{478}$ as relevant. The employment laws that govern the protections and procedures for healthcare personnel with $\mathrm{CF}$ include the Americans with Disabilities Act and section 504 of the Rehabilitation Act as summarized in the 2003 Infection Control Guideline for CF. ${ }^{1}$

\section{Psychosocial and Medical Impact of Transmission-Based Isolation Precautions}

Acknowledging the potential psychosocial and medical impact of IP\&C recommendations for people with CF, their families, and the CF care team is critical to overcome challenges to implementation and to promote consistent implementation of the recommendations. While few studies have been performed in CF, there are several relevant studies performed in other patient populations.
Studies among non-CF patients. Studies have examined the impact of transmission precautions among non-CF patients with MRSA, TB, or SARS-CoV infection or with cancer. ${ }^{479-482}$ Patients placed under Transmission Precautions may experience increased anxiety, depression, loneliness, and stress as well as anger and hostility. ${ }^{483,484}$ Compared with control patients, adult patients isolated for MRSA were more likely to have preventable adverse events (eg, falls, ulcers, or fluid and electrolyte abnormalities), to complain about their care, to have fewer vital signs taken, and to have more days without physician progress notes. ${ }^{485}$ Two systematic reviews similarly concluded that patients under Contact Precautions had less contact with healthcare personnel; decreased satisfaction with their care; more noninfectious adverse events, including decubitus ulcers and falls; delayed transfer to longterm care facilities; and more symptoms consistent with anxiety and depression. ${ }^{486,487}$ Inconsistent use of PPE by healthcare personnel or the time required to put on PPE was confusing and troubling to patients and increased their anxiety. ${ }^{479}$

Others found that patients under Contact Precautions were more likely to have symptoms of depression and anxiety at the time of admission but were not at increased risk of developing depression, anxiety, or negative moods during hospitalization. ${ }^{488}$ Similarly, personal attributes were associated with the development of depression and anxiety rather than the use of isolation precautions. ${ }^{479}$

Not all the psychosocial effects of isolation precautions are negative. Short-term infection prevention measures did not influence patients' level of anxiety, depression, or quality of life, but such patients had a positive attitude toward the precautions used. ${ }^{484}$ Some patients felt they had more freedom from ward routines and more control over their own activities. Some liked the privacy and quiet, particularly at night. ${ }^{479}$

Studies among people with CF. Parents and children with CF 10 years of age and older were surveyed about their center's segregation policy, whereby children with CF were provided single rooms when hospitalized and had to remain in their room throughout hospitalization. ${ }^{489}$ The majority agreed with the policy and understood that it was intended to maintain the children's health. Most parents felt that the health benefits outweighed the negative impacts, including social isolation. Parents worried about boredom, being able to keep their child in the hospital room, and the increased burden of entertaining their child. However, they also expressed relief at not having to worry about cross infection. The primary concerns expressed by the children were boredom and isolation. Factors that influenced the children's opinions were level of maturity, stage of development, and their experiences during previous admission(s). Following the spread of an epidemic clone of $P$. aeruginosa, the majority (85\%) of parents and children with CF 12 years of age and older (63\%) gave favorable responses for the need for cohort segregation, although negative responses were largely from the adolescent age group. ${ }^{400}$ 
Interventions to minimize the impact of isolation precautions. The CDC recommends that hospitals should anticipate and counteract possible anxiety, depression, perceptions of stigma, reduced contact with healthcare personnel, and other adverse events that may result from isolation precautions. ${ }^{5,6}$ Interventions to improve communication and physical facilities can ameliorate the negative effects; patient satisfaction was highest among isolated patients who were kept informed about their care ${ }^{491}$ Providing both written and individualized information with improved communication from staff can increase satisfaction and positive emotions. ${ }^{492}$

Increasing psychological support is also helpful. Investigators emphasize that patients in isolation require frequent contact with other people, including visitors and healthcare personnel, to prevent boredom and loneliness. ${ }^{492-494}$ Communication can be enhanced by human touch and humor displayed by healthcare personnel, especially by nurses who generally spend more time at the bedside.

Parents of children with CF emphasized that providing play therapy services, televisions, video games and movies, toys, crayons, and structured daily activities (eg, physiotherapy, school, pet therapy, and exercise) can reduce the impact of isolation. ${ }^{489,492,494,495}$ Children suggested that access to the Internet, mobile phones, and interactive resources and being able to leave their rooms would make isolation more tolerable. ${ }^{489}$ Physical facilities can also be altered to decrease the impact of isolation. Familiar items from home, such as pictures or personal belongings, can decrease the impact of isolation, as can providing patients with windows that view the ward or the outdoors. ${ }^{492,494}$ Possible strategies to minimize the adverse psychosocial impact of isolation precautions are provided in Table 11.

Online social networking. Online social networking can provide an opportunity for adults and children with CF to communicate with each other about personal issues and to give and receive valuable peer support outside the healthcare setting. ${ }^{496}$ CFfone has been developed to provide an intervention to improve adherence in adolescents with $\mathrm{CF}$ via a web-enabled cell phone that provides CF information and social support. ${ }^{497}$ However, CF caregivers should recognize both the power and the potential risks of these tools, should face-to-face meetings result from the communication initiated by online resources.

Thus, while IP\&C practices serve to protect people with $\mathrm{CF}$ from acquiring or spreading pathogens, awareness of the potential adverse effects of isolation should prompt the CF care team, people with CF, and their families to implement strategies designed to alleviate negative effects. This could improve adherence to IP\&C practices and improve the quality of healthcare encounters.

\section{Challenges to Implementation of IP\&C ReCOMMENDATIONS}

Challenges experienced by healthcare personnel. Potential challenges to implementation of healthcare guidelines include knowledge, attitude, and practice barriers. ${ }^{498}$ Relevant challenges and potential solutions to enhance implementation of the IP\&C in CF guideline are displayed in Tables $12^{498,499}$ and 13. Several barriers to implementation of the 2003 Infection Control Guideline for CF were identified among healthcare personnel $(n=528)$. These included lack of awareness of the guidelines $(40 \%)$, lack of familiarity with the recommendation to discourage socialization among hospitalized people with CF (31\%), disagreement with the recommendation to discourage socialization (32\%), and lack of confidence (selfefficacy) that the respondent could discourage socialization $(48 \%){ }^{499}$ Lack of self-efficacy was strongly associated with poor adherence to the recommendation to educate people with CF to perform hand hygiene and to disinfect their nebulizers. Others have similarly reported that recommendations that require counseling and education of patients are associated with a lack of self-efficacy by providers. ${ }^{498}$

Most respondents (84\%) caring for individuals with CF believed that implementation of the guideline would improve the health outcomes of their patients. ${ }^{499}$ Access to a copy of

TA B LE 11. Possible Strategies to Minimize the Adverse Psychosocial Impact of Isolation Precautions among People with Cystic Fibrosis (CF)

Incorporate people with $\mathrm{CF}$ and their families into discussion of daily plan of care

Encourage visits from individuals without $\mathrm{CF}$

Provide additional activities to help children pass the time (eg, art supplies, board and card games)

Provide television, DVDs, and video games

Consider animal-assisted therapy

Enlist visits from child life staff or volunteers

Provide computer and e-mail access

Provide written and individualized information about the need for isolation precautions

Increase psychological support

Bring familiar items from home

Allow to leave room (if feasible) accompanied by a trained staff member at least once daily

Arrange single-patient use of play room with cleaning after individual leaves

Provide daily schedule of medically related interventions

Ensure consistent communication with healthcare personnel 
TA B Le 12. Knowledge, Attitudes, and Practice Barriers Related to Implementing the Infection Prevention and Control Guideline in Cystic Fibrosis (CF)

\begin{tabular}{|c|c|c|}
\hline Category, paradigm & Barrier & Potential solutions \\
\hline $\begin{array}{l}\text { Lack of awareness or } \\
\text { familiarity }\end{array}$ & $\begin{array}{l}\text { No knowledge of infection prevention and } \\
\text { control guideline or no familiarity with } \\
\text { specific recommendations }\end{array}$ & $\begin{array}{l}\text { Easy access to guidelines } \\
\text { Review recommendations with CF care team and inpa- } \\
\text { tient staff }\end{array}$ \\
\hline Lack of education & $\begin{array}{l}\text { No provision of education to healthcare } \\
\text { personnel or to people with CF or } \\
\text { families }\end{array}$ & $\begin{array}{l}\text { Engage people with CF and their families } \\
\text { Develop easy-to-understand, eye-catching educational } \\
\text { handouts/brochures } \\
\text { Provide education and booster education in age- and } \\
\text { language-appropriate form }\end{array}$ \\
\hline \multicolumn{3}{|l|}{ Attitudes } \\
\hline Lack of agreement & $\begin{array}{l}\text { Disagreement with specific } \\
\text { recommendations }\end{array}$ & Review evidence, provide rationale \\
\hline Lack of outcome expectancy & $\begin{array}{l}\text { Do not believe recommended practice can } \\
\text { improve health outcomes }\end{array}$ & $\begin{array}{l}\text { Track center-specific and national trends to link adher- } \\
\text { ence to outcomes and share with staff, patients, and } \\
\text { families }\end{array}$ \\
\hline Inertia of current practice & $\begin{array}{l}\text { Believe recommendations are ineffective or } \\
\text { not applicable and reluctant to change } \\
\text { familiar practices }\end{array}$ & $\begin{array}{l}\text { Share quality improvement initiatives and successful in- } \\
\text { terventions among CF centers } \\
\text { Recruit early adaptors, positive deviants }\end{array}$ \\
\hline \multicolumn{3}{|l|}{ Practices } \\
\hline Lack of resources & $\begin{array}{l}\text { Lack of time, money, personnel, space, } \\
\text { supplies, equipment, and/or administra- } \\
\text { tive support } \\
\text { Belief that practices are inconvenient, } \\
\text { time-consuming, costly }\end{array}$ & $\begin{array}{l}\text { Engage with infection prevention and control teams } \\
\text { Provide adequate supplies and equipment at point of } \\
\text { care } \\
\text { Seek administrative support } \\
\text { Use return demonstrations } \\
\text { Perform quality improvement initiatives and report out- } \\
\text { comes to staff, families and administrators } \\
\text { Anticipate and monitor for unintended consequences }\end{array}$ \\
\hline
\end{tabular}

NOTE. Modeled after Cabana et $\mathrm{al}^{498}$ and Garber et al. ${ }^{499}$

the 2003 Infection Control Guideline for CF was associated with increased agreement with the recommendations and increased self-efficacy. Notably, physicians were more likely to have a copy of the guideline than other members of the CF care team. Interventions to overcome the lack of self-efficacy could include didactic lectures, practical skills workshops and training, and sharing best practices by early adopters of the recommendations (Section IV.B)..$^{500}$

Challenges experienced by people with CF and their families. Potential challenges related to implementation of the 2003 Infection Control Guideline for CF experienced by people with CF and the parents of children with CF were also explored. ${ }^{381}$ Among 1,399 respondents, 65\% were aware of the guideline. Of those aware, 34\% reported that they had never discussed the guideline with their CF care team, and only $30 \%$ reported that they had discussed the guideline more than once. More than 1 discussion with the CF care team was associated with increased knowledge, self-efficacy, and outcome expectancy. This suggests that booster or enhanced education could reduce barriers to implementation of IP\&C practices. While $83 \% \mathrm{knew}$ that germs could be transmitted person to person, only $64 \%$ and $59 \%$ knew that people with CF should avoid close contact even when not coughing or in the CF clinic, respectively. Most respondents were advised to perform hand hygiene $(80 \%)$, to avoid close contact with others with CF (70\%), and to clean their nebulizers (90\%). However, fewer were educated about specific practices, such as performing hand hygiene when entering (39\%) or leaving $(49 \%)$ the CF clinic or cleaning their nebulizer after each use $(69 \%)$. Few respondents believed that their health outcomes could be improved by avoiding close contact during hospitalization $(30 \%)$ or in the CF clinic (32\%). These findings 
тA в LE 13. Strategies to Enhance Implementation of the Infection Prevention and Control Guideline among Healthcare Personnel, People with Cystic Fibrosis (CF), and Families of People with CF

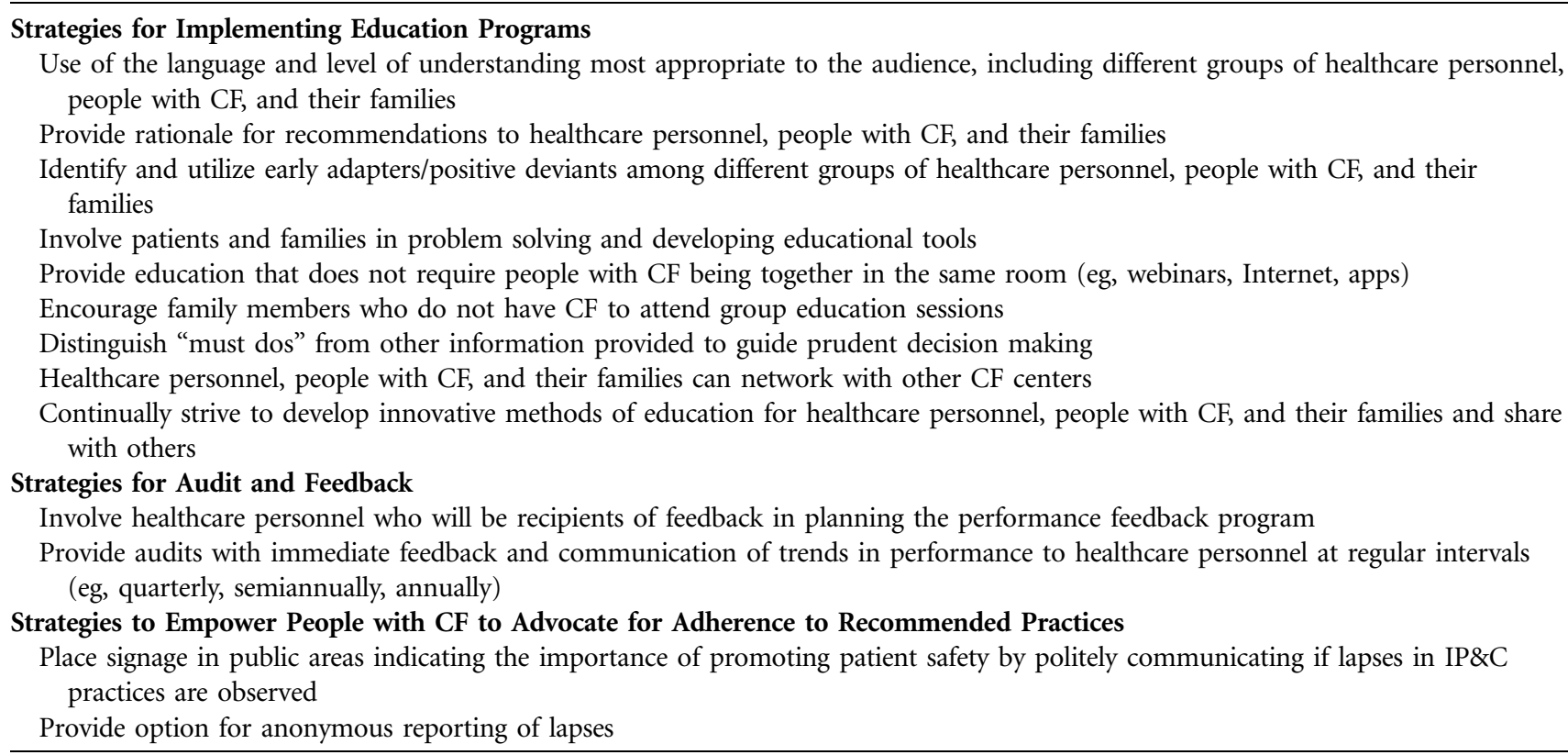

provide insights into specific educational content for individuals with $\mathrm{CF}$ and their families. Enhanced educational materials designed specifically for people with CF and their families exist (http://www.cff.org) but should be expanded by individual centers as needed.

\section{RESEARCH AGEN DA}

\section{SCV S. AUREUS}

1. The role of SCV S. aureus (and SCVs of other species, eg, $P$. aeruginosa) should continue to be studied to provide evidence for the need to standardize the processing of $\mathrm{CF}$ specimens to look for this phenotype.

\section{Transmission of CF Pathogens}

2. Future studies should continue to address the frequency of shared strains of CF pathogens, including P. aeruginosa and NTM. This could potentially be accomplished using reference laboratories, such as have been developed for B. cepacia complex.

3. The routes of transmission of $M$. abscessus, including the potential for transmission by droplet nuclei, should be further studied.

4. The role played by specific niches in the natural environment (eg, natural bodies of water or soil) in the transmission of CF pathogens should continue to be studied.

\section{Defining Best IP\&C Practices}

5. Additional studies are needed to describe the implementation and impact of effective IP\&C practices in CF cen- ters without epidemic clones or in CF centers that reduced transmission during a recognized outbreak. These should include epidemiologic studies, observational studies, and ethnographic research.

6. Additional studies should be performed to determine the efficacy of cohort segregation based on pathogen status versus all-patient separation.

7. Vaccination rates, particularly for influenza, obtained for people with CF and healthcare personnel could be used as patient safety and quality measures at CF centers.

8. Criteria should be developed to define a person with $\mathrm{CF}$ who had previously been culture positive for a specific pathogen and is now culture negative for that pathogen to be free of that pathogen.

\section{BARRIERS TO IMPLEMENTATION}

9. The CF community is strongly encouraged to engage the IP\&C community in discussions to find strategies to implement these recommendations and to overcome barriers to implementation.

10. Additional studies are required to understand the differences between the perceptions of healthcare personnel and people with CF and families regarding outcome expectancy following implementation of IP\&C practices.

\section{Healthcare Personnel with CF}

11. In 2003, healthcare personnel with CF were identified as a group that would benefit from further research. Ten years later, that need remains. 


\section{COMMITTEE MEMBERS (ALPHABETICAL OR DER )}

Rebekah F. Brown, MD

Department of Pediatrics, Vanderbilt University Medical Center, Nashville, Tennessee

Elizabeth A. Bryson, RN, MSN, PPCN-BC, CS

Department of Pediatrics, Akron Children's Hospital, Akron, Ohio

Mary Jo Chambers, LCSW, MSW

Department of Social Work, Arkansas Children's Hospital, Little Rock, Arkansas

Veronica S. Downer, RN

University of Michigan Hospital, Ann Arbor, Michigan

Jill Fliege, APRN

Pulmonary Division, Nebraska Medical Center, Omaha, Nebraska

Leslie A. Hazle, MS, RN, CPN, CPHQ

Current affiliation: Director, Clinical Operations and Performance Improvement, Spectrum Health Medical Group, Grand Rapids, Michigan

Manu Jain, MD, MS

Department of Medicine, Feinberg School of Medicine, Northwestern University, Chicago, Illinois

John J. LiPuma, MD

Department of Pediatrics and Communicable Diseases, University of Michigan Medical School, Ann Arbor, Michigan

Department of Epidemiology, University of Michigan School of Public Health, Ann Arbor, Michigan

Bruce C. Marshall, MD, MMM

Medical Department, Cystic Fibrosis Foundation, Bethesda, Maryland

Catherine O'Malley, RRT-NPS, AS

Department of Pediatrics, Ann and Robert H. Lurie Children's Hospital of Chicago, Chicago, Illinois

Suzanne R. Pattee, JD

Adult with cystic fibrosis, Silver Spring, Maryland

Gail Potter-Bynoe, BS

Department of Infection Prevention and Control, Boston Children's Hospital, Boston, Massachusetts

Siobhan Reid

Parent
Karen A. Robinson, $\mathrm{PhD}$

Johns Hopkins University, Baltimore, Maryland

Kathryn A. Sabadosa, MPH

Dartmouth Institute for Health Policy and Clinical Practice, Geisel School of Medicine at Dartmouth, Lebanon, New Hampshire

Lisa Saiman, MD, MPH

Department of Pediatrics, Columbia University Medical Center, New York, New York

Department of Infection Prevention and Control, NewYorkPresbyterian Hospital, New York, New York

H. Joel Schmidt, MD

Department of Pediatrics, Children's Hospital of Richmond at Virginia Commonwealth University, Richmond, Virginia

Jane D. Siegel, MD

Department of Pediatrics, University of Texas Southwestern Medical Center, Dallas, Texas

Children's Medical Center, Dallas, Texas

Elizabeth Tullis, MD, FRCPC

Department of Medicine, University of Toronto, and Keenan Research Centre of Li Ka Shing Knowledge Institute, St. Michael's Hospital, Toronto, Canada

Jennifer Webber

Parent

David J. Weber, MD, MPH

Departments of Medicine and Pediatrics, University of North Carolina at Chapel Hill Medical School, Chapel Hill, North Carolina

\section{ACKNOWLEDGMENTS}

Financial support. The Cystic Fibrosis Foundation provided the publication costs for this document.

Potential conflicts of interest. None of the authors have financial support or conflicts of interest to disclose relevant to this guideline.

Address correspondence to Lisa Saiman, MD, MPH, Columbia University, 622 West 168th Street, PH4 West Room 470, New York, NY 10032 (ls5@ cumc.columbia.edu).

The Cystic Fibrosis Foundation is located at 6931 Arlington Road, Bethesda, MD 20804; phone: 1-800-FIGHTCF; website: http://www.cff.org.

Presented in part: 26th Annual North American Cystic Fibrosis Conference; Orlando, Florida; October 2012; and 27th Annual North American Cystic Fibrosis Conference; Salt Lake City, Utah; October 2013.

\section{REF E R E N C E S}

1. Saiman L, Siegel J. Infection control recommendations for patients with cystic fibrosis: microbiology, important pathogens, 
and infection control practices to prevent patient-to-patient transmission. Infect Control Hosp Epidemiol 2003;24:S6-S52.

2. Sehulster L, Chinn RY. Guidelines for environmental infection control in health-care facilities: recommendations of CDC and the Healthcare Infection Control Practices Advisory Committee (HICPAC). MMWR Recomm Rep 2003;52:1-42.

3. Tablan OC, Anderson LJ, Besser R, Bridges C, Hajjeh R. Guidelines for preventing health-care-associated pneumonia, 2003: recommendations of CDC and the Healthcare Infection Control Practices Advisory Committee. MMWR Recomm Rep 2004; 53:1-36.

4. Jensen PA, Lambert LA, Iademarco MF, Ridzon R. Guidelines for preventing the transmission of Mycobacterium tuberculosis in health-care settings, 2005. MMWR Recomm Rep 2005;54:1141.

5. Siegel JD, Rhinehart E, Jackson M, Chiarello L. Management of multidrug-resistant organisms in health care settings, 2006. Am J Infect Control 2007;35:S165-S193.

6. Siegel JD, Rhinehart E, Jackson M, Chiarello L. 2007 Guideline for isolation precautions: preventing transmission of infectious agents in health care settings. Am J Infect Control 2007;35:S65S164.

7. Rutala WA, Weber DJ; Healthcare Infection Control Practices Advisory Committee (HICPAC). Guideline for Disinfection and Sterilization in Healthcare Facilities, 2008. http://www.cdc.gov /hicpac/pdf/guidelines/disinfection_nov_2008.pdf. Published 2008. Accessed January 2014.

8. Calfee DP, Salgado CD, Classen D, et al. Strategies to prevent transmission of methicillin-resistant Staphylococcus aureus in acute care hospitals. Infect Control Hosp Epidemiol 2008; 29(suppl 1):S62-S80.

9. World Health Organization. Guidelines on Hand Hygiene in Healthcare. http://whqlibdoc.who.int/publications/2009 /9789241597906_eng.pdf. Published 2009. Accessed January 13, 2014.

10. Division of Healthcare Quality Promotion, National Center for Emerging and Zoonotic Infectious Diseases, Centers for Disease Control and Prevention. Infection Prevention Checklist for Outpatient Settings: Minimum Expectations for Safe Care. http:// www.cdc.gov/HAI/pdfs/guidelines/ambulatory-care-checklist -07-2011.pdf. Published 2011. Accessed January 2014.

11. Advisory Committee on Immunization Practices; Centers for Disease Control and Prevention. Immunization of health-care personnel: recommendations of the Advisory Committee on Immunization Practices (ACIP). MMWR Recomm Rep 2011; 60:1-45.

12. Guzman-Cottrill JA, Ravin KA, Bryant KA, Zerr DM, Kociolek L, Siegel JD. Infection prevention and control in residential facilities for pediatric patients and their families. Infect Control Hosp Epidemiol 2013;34:1003-1041.

13. Rubin LG, Levin MJ, Ljungman P, et al. 2013 IDSA clinical practice guideline for vaccination of the immunocompromised host. Clin Infect Dis 2014;58:309-318.

14. Centers for Disease Control and Prevention. Seasonal Influenza (Flu). http://www.cdc.gov/flu/. Published 2014. Accessed January $13,2014$.

15. Glatman-Freedman A, Portelli I, Jacobs SK, et al. Attack rates assessment of the 2009 pandemic H1N1 influenza A in children and their contacts: a systematic review and meta-analysis. PLoS ONE 2012;7:e50228.
16. Dantes R, Mu Y, Belflower R, et al. National burden of invasive methicillin-resistant Staphylococcus aureus infections, United States, 2011. JAMA Intern Med 2013;173:1970-1978.

17. Iwamoto $\mathrm{M}, \mathrm{Mu} \mathrm{Y}$, Lynfield $\mathrm{R}$, et al. Trends in invasive methicillin-resistant Staphylococcus aureus infections. Pediatrics 2013;132:e817-e824.

18. Sievert DM, Ricks P, Edwards JR, et al. Antimicrobial-resistant pathogens associated with healthcare-associated infections: summary of data reported to the National Healthcare Safety Network at the Centers for Disease Control and Prevention, 2009-2010. Infect Control Hosp Epidemiol 2013;34:1-14.

19. Savard P, Perl TM. A call for action: managing the emergence of multidrug-resistant Enterobacteriaceae in the acute care settings. Curr Opin Infect Dis 2012;25:371-377.

20. Coia JE, Duckworth GJ, Edwards DI, et al. Guidelines for the control and prevention of meticillin-resistant Staphylococcus aureus (MRSA) in healthcare facilities. J Hosp Infect 2006; 63(suppl 1):S1-S44.

21. Cystic Fibrosis Foundation. National Patient Registry 2012 Annual Data Report. Bethesda, MD: Cystic Fibrosis Foundation, 2013.

22. Dasenbrook EC, Merlo CA, Diener-West M, Lechtzin N, Boyle MP. Persistent methicillin-resistant Staphylococcus aureus and rate of $\mathrm{FEV}_{1}$ decline in cystic fibrosis. Am J Respir Crit Care Med 2008;178:814-821.

23. Dasenbrook EC, Checkley W, Merlo CA, Konstan MW, Lechtzin N, Boyle MP. Association between respiratory tract methicillin-resistant Staphylococcus aureus and survival in cystic fibrosis. JAMA 2010;303:2386-2392.

24. Leung JM, Olivier KN. Nontuberculous mycobacteria in patients with cystic fibrosis. Semin Respir Crit Care Med 2013;34: 124-134.

25. Kalish LA, Waltz DA, Dovey M, et al. Impact of Burkholderia dolosa on lung function and survival in cystic fibrosis. Am J Respir Crit Care Med 2006;173:421-425.

26. Lipuma JJ. The changing microbial epidemiology in cystic fibrosis. Clin Microbiol Rev 2010;23:299-323.

27. Fothergill JL, Walshaw MJ, Winstanley C. Transmissible strains of Pseudomonas aeruginosa in cystic fibrosis lung infections. Eur Respir J 2012;40:227-238.

28. Aaron SD, Vandemheen KL, Ramotar K, et al. Infection with transmissible strains of Pseudomonas aeruginosa and clinical outcomes in adults with cystic fibrosis. JAMA 2010;304:21452153.

29. Waters V, Zlosnik JE, Yau YC, Speert DP, Aaron SD, Guttman DS. Comparison of three typing methods for Pseudomonas aeruginosa isolates from patients with cystic fibrosis. Eur J Clin Microbiol Infect Dis 2012;31:3341-3350.

30. Luna RA, Millecker LA, Webb CR, et al. Molecular epidemiological surveillance of multidrug-resistant Pseudomonas aeruginosa isolates in a pediatric population of patients with cystic fibrosis and determination of risk factors for infection with the Houston-1 strain. J Clin Microbiol 2013;51:1237-1240.

31. Elborn JS. Fixing cystic fibrosis CFTR with correctors and potentiators: off to a good start. Thorax 2012;67:4-5.

32. Robinson KA, Saldanha IJ, McKoy NA. Development of a framework to identify research gaps from systematic reviews. J Clin Epidemiol 2011;64:1325-1330.

33. Petitti DB, Teutsch SM, Barton MB, Sawaya GF, Ockene JK, DeWitt T. Update on the methods of the U.S. Preventive Ser- 
vices Task Force: insufficient evidence. Ann Intern Med 2009; 150:199-205.

34. Sawaya GF, Guirguis-Blake J, LeFevre M, Harris R, Petitti D. Update on the methods of the U.S. Preventive Services Task Force: estimating certainty and magnitude of net benefit. Ann Intern Med 2007;147:871-875.

35. Guyatt GH, Oxman AD, Vist GE, et al. GRADE: an emerging consensus on rating quality of evidence and strength of recommendations. BMJ 2008;336:924-926.

36. Ahmed F, Temte JL, Campos-Outcalt D, Schunemann HJ. Methods for developing evidence-based recommendations by the Advisory Committee on Immunization Practices (ACIP) of the U.S. Centers for Disease Control and Prevention (CDC). Vaccine 2011;29:9171-9176.

37. Umscheid CA, Agarwal RK, Brennan PJ. Updating the guideline development methodology of the Healthcare Infection Control Practices Advisory Committee (HICPAC). Am J Infect Control 2010;38:264-273.

38. Griffin MR. Influenza vaccination of healthcare workers: making the grade for action. Clin Infect Dis 2014;58:58-60.

39. Miller MB, Gilligan PH. Laboratory aspects of management of chronic pulmonary infections in patients with cystic fibrosis. J Clin Microbiol 2003;41:4009-4015.

40. UK Cystic Fibrosis Trust. Laboratory Standards for Processing Microbiological Samples from People with Cystic Fibrosis. https://www.cysticfibrosis.org.uk/media/82034/cd-laboratory -standards-sept10.pdf. Published 2010. Accessed January 13, 2014.

41. Zhou J, Garber E, Desai M, Saiman L. Compliance of clinical microbiology laboratories in the United States with current recommendations for processing respiratory tract specimens from patients with cystic fibrosis. J Clin Microbiol 2006;44: $1547-1549$

42. Rosenfeld M, Emerson J, Accurso F, et al. Diagnostic accuracy of oropharyngeal cultures in infants and young children with cystic fibrosis. Pediatr Pulmonol 1999;28:321-328.

43. Al-Saleh S, Dell SD, Grasemann H, et al. Sputum induction in routine clinical care of children with cystic fibrosis. J Pediatr 2010;157:1006.e1-1011.e1.

44. Razvi S, Quittell L, Sewall A, Quinton H, Marshall B, Saiman L. Respiratory microbiology of patients with cystic fibrosis in the United States, 1995 to 2005. Chest 2009;136:1554-1560.

45. Davidson AG, Chilvers MA, Lillquist YP. Effects of a Pseudomonas aeruginosa eradication policy in a cystic fibrosis clinic. Curr Opin Pulm Med 2012;18:615-621.

46. Alby K, Gilligan PH, Miller MB. Comparison of matrix-assisted laser desorption ionization-time of flight (MALDI-TOF) mass spectrometry platforms for the identification of gram-negative rods from patients with cystic fibrosis. J Clin Microbiol 2013; 51:3852-3854.

47. Desai AP, Stanley T, Atuan M, et al. Use of matrix assisted laser desorption ionisation-time of flight mass spectrometry in a paediatric clinical laboratory for identification of bacteria commonly isolated from cystic fibrosis patients. J Clin Pathol 2012; 65:835-838.

48. Fernandez-Olmos A, Garcia-Castillo M, Morosini MI, Lamas A, Maiz L, Canton R. MALDI-TOF MS improves routine identification of non-fermenting gram negative isolates from cystic fibrosis patients. J Cyst Fibros 2012;11:59-62.

49. Hurley MN, Ariff AH, Bertenshaw C, Bhatt J, Smyth AR. Re- sults of antibiotic susceptibility testing do not influence clinical outcome in children with cystic fibrosis. J Cyst Fibros 2012;11: 288-292.

50. Macdonald D, Cuthbertson L, Doherty C, et al. Early Pseudomonas aeruginosa infection in individuals with cystic fibrosis: is susceptibility testing justified? J Antimicrob Chemother 2010; 65:2373-2375.

51. Cheng K, Smyth RL, Govan JR, et al. Spread of $\beta$-lactamresistant Pseudomonas aeruginosa in a cystic fibrosis clinic. Lancet 1996;348:639-642.

52. Denton M, Kerr K, Mooney L, et al. Transmission of colistinresistant Pseudomonas aeruginosa between patients attending a pediatric cystic fibrosis center. Pediatr Pulmonol 2002;34:257261.

53. Jones AM, Govan JR, Doherty CJ, et al. Spread of a multiresistant strain of Pseudomonas aeruginosa in an adult cystic fibrosis clinic. Lancet 2001;358:557-558.

54. Lynch SV, Bruce KD. The cystic fibrosis airway microbiome. Cold Spring Harb Perspect Med 2013;3:a009738.

55. Rabin HR, Surette MG. The cystic fibrosis airway microbiome. Curr Opin Pulm Med 2012;18:622-627.

56. Zemanick ET, Sagel SD, Harris JK. The airway microbiome in cystic fibrosis and implications for treatment. Curr Opin Pediatr 2011;23:319-324.

57. Zhao J, Schloss PD, Kalikin LM, et al. Decade-long bacterial community dynamics in cystic fibrosis airways. Proc Natl Acad Sci USA 2012;109:5809-5814.

58. Lipuma JJ. Molecular tools for epidemiologic study of infectious diseases. Pediatr Infect Dis J 1998;17:667-675.

59. Morel AS, Saiman L. The role of molecular epidemiologic typing in pediatric infection control. Semin Pediatr Infect Dis 2001; 12:100-106.

60. Tenover FC, Arbeit RD, Goering RV, et al. Interpreting chromosomal DNA restriction patterns produced by pulsed-field gel electrophoresis: criteria for bacterial strain typing. J Clin Microbiol 1995;33:2233-2239.

61. Mahenthiralingam E, Campbell ME, Foster J, Lam JS, Speert DP. Random amplified polymorphic DNA typing of Pseudomonas aeruginosa isolates recovered from patients with cystic fibrosis. J Clin Microbiol 1996;34:1129-1135.

62. van Belkum A, Sluijuter M, de Groot R, Verbrugh H, Hermans PW. Novel BOX repeat PCR assay for high-resolution typing of Streptococcus pneumoniae strains. J Clin Microbiol 1996;34: 1176-1179.

63. Urwin R, Maiden MC. Multi-locus sequence typing: a tool for global epidemiology. Trends Microbiol 2003;11:479-487.

64. Multi Locus Sequence Typing website. http://www.mlst.net. Published 2014. Accessed January 14, 2014.

65. Snitkin ES, Zelazny AM, Thomas PJ, et al. Tracking a hospital outbreak of carbapenem-resistant Klebsiella pneumoniae with whole-genome sequencing. Sci Transl Med 2012;4:148ra116.

66. Bryant JM, Grogono DM, Greaves D, et al. Whole-genome sequencing to identify transmission of Mycobacterium abscessus between patients with cystic fibrosis: a retrospective cohort study. Lancet 2013;381:1551-1560.

67. International Burkholderia cepacia Working Group website. http://users.ugent.be/ tcoenye/index_bestanden/index.htm. Published 2010. Accessed January 13, 2014.

68. Cystic Fibrosis Foundation. National Patient Registry 2012 An- 
nual Data Report to the Center Directors. Bethesda, MD: Cystic Fibrosis Foundation, 2013.

69. Burkholder W. Sour skin: a bacterial rot of onion bulbs. Phytopathology 1950;40:115-117.

70. Coenye T, Mahenthiralingam E, Henry D, et al. Burkholderia ambifaria sp. nov., a novel member of the Burkholderia cepacia complex including biocontrol and cystic fibrosis-related isolates. Int J Syst Evol Microbiol 2001;51:1481-1490.

71. Vandamme P, Henry D, Coenye T, et al. Burkholderia anthina sp. nov. and Burkholderia pyrrocinia, two additional Burkholderia cepacia complex bacteria, may confound results of new molecular diagnostic tools. FEMS Immunol Med Microbiol 2002;33:143-149.

72. Vanlaere E, Baldwin A, Gevers D, et al. Taxon K, a complex within the Burkholderia cepacia complex, comprises at least two novel species, Burkholderia contaminans sp. nov. and Burkholderia lata sp. nov. Int J Syst Evol Microbiol 2009;59:102-111.

73. Vandamme P, Holmes B, Coenye T, et al. Burkholderia cenocepacia sp. nov.- a new twist to an old story. Res Microbiol 2003;154:91-96.

74. Vandamme P, Holmes B, Vancanneyt M, et al. Occurrence of multiple genomovars of Burkholderia cepacia in cystic fibrosis patients and proposal of Burkholderia multivorans sp. nov. Int J Syst Bacteriol 1997;47:1188-1200.

75. Vandamme P, Mahenthiralingam E, Holmes B, et al. Identification and population structure of Burkholderia stabilis sp. nov. (formerly Burkholderia cepacia genomovar IV). J Clin Microbiol 2000;38:1042-1047.

76. Vanlaere E, Lipuma JJ, Baldwin A, et al. Burkholderia latens sp. nov., Burkholderia diffusa sp. nov., Burkholderia arboris sp. nov., Burkholderia seminalis sp. nov. and Burkholderia metallica sp. nov., novel species within the Burkholderia cepacia complex. Int J Syst Evol Microbiol 2008;58:1580-1590.

77. Yabuuchi E, Kawamura Y, Ezaki T, et al. Burkholderia uboniae sp. nov., L-arabinose-assimilating but different from Burkholderia thailandensis and Burkholderia vietnamiensis. Microbiol Immunol 2000;44:307-317.

78. Peeters C, Zlosnik JE, Spilker T, Hird TJ, Lipuma JJ, Vandamme P. Burkholderia pseudomultivorans sp. nov., a novel Burkholderia cepacia complex species from human respiratory samples and the rhizosphere. Syst Appl Microbiol 2013;36:483-489.

79. Vermis K, Coenye T, LiPuma JJ, Mahenthiralingam E, Nelis HJ, Vandamme P. Proposal to accommodate Burkholderia cepacia genomovar VI as Burkholderia dolosa sp. nov. Int J Syst Evol Microbiol 2004;54:689-691.

80. Gillis M, Tran Van V, Bardin R, et al. Polyphasic taxonomy in the genus Burkholderia leading to an emended description of the genus and proposition of Burkholderia vietnamiensis sp. nov. for $\mathrm{N}_{2}$-fixing isolates from rice in Vietnam. Int $J$ Syst Bacteriol 1995;45:274.

81. Lipuma JJ. Update on the Burkholderia cepacia complex. Curr Opin Pulm Med 2005;11:528-533.

82. Whitby PW, Pope LC, Carter KB, LiPuma JJ, Stull TL. Speciesspecific PCR as a tool for the identification of Burkholderia gladioli. J Clin Microbiol 2000;38:282-285.

83. Ciofu O, Hansen CR, Hoiby N. Respiratory bacterial infections in cystic fibrosis. Curr Opin Pulm Med 2013;19:251-258.

84. Prunier AL, Malbruny B, Laurans M, Brouard J, Duhamel JF, Leclercq R. High rate of macrolide resistance in Staphylococcus aureus strains from patients with cystic fibrosis reveals high proportions of hypermutable strains. J Infect Dis 2003;187: 1709-1716.

85. Besier S, Zander J, Kahl BC, Kraiczy P, Brade V, Wichelhaus TA. The thymidine-dependent small-colony-variant phenotype is associated with hypermutability and antibiotic resistance in clinical Staphylococcus aureus isolates. Antimicrob Agents Chemother 2008;52:2183-2189.

86. Proctor RA, von Eiff C, Kahl BC, et al. Small colony variants: a pathogenic form of bacteria that facilitates persistent and recurrent infections. Nat Rev Microbiol 2006;4:295-305.

87. Proctor RA, Kahl B, von Eiff C, Vaudaux PE, Lew DP, Peters G. Staphylococcal small colony variants have novel mechanisms for antibiotic resistance. Clin Infect Dis 1998;27(suppl 1):S68-S74.

88. Sadowska B, Bonar A, von Eiff C, et al. Characteristics of Staphylococcus aureus, isolated from airways of cystic fibrosis patients, and their small colony variants. FEMS Immunol Med Microbiol 2002;32:191-197.

89. Mitchell G, Grondin G, Bilodeau G, Cantin AM, Malouin F. Infection of polarized airway epithelial cells by normal and small-colony variant strains of Staphylococcus aureus is increased in cells with abnormal cystic fibrosis transmembrane conductance regulator function and is influenced by NF- $\kappa \mathrm{B}$. Infect Immun 2011;79:3541-3551.

90. Proctor RA, van Langevelde P, Kristjansson M, Maslow JN, Arbeit RD. Persistent and relapsing infections associated with small-colony variants of Staphylococcus aureus. Clin Infect Dis 1995;20:95-102.

91. Kahl BC, Duebbers A, Lubritz G, et al. Population dynamics of persistent Staphylococcus aureus isolated from the airways of cystic fibrosis patients during a 6-year prospective study. $J$ Clin Microbiol 2003;41:4424-4427.

92. Besier S, Smaczny C, von Mallinckrodt C, et al. Prevalence and clinical significance of Staphylococcus aureus small-colony variants in cystic fibrosis lung disease. J Clin Microbiol 2007;45: $168-172$.

93. Schneider M, Muhlemann K, Droz S, Couzinet S, Casaulta C, Zimmerli S. Clinical characteristics associated with isolation of small-colony variants of Staphylococcus aureus and Pseudomonas aeruginosa from respiratory secretions of patients with cystic fibrosis. J Clin Microbiol 2008;46:1832-1834.

94. Hoffman LR, Deziel E, D’Argenio DA, et al. Selection for Staphylococcus aureus small-colony variants due to growth in the presence of Pseudomonas aeruginosa. Proc Natl Acad Sci USA 2006;103:19890-19895.

95. Wolter DJ, Emerson JC, McNamara S, et al. Staphylococcus aureus small-colony variants are independently associated with worse lung disease in children with cystic fibrosis. Clin Infect Dis 2013;57:384-391.

96. Kirisits MJ, Prost L, Starkey M, Parsek MR. Characterization of colony morphology variants isolated from Pseudomonas aeruginosa biofilms. Appl Environ Microbiol 2005;71:48094821 .

97. Anderson SW, Stapp JR, Burns JL, Qin X. Characterization of small-colony-variant Stenotrophomonas maltophilia isolated from the sputum specimens of five patients with cystic fibrosis. J Clin Microbiol 2007;45:529-535.

98. Haussler S, Lehmann C, Breselge C, et al. Fatal outcome of lung transplantation in cystic fibrosis patients due to small- 
colony variants of the Burkholderia cepacia complex. Eur J Clin Microbiol Infect Dis 2003;22:249-253.

99. Kasperbauer SH, Daley CL. Diagnosis and treatment of infections due to Mycobacterium avium complex. Semin Respir Crit Care Med 2008;29:569-576.

100. Adekambi T, Berger P, Raoult D, Drancourt M. rpoB gene sequence-based characterization of emerging nontuberculous mycobacteria with descriptions of Mycobacterium bolletii sp. nov., Mycobacterium phocaicum sp. nov. and Mycobacterium aubagnense sp. nov. Int J Syst Evol Microbiol 2006;56:133-143.

101. Adekambi T, Reynaud-Gaubert M, Greub G, et al. Amoebal coculture of "Mycobacterium massiliense" sp. nov. from the sputum of a patient with hemoptoic pneumonia. J Clin $\mathrm{Mi}$ crobiol 2004;42:5493-5501.

102. Olivier KN, Weber DJ, Wallace RJ Jr, et al. Nontuberculous mycobacteria. I. Multicenter prevalence study in cystic fibrosis. Am J Respir Crit Care Med 2003;167:828-834.

103. Levy I, Grisaru-Soen G, Lerner-Geva L, et al. Multicenter crosssectional study of nontuberculous mycobacterial infections among cystic fibrosis patients, Israel. Emerg Infect Dis 2008;14: 378-384.

104. Roux AL, Catherinot E, Ripoll F, et al. Multicenter study of prevalence of nontuberculous mycobacteria in patients with cystic fibrosis in France. J Clin Microbiol 2009;47:4124-4128.

105. Pihet M, Carrere J, Cimon B, et al. Occurrence and relevance of filamentous fungi in respiratory secretions of patients with cystic fibrosis-a review. Med Mycol 2009;47:387-397.

106. Bains SN, Judson MA. Allergic bronchopulmonary aspergillosis. Clin Chest Med 2012;33:265-281.

107. Knutsen AP, Slavin RG. Allergic bronchopulmonary aspergillosis in asthma and cystic fibrosis. Clin Dev Immunol 2011; 2011:843763.

108. Kousha M, Tadi R, Soubani AO. Pulmonary aspergillosis: a clinical review. Eur Respir Rev 2011;20:156-174.

109. Moss RB. Allergic bronchopulmonary aspergillosis and Aspergillus infection in cystic fibrosis. Curr Opin Pulm Med 2010; 16:598-603.

110. Liu JC, Modha DE, Gaillard EA. What is the clinical significance of filamentous fungi positive sputum cultures in patients with cystic fibrosis? J Cyst Fibros 2013;12:187-193.

111. Braun AT, Merlo CA. Cystic fibrosis lung transplantation. Curr Opin Pulm Med 2011;17:467-472.

112. Hota B. Contamination, disinfection, and cross-colonization: are hospital surfaces reservoirs for nosocomial infection? Clin Infect Dis 2004;39:1182-1189.

113. Dowell SF, Simmerman JM, Erdman DD, et al. Severe acute respiratory syndrome coronavirus on hospital surfaces. Clin Infect Dis 2004;39:652-657.

114. Bischoff WE, Swett K, Leng I, Peters TR. Exposure to influenza virus aerosols during routine patient care. J Infect Dis 2013; 207:1037-1046.

115. Advisory Committee on Immunization Practices. Prevention and control of influenza with vaccines: interim recommendations of the Advisory Committee on Immunization Practices (ACIP), 2013. MMWR Morb Mortal Wkly Rep 2013;62:356.

116. Tran K, Cimon K, Severn M, Pessoa-Silva CL, Conly J. Aerosol generating procedures and risk of transmission of acute respiratory infections to healthcare workers: a systematic review. PLOS ONE 2012;7:e35797.

117. Roy CJ, Milton DK. Airborne transmission of communicable infection-the elusive pathway. N Engl J Med 2004;350:17101712.

118. Wong TW, Lee CK, Tam W, et al. Cluster of SARS among medical students exposed to single patient, Hong Kong. Emerg Infect Dis 2004;10:269-276.

119. Clifton IJ, Fletcher LA, Beggs CB, Denton M, Conway SP, Peckham DG. An aerobiological model of aerosol survival of different strains of Pseudomonas aeruginosa isolated from people with cystic fibrosis. J Cyst Fibros 2010;9:64-68.

120. Festini F, Taccetti G, Galici V, et al. A 1-m distance is not safe for children with cystic fibrosis at risk for cross-infection with Pseudomonas aeruginosa. Am J Infect Control 2010;38:244-245.

121. Wainwright CE, France MW, O’Rourke P, et al. Cough-generated aerosols of Pseudomonas aeruginosa and other gramnegative bacteria from patients with cystic fibrosis. Thorax 2009;64:926-931.

122. Clifton IJ, Peckham DG. Defining routes of airborne transmission of Pseudomonas aeruginosa in people with cystic fibrosis. Expert Rev Respir Med 2010;4:519-529.

123. Spicknall IH, Koopman JS, Nicas M, Pujol JM, Li S, Eisenberg JN. Informing optimal environmental influenza interventions: how the host, agent, and environment alter dominant routes of transmission. PLoS Comput Biol 2010;6:e1000969.

124. Brankston G, Gitterman L, Hirji Z, Lemieux C, Gardam M. Transmission of influenza A in human beings. Lancet Infect Dis 2007;7:257-265.

125. Zuckerman JB, Prato BS, Clock S, et al. Characterizing bacterial air contamination during CF outpatient visits. Pediatr Pulmonol 2012;47(suppl 35):326.

126. Ferroni A, Werkhauser-Bertrand A, Le Bourgeois M, et al. Bacterial contamination in the environment of hospitalised children with cystic fibrosis. J Cyst Fibros 2008;7:477-482.

127. Panagea S, Winstanley C, Walshaw MJ, Ledson MJ, Hart CA. Environmental contamination with an epidemic strain of Pseudomonas aeruginosa in a Liverpool cystic fibrosis centre, and study of its survival on dry surfaces. J Hosp Infect 2005;59:102107.

128. Zuckerman JB, Zuaro DE, Prato BS, et al. Bacterial contamination of cystic fibrosis clinics. J Cyst Fibros 2009;8:186-192.

129. Aitken ML, Limaye A, Pottinger P, et al. Respiratory outbreak of Mycobacterium abscessus subspecies massiliense in a lung transplant and cystic fibrosis center. Am J Respir Crit Care Med 2012;185:231-232.

130. Speert DP, Campbell ME, Henry DA, et al. Epidemiology of Pseudomonas aeruginosa in cystic fibrosis in British Columbia, Canada. Am J Respir Crit Care Med 2002;166:988-993.

131. Jelsbak L, Johansen HK, Frost AL, et al. Molecular epidemiology and dynamics of Pseudomonas aeruginosa populations in lungs of cystic fibrosis patients. Infect Immun 2007;75:22142224.

132. Spilker T, LiPuma JJ. Genotype analysis of Pseudomonas aeruginosa isolates from U.S. CF centers. Pediatr Pulmonol 2013; 48(suppl 36):306.

133. Biddick R, Spilker T, Martin A, LiPuma JJ. Evidence of transmission of Burkholderia cepacia, Burkholderia multivorans and Burkholderia dolosa among persons with cystic fibrosis. FEMS Microbiol Lett 2003;228:57-62.

134. LiPuma JJ, Dasen SE, Nielson DW, Stern RC, Stull TL. Personto-person transmission of Pseudomonas cepacia between patients with cystic fibrosis. Lancet 1990;336:1094-1096. 
135. Govan JR, Brown PH, Maddison J, et al. Evidence for transmission of Pseudomonas cepacia by social contact in cystic fibrosis. Lancet 1993;342:15-19.

136. Schlichting C, Branger C, Fournier JM, et al. Typing of Staphylococcus aureus by pulsed-field gel electrophoresis, zymotyping, capsular typing, and phage typing: resolution of clonal relationships. J Clin Microbiol 1993;31:227-232.

137. Elizur A, Orscheln RC, Ferkol TW, Dunne WM Jr, Storch GA, Cannon CL. Transmission of Panton-Valentine leukocidinpositive Staphylococcus aureus between patients with cystic fibrosis. J Pediatr 2007;151:90-92.

138. Givney R, Vickery A, Holliday A, Pegler M, Benn R. Methicillin-resistant Staphylococcus aureus in a cystic fibrosis unit. $J$ Hosp Infect 1997;35:27-36.

139. Cocchi P, Cariani L, Favari F, et al. Molecular epidemiology of meticillin-resistant Staphylococcus aureus in Italian cystic fibrosis patients: a national overview. J Cyst Fibros 2011;10:407411.

140. Denton M, Todd NJ, Kerr KG, Hawkey PM, Littlewood JM. Molecular epidemiology of Stenotrophomonas maltophilia isolated from clinical specimens from patients with cystic fibrosis and associated environmental samples. J Clin Microbiol 1998; 36:1953-1958.

141. Marzuillo C, De Giusti M, Tufi D, et al. Molecular characterization of Stenotrophomonas maltophilia isolates from cystic fibrosis patients and the hospital environment. Infect Control Hosp Epidemiol 2009;30:753-758.

142. McPhail GL, VanDyke R, Renchel M, LiPuma JJ, Joseph PM. An update on clinical outcomes associated with a clonal strain of Achromobacter (Alcaligenes) xylosoxidans. Pediatr Pulmonol 2009;44(suppl 32):310.

143. Krzewinski JW, Nguyen CD, Foster JM, Burns JL. Use of random amplified polymorphic DNA PCR to examine epidemiology of Stenotrophomonas maltophilia and Achromobacter (Alcaligenes) xylosoxidans from patients with cystic fibrosis. $J$ Clin Microbiol 2001;39:3597-3602.

144. Van Daele S, Verhelst R, Claeys G, et al. Shared genotypes of Achromobacter xylosoxidans strains isolated from patients at a cystic fibrosis rehabilitation center. J Clin Microbiol 2005;43: 2998-3002.

145. Harris KA, Kenna DT, Blauwendraat C, et al. Molecular fingerprinting of Mycobacterium abscessus strains in a cohort of pediatric cystic fibrosis patients. J Clin Microbiol 2012;50:17581761.

146. Baldwin A, Mahenthiralingam E, Thickett KM, et al. Multilocus sequence typing scheme that provides both species and strain differentiation for the Burkholderia cepacia complex. J Clin Microbiol 2005;43:4665-4673.

147. Coenye T, LiPuma JJ. Multilocus restriction typing: a novel tool for studying global epidemiology of Burkholderia cepacia complex infection in cystic fibrosis. J Infect Dis 2002;185:14541462.

148. Dasen SE, LiPuma JJ, Kostman JR, Stull TL. Characterization of PCR-ribotyping for Burkholderia (Pseudomonas) cepacia. J Clin Microbiol 1994;32:2422-2424.

149. LiPuma JJ, Mortensen JE, Dasen SE, et al. Ribotype analysis of Pseudomonas cepacia from cystic fibrosis treatment centers. J Pediatr 1988;113:859-862.

150. Spilker T, Baldwin A, Bumford A, Dowson CG, Mahenthir- alingam E, LiPuma JJ. Expanded multilocus sequence typing for Burkholderia species. J Clin Microbiol 2009;47:2607-2610.

151. Pegues DA, Carson LA, Tablan OC, et al; Summer Camp Study Group. Acquisition of Pseudomonas cepacia at summer camps for patients with cystic fibrosis. J Pediatr 1994;124:694-702.

152. Johnson WM, Tyler SD, Rozee KR. Linkage analysis of geographic and clinical clusters in Pseudomonas cepacia infections by multilocus enzyme electrophoresis and ribotyping. J Clin Microbiol 1994;32:924-930.

153. Pitt TL, Kaufmann ME, Patel PS, Benge LC, Gaskin S, Livermore DM. Type characterisation and antibiotic susceptibility of Burkholderia (Pseudomonas) cepacia isolates from patients with cystic fibrosis in the United Kingdom and the Republic of Ireland. J Med Microbiol 1996;44:203-210.

154. Chen JS, Witzmann KA, Spilker T, Fink RJ, LiPuma JJ. Endemicity and inter-city spread of Burkholderia cepacia genomovar III in cystic fibrosis. J Pediatr 2001;139:643-649.

155. Coenye T, LiPuma JJ. Population structure analysis of Burkholderia cepacia genomovar III: varying degrees of genetic recombination characterize major clonal complexes. Microbiology 2003;149:77-88.

156. Kumar A, Dietrich S, Schneider W, et al. Genetic relatedness of Burkholderia (Pseudomonas) cepacia isolates from five cystic fibrosis centers in Michigan. Respir Med 1997;91:485-492.

157. Springman AC, Jacobs JL, Somvanshi VS, et al. Genetic diversity and multihost pathogenicity of clinical and environmental strains of Burkholderia cenocepacia. Appl Environ Microbiol 2009;75:5250-5260.

158. Drevinek P, Vosahlikova S, Cinek O, et al. Widespread clone of Burkholderia cenocepacia in cystic fibrosis patients in the Czech Republic. J Med Microbiol 2005;54:655-659.

159. Mahenthiralingam E, Vandamme P, Campbell ME, et al. Infection with Burkholderia cepacia complex genomovars in patients with cystic fibrosis: virulent transmissible strains of genomovar III can replace Burkholderia multivorans. Clin Infect Dis 2001;33:1469-1475.

160. Speert DP, Henry D, Vandamme P, Corey M, Mahenthiralingam E. Epidemiology of Burkholderia cepacia complex in patients with cystic fibrosis, Canada. Emerg Infect Dis 2002;8:181-187.

161. Campana S, Taccetti G, Ravenni N, et al. Transmission of Burkholderia cepacia complex: evidence for new epidemic clones infecting cystic fibrosis patients in Italy. J Clin Microbiol 2005; 43:5136-5142.

162. Manno G, Dalmastri C, Tabacchioni S, et al. Epidemiology and clinical course of Burkholderia cepacia complex infections, particularly those caused by different Burkholderia cenocepacia strains, among patients attending an Italian cystic fibrosis center. J Clin Microbiol 2004;42:1491-1497.

163. Drevinek P, Cinek O, Melter J, Langsadl L, Navesnakova Y, Vavrova V. Genomovar distribution of the Burkholderia cepacia complex differs significantly between Czech and Slovak patients with cystic fibrosis. J Med Microbiol 2003;52:603-604.

164. Segonds C, Heulin T, Marty N, Chabanon G. Differentiation of Burkholderia species by PCR-restriction fragment length polymorphism analysis of the $16 \mathrm{~S}$ rRNA gene and application to cystic fibrosis isolates. J Clin Microbiol 1999;37:2201-2208.

165. Whiteford ML, Wilkinson JD, McColl JH, et al. Outcome of Burkholderia (Pseudomonas) cepacia colonisation in children with cystic fibrosis following a hospital outbreak. Thorax 1995; 50:1194-1198. 
166. Govan JR, Brown AR, Jones AM. Evolving epidemiology of Pseudomonas aeruginosa and the Burkholderia cepacia complex in cystic fibrosis lung infection. Future Microbiol 2007;2:153164.

167. Mortensen JE, Fisher MC, LiPuma JJ. Recovery of Pseudomonas cepacia and other Pseudomonas species from the environment. Infect Control Hosp Epidemiol 1995;16:30-32.

168. Johansen HK, Kovesi TA, Koch C, Corey M, Hoiby N, Levison H. Pseudomonas aeruginosa and Burkholderia cepacia infection in cystic fibrosis patients treated in Toronto and Copenhagen. Pediatr Pulmonol 1998;26:89-96.

169. Corey M, Farewell V. Determinants of mortality from cystic fibrosis in Canada, 1970-1989. Am J Epidemiol 1996;143:10071017.

170. Rosenfeld M, Davis R, FitzSimmons S, Pepe M, Ramsey B. Gender gap in cystic fibrosis mortality. Am J Epidemiol 1997; 145:794-803.

171. Murray S, Charbeneau J, Marshall BC, LiPuma JJ. Impact of Burkholderia infection on lung transplantation in cystic fibrosis. Am J Respir Crit Care Med 2008;178:363-371.

172. De Soyza A, Morris K, McDowell A, et al. Prevalence and clonality of Burkholderia cepacia complex genomovars in UK patients with cystic fibrosis referred for lung transplantation. Thorax 2004;59:526-528.

173. Scott FW, Pitt TL. Identification and characterization of transmissible Pseudomonas aeruginosa strains in cystic fibrosis patients in England and Wales. J Med Microbiol 2004;53:609-615.

174. Romling U, Fiedler B, Bosshammer J, et al. Epidemiology of chronic Pseudomonas aeruginosa infections in cystic fibrosis. J Infect Dis 1994;170:1616-1621.

175. Tummler B, Koopmann U, Grothues D, Weissbrodt H, Steinkamp G, von der Hardt H. Nosocomial acquisition of Pseudomonas aeruginosa by cystic fibrosis patients. J Clin Microbiol 1991;29:1265-1267.

176. Wolz C, Kiosz G, Ogle JW, et al. Pseudomonas aeruginosa crosscolonization and persistence in patients with cystic fibrosis: use of a DNA probe. Epidemiol Infect 1989;102:205-214.

177. Grothues D, Koopmann U, von der Hardt H, Tummler B. Genome fingerprinting of Pseudomonas aeruginosa indicates colonization of cystic fibrosis siblings with closely related strains. J Clin Microbiol 1988;26:1973-1977.

178. Pedersen SS, Koch C, Hoiby N, Rosendal K. An epidemic spread of multiresistant Pseudomonas aeruginosa in a cystic fibrosis centre. J Antimicrob Chemother 1986;17:505-516.

179. O'Carroll MR, Syrmis MW, Wainwright CE, et al. Clonal strains of Pseudomonas aeruginosa in paediatric and adult cystic fibrosis units. Eur Respir J 2004;24:101-106.

180. Armstrong D, Bell S, Robinson M, et al. Evidence for spread of a clonal strain of Pseudomonas aeruginosa among cystic fibrosis clinics. J Clin Microbiol 2003;41:2266-2267.

181. Armstrong DS, Nixon GM, Carzino R, et al. Detection of a widespread clone of Pseudomonas aeruginosa in a pediatric cystic fibrosis clinic. Am J Respir Crit Care Med 2002;166:983987.

182. Syrmis MW, O'Carroll MR, Sloots TP, et al. Rapid genotyping of Pseudomonas aeruginosa isolates harboured by adult and paediatric patients with cystic fibrosis using repetitive-elementbased PCR assays. J Med Microbiol 2004;53:1089-1096.

183. Bradbury R, Champion A, Reid DW. Poor clinical outcomes associated with a multi-drug resistant clonal strain of Pseu- domonas aeruginosa in the Tasmanian cystic fibrosis population. Respirology 2008;13:886-892.

184. van Mansfeld R, Willems R, Brimicombe R, et al. Pseudomonas aeruginosa genotype prevalence in Dutch cystic fibrosis patients and age dependency of colonization by various $P$. aeruginosa sequence types. J Clin Microbiol 2009;47:4096-4101.

185. Griffiths AL, Jamsen K, Carlin JB, et al. Effects of segregation on an epidemic Pseudomonas aeruginosa strain in a cystic fibrosis clinic. Am J Respir Crit Care Med 2005;171:1020-1025.

186. Ashish A, Shaw M, Winstanley C, Ledson MJ, Walshaw MJ. Increasing resistance of the Liverpool epidemic strain (LES) of Pseudomonas aeruginosa (Psa) to antibiotics in cystic fibrosis (CF)—a cause for concern? J Cyst Fibros 2012;11:173-179.

187. Al-Aloul M, Crawley J, Winstanley C, Hart CA, Ledson MJ, Walshaw MJ. Increased morbidity associated with chronic infection by an epidemic Pseudomonas aeruginosa strain in CF patients. Thorax 2004;59:334-336.

188. Jones AM, Dodd ME, Doherty CJ, Govan JR, Webb AK. Increased treatment requirements of patients with cystic fibrosis who harbour a highly transmissible strain of Pseudomonas aeruginosa. Thorax 2002;57:924-925.

189. Ashish A, Shaw M, McShane J, Ledson MJ, Walshaw MJ. Health-related quality of life in cystic fibrosis patients infected with transmissible Pseudomonas aeruginosa strains: cohort study. JRSM Short Rep 2012;3:12.

190. Spilker T, Vandamme P, Lipuma JJ. Identification and distribution of Achromobacter species in cystic fibrosis. J Cyst Fibros 2013;12:298-301.

191. Vu-Thien H, Darbord JC, Moissenet D, et al. Investigation of an outbreak of wound infections due to Alcaligenes xylosoxidans transmitted by chlorhexidine in a burns unit. Eur J Clin Microbiol Infect Dis 1998;17:724-726.

192. Weitkamp JH, Tang YW, Haas DW, Midha NK, Crowe JE Jr. Recurrent Achromobacter xylosoxidans bacteremia associated with persistent lymph node infection in a patient with hyperimmunoglobulin M syndrome. Clin Infect Dis 2000;31:11831187.

193. Duggan JM, Goldstein SJ, Chenoweth CE, Kauffman CA, Bradley SF. Achromobacter xylosoxidans bacteremia: report of four cases and review of the literature. Clin Infect Dis 1996;23:569576.

194. Dunne WM Jr, Maisch S. Epidemiological investigation of infections due to Alcaligenes species in children and patients with cystic fibrosis: use of repetitive-element-sequence polymerase chain reaction. Clin Infect Dis 1995;20:836-841.

195. Vu-Thien H, Moissenet D, Valcin M, Dulot C, Tournier G, Garbarg-Chenon A. Molecular epidemiology of Burkholderia cepacia, Stenotrophomonas maltophilia, and Alcaligenes xylosoxidans in a cystic fibrosis center. Eur J Clin Microbiol Infect Dis 1996;15:876-879.

196. Kanellopoulou M, Pournaras S, Iglezos H, Skarmoutsou N, Papafrangas E, Maniatis AN. Persistent colonization of nine cystic fibrosis patients with an Achromobacter (Alcaligenes) $x y$ losoxidans clone. Eur J Clin Microbiol Infect Dis 2004;23:336339.

197. Coenye T, Spilker T, Reik R, Vandamme P, Lipuma JJ. Use of PCR analyses to define the distribution of Ralstonia species recovered from patients with cystic fibrosis. J Clin Microbiol 2005;43:3463-3466

198. Coenye T, Vandamme P, LiPuma JJ. Infection by Ralstonia 
species in cystic fibrosis patients: identification of $R$. pickettii and $R$. mannitolilytica by polymerase chain reaction. Emerg Infect Dis 2002;8:692-696.

199. Vandamme P, Coenye T. Taxonomy of the genus Cupriavidus: a tale of lost and found. Int J Syst Evol Microbiol 2004;54:22852289.

200. Vandamme P, Goris J, Coenye T, et al. Assignment of Centers for Disease Control group IVc-2 to the genus Ralstonia as Ralstonia paucula sp. nov. Int J Syst Bacteriol 1999;49(pt 2): 663-669.

201. Coenye T, Falsen E, Vancanneyt M, et al. Classification of Alcaligenes faecalis-like isolates from the environment and human clinical samples as Ralstonia gilardii sp. nov. Int J Syst Bacteriol 1999;49(pt 2):405-413.

202. Coenye T, Vandamme P, LiPuma JJ. Ralstonia respiraculi sp. nov., isolated from the respiratory tract of cystic fibrosis patients. Int J Syst Evol Microbiol 2003;53:1339-1342.

203. Chen WM, Laevens S, Lee TM, et al. Ralstonia taiwanensis sp. nov., isolated from root nodules of Mimosa species and sputum of a cystic fibrosis patient. Int J Syst Evol Microbiol 2001;51: 1729-1735.

204. LiPuma JJ. Burkholderia and emerging pathogens in cystic fibrosis. Semin Respir Crit Care Med 2003;24:681-692.

205. Coenye T, Falsen E, Hoste B, et al. Description of Pandoraea gen. nov. with Pandoraea apista sp. nov., Pandoraea pulmonicola sp. nov., Pandoraea pnomenusa sp. nov., Pandoraea sputorum sp. nov. and Pandoraea norimbergensis comb. nov. Int J Syst Evol Microbiol 2000;50(pt 2):887-899.

206. Jorgensen IM, Johansen HK, Frederiksen B, et al. Epidemic spread of Pandoraea apista, a new pathogen causing severe lung disease in cystic fibrosis patients. Pediatr Pulmonol 2003;36: $439-446$.

207. Gorwitz RJ, Kruszon-Moran D, McAllister SK, et al. Changes in the prevalence of nasal colonization with Staphylococcus aureus in the United States, 2001-2004. J Infect Dis 2008;197: 1226-1234.

208. Rosenfeld M, Bernardo-Ocampo C, Emerson J, Genatossio A, Burns J, Gibson R. Prevalence of cystic fibrosis pathogens in the oropharynx of healthy children and implications for cystic fibrosis care. J Cyst Fibros 2012;11:456-457.

209. Goerke C, Kraning K, Stern M, Doring G, Botzenhart K, Wolz C. Molecular epidemiology of community-acquired Staphylococcus aureus in families with and without cystic fibrosis patients. J Infect Dis 2000;181:984-989.

210. Perl TM, Cullen JJ, Wenzel RP, et al. Intranasal mupirocin to prevent postoperative Staphylococcus aureus infections. N Engl J Med 2002;346:1871-1877.

211. Perl TM, Roy MC. Postoperative wound infections: risk factors and role of Staphylococcus aureus nasal carriage. J Chemother 1995;7(suppl 3):29-35.

212. von Eiff C, Becker K, Machka K, Stammer H, Peters G. Nasal carriage as a source of Staphylococcus aureus bacteremia. N Engl J Med 2001;344:11-16.

213. Branger C, Gardye C, Lambert-Zechovsky N. Persistence of Staphylococcus aureus strains among cystic fibrosis patients over extended periods of time. J Med Microbiol 1996;45:294-301.

214. Wertheim HF, Vos MC, Ott A, et al. Risk and outcome of nosocomial Staphylococcus aureus bacteraemia in nasal carriers versus non-carriers. Lancet 2004;364:703-705.

215. Stone A, Quittell L, Zhou J, et al. Staphylococcus aureus nasal colonization among pediatric cystic fibrosis patients and their household contacts. Pediatr Infect Dis J 2009;28:895-899.

216. Ridder-Schaphorn S, Ratjen F, Dubbers A, et al. Nasal Staphylococcus aureus carriage is not a risk factor for lower-airway infection in young cystic fibrosis patients. J Clin Microbiol 2007; 45:2979-2984.

217. Nadesalingam K, Conway SP, Denton M. Risk factors for acquisition of methicillin-resistant Staphylococcus aureus (MRSA) by patients with cystic fibrosis. J Cyst Fibros 2005;4:49-52.

218. Glikman D, Siegel JD, David MZ, et al. Complex molecular epidemiology of methicillin-resistant Staphylococcus aureus isolates from children with cystic fibrosis in the era of epidemic community-associated methicillin-resistant $S$ aureus. Chest 2008;133:1381-1387.

219. Muhlebach MS, Miller M, LaVange LM, Mayhew G, Goodrich JS, Miller MB. Treatment intensity and characteristics of MRSA infection in CF. J Cyst Fibros 2011;10:201-206.

220. Champion EA, Miller MB, Popowitch EB, Hobbs MM, Saiman L, Muhlebach MS. Antimicrobial susceptibility and molecular typing of MRSA in cystic fibrosis. Pediatr Pulmonol 2014;49: 230-237.

221. Al-Zubeidi D, Hogan PG, Boyle M, Burnham CA, Fritz SA. Molecular epidemiology of methicillin-resistant Staphylococcus aureus isolated in serial cultures from the respiratory tract of children with cystic fibrosis. Pediatr Infect Dis J 2014;33:549553.

222. Harris SR, Cartwright EJ, Torok ME, et al. Whole-genome sequencing for analysis of an outbreak of meticillin-resistant Staphylococcus aureus: a descriptive study. Lancet Infect Dis 2013;13:130-136.

223. Koser CU, Holden MT, Ellington MJ, et al. Rapid wholegenome sequencing for investigation of a neonatal MRSA outbreak. N Engl J Med 2012;366:2267-2275.

224. Vanderhelst E, De Meirleir L, Verbanck S, Pierard D, Vincken $\mathrm{W}$, Malfroot A. Prevalence and impact on $\mathrm{FEV}_{1}$ decline of chronic methicillin-resistant Staphylococcus aureus (MRSA) colonization in patients with cystic fibrosis: a single-center, case control study of 165 patients. J Cyst Fibros 2012;11:2-7.

225. Sanders DB, Bittner RC, Rosenfeld M, Redding GJ, Goss CH. Pulmonary exacerbations are associated with subsequent $\mathrm{FEV}_{1}$ decline in both adults and children with cystic fibrosis. Pediatr Pulmonol 2011;46:393-400.

226. Sawicki GS, Rasouliyan L, Pasta DJ, et al. The impact of incident methicillin resistant Staphylococcus aureus detection on pulmonary function in cystic fibrosis. Pediatr Pulmonol 2008; 43:1117-1123.

227. Bange FC, Brown BA, Smaczny C, Wallace RJ Jr, Bottger EC. Lack of transmission of Mycobacterium abscessus among patients with cystic fibrosis attending a single clinic. Clin Infect Dis 2001;32:1648-1650.

228. Jonsson BE, Gilljam M, Lindblad A, Ridell M, Wold AE, Welinder-Olsson C. Molecular epidemiology of Mycobacterium abscessus, with focus on cystic fibrosis. J Clin Microbiol 2007;45: 1497-1504.

229. Tettelin H, Davidson RM, Agrawal S, et al. High-level relatedness among Mycobacterium abscessus subsp. massiliense strains from widely separated outbreaks. Emerg Infect Dis 2014; 20:364-371.

230. Gross J, Doan M, Yamada S, et al. Mycobacterium abcessus 
epidemic among cystic fibrosis patients [abstract]. Pediatr Pulmonol 2013;48(suppl 36):302.

231. Chalermskulrat W, Sood N, Neuringer IP, et al. Nontuberculous mycobacteria in end stage cystic fibrosis: implications for lung transplantation. Thorax 2006;61:507-513.

232. Orens JB, Estenne M, Arcasoy S, et al. International guidelines for the selection of lung transplant candidates: 2006 updatea consensus report from the Pulmonary Scientific Council of the International Society for Heart and Lung Transplantation. J Heart Lung Transplant 2006;25:745-755.

233. Huang HC, Weigt SS, Derhovanessian A, et al. Nontuberculous mycobacterium infection after lung transplantation is associated with increased mortality. J Heart Lung Transplant 2011; 30:790-798.

234. Esther CR Jr, Esserman DA, Gilligan P, Kerr A, Noone PG. Chronic Mycobacterium abscessus infection and lung function decline in cystic fibrosis. J Cyst Fibros 2010;9:117-123.

235. Barbier F, Andremont A, Wolff M, Bouadma L. Hospitalacquired pneumonia and ventilator-associated pneumonia: recent advances in epidemiology and management. Curr Opin Pulm Med 2013;19:216-228.

236. Branski LK, Al-Mousawi A, Rivero H, Jeschke MG, Sanford AP, Herndon DN. Emerging infections in burns. Surg Infect (Larchmt) 2009;10:389-397.

237. Pendleton JN, Gorman SP, Gilmore BF. Clinical relevance of the ESKAPE pathogens. Expert Rev Anti Infect Ther 2013;11: 297-308.

238. McCallum SJ, Gallagher MJ, Corkill JE, Hart CA, Ledson MJ, Walshaw MJ. Spread of an epidemic Pseudomonas aeruginosa strain from a patient with cystic fibrosis (CF) to non-CF relatives. Thorax 2002;57:559-560.

239. Dy ME, Nord JA, LaBombardi VJ, Germana J, Walker P. Lack of throat colonization with Burkholderia cepacia among cystic fibrosis healthcare workers. Infect Control Hosp Epidemiol 1999; 20:90.

240. Agochukwu NQ, Rastinehad AR, Richter LA, et al. Prostatic abscess in a pediatric patient with chronic granulomatous disease: report of a unique case and review of the literature. $J$ Pediatr Surg 2012;47:400-403.

241. Fishman JA. Infections in immunocompromised hosts and organ transplant recipients: essentials. Liver Transpl 2011; 17(suppl 3):S34-S37.

242. Holmes A, Nolan R, Taylor R, et al. An epidemic of Burkholderia cepacia transmitted between patients with and without cystic fibrosis. J Infect Dis 1999;179:1197-1205.

243. Lowy FD. Staphylococcus aureus infections. N Engl J Med 1998; 339:520-532.

244. Chambers HF. The changing epidemiology of Staphylococcus aureus? Emerg Infect Dis 2001;7:178-182.

245. Creech CB 2nd, Kernodle DS, Alsentzer A, Wilson C, Edwards $\mathrm{KM}$. Increasing rates of nasal carriage of methicillin-resistant Staphylococcus aureus in healthy children. Pediatr Infect Dis J 2005;24:617-621.

246. Kluytmans J, van Belkum A, Verbrugh H. Nasal carriage of Staphylococcus aureus: epidemiology, underlying mechanisms, and associated risks. Clin Microbiol Rev 1997;10:505-520.

247. Lautenbach E, Tolomeo P, Nachamkin I, Hu B, Zaoutis TE. The impact of household transmission on duration of outpatient colonization with methicillin-resistant Staphylococcus aureus. Epidemiol Infect 2010;138:683-685.
248. Nouwen JL, Ott A, Kluytmans-Vandenbergh MF, et al. Predicting the Staphylococcus aureus nasal carrier state: derivation and validation of a "culture rule." Clin Infect Dis 2004;39:806-811.

249. Gesualdo F, Bongiorno D, Rizzo C, et al. MRSA nasal colonization in children: prevalence meta-analysis, review of risk factors and molecular genetics. Pediatr Infect Dis J 2013;32: 479-485.

250. Albrich WC, Harbarth S. Health-care workers: source, vector, or victim of MRSA? Lancet Infect Dis 2008;8:289-301.

251. Hawkins G, Stewart S, Blatchford O, Reilly J. Should healthcare workers be screened routinely for meticillin-resistant Staphylococcus aureus? a review of the evidence. J Hosp Infect 2011; 77:285-289.

252. Grant PS, Charns LG, Rawot BW, Benedetti SG. Consideration to culture health care workers related to increased methicillinresistant Staphylococcus aureus activity in a neonatal intensive care unit. Am J Infect Control 2008;36:638-643.

253. Fritz SA, Garbutt J, Elward A, Shannon W, Storch GA. Prevalence of and risk factors for community-acquired methicillinresistant and methicillin-sensitive Staphylococcus aureus colonization in children seen in a practice-based research network. Pediatrics 2008;121:1090-1098.

254. Barrett TW, Moran GJ. Methicillin-resistant Staphylococcus aureus infections among competitive sports participants-Colorado, Indiana, Pennsylvania, and Los Angeles County, 20002003. Ann Emerg Med 2004;43:43-47.

255. Kazakova SV, Hageman JC, Matava M, et al. A clone of methicillin-resistant Staphylococcus aureus among professional football players. N Engl J Med 2005;352:468-475.

256. Creech CB, Saye E, McKenna BD, et al. One-year surveillance of methicillin-resistant Staphylococcus aureus nasal colonization and skin and soft tissue infections in collegiate athletes. Arch Pediatr Adolesc Med 2010;164:615-620.

257. Oller AR, Province L, Curless B. Staphylococcus aureus recovery from environmental and human locations in 2 collegiate athletic teams. J Athl Train 2010;45:222-229.

258. Roberts MC, Soge OO, No D, Helgeson SE, Meschke JS. Characterization of methicillin-resistant Staphylococcus aureus isolated from public surfaces on a university campus, student homes and local community. J Appl Microbiol 2011;110:15311537.

259. Begier EM, Frenette K, Barrett NL, et al. A high-morbidity outbreak of methicillin-resistant Staphylococcus aureus among players on a college football team, facilitated by cosmetic body shaving and turf burns. Clin Infect Dis 2004;39:1446-1453.

260. Nguyen DM, Mascola L, Brancoft E. Recurring methicillinresistant Staphylococcus aureus infections in a football team. Emerg Infect Dis 2005;11:526-532.

261. Archibald LK, Shapiro J, Pass A, Rand K, Southwick F. Methicillin-resistant Staphylococcus aureus infection in a college football team: risk factors outside the locker room and playing field. Infect Control Hosp Epidemiol 2008;29:450-453.

262. Romano R, Lu D, Holtom P. Outbreak of community-acquired methicillin-resistant Staphylococcus aureus skin infections among a collegiate football team. J Athl Train 2006;41:141-145.

263. Rihn JA, Posfay-Barbe K, Harner CD, et al. Communityacquired methicillin-resistant Staphylococcus aureus outbreak in a local high school football team: unsuccessful interventions. Pediatr Infect Dis J 2005;24:841-843.

264. Bowers AL, Huffman GR, Sennett BJ. Methicillin-resistant 
Staphylococcus aureus infections in collegiate football players. Med Sci Sports Exerc 2008;40:1362-1367.

265. Stevens MP, Bearman G, Rosato A, Edmond M. Communityacquired methicillin resistant Staphylococcus aureus in a women's collegiate basketball team. South Med J 2008;101: 1067-1068.

266. Lear A, McCord G, Peiffer J, Watkins RR, Parikh A, Warrington S. Incidence of Staphylococcus aureus nasal colonization and soft tissue infection among high school football players. $J \mathrm{Am}$ Board Fam Med 2011;24:429-435.

267. Lindenmayer JM, Schoenfeld S, O’Grady R, Carney JK. Methicillin-resistant Staphylococcus aureus in a high school wrestling team and the surrounding community. Arch Intern Med 1998; 158:895-899.

268. Centers for Disease Control and Prevention. Methicillin-resistant Staphylococcus aureus (MRSA) infections: prevention information and advice for athletes. http://www.cdc.gov /mrsa/groups/advice-for-athletes.html. Published 2010. Accessed October 21, 2013.

269. de Almeida MB, Zerbinati RM, Tateno AF, et al. Rhinovirus $\mathrm{C}$ and respiratory exacerbations in children with cystic fibrosis. Emerg Infect Dis 2010;16:996-999.

270. Smyth AR, Smyth RL, Tong CY, Hart CA, Heaf DP. Effect of respiratory virus infections including rhinovirus on clinical status in cystic fibrosis. Arch Dis Child 1995;73:117-120.

271. Wat D, Gelder C, Hibbitts S, et al. The role of respiratory viruses in cystic fibrosis. J Cyst Fibros 2008;7:320-328.

272. Hoek RA, Paats MS, Pas SD, et al. Incidence of viral respiratory pathogens causing exacerbations in adult cystic fibrosis patients. Scand J Infect Dis 2013;45:65-69.

273. Ortiz JR, Neuzil KM, Victor JC, Wald A, Aitken ML, Goss CH. Influenza-associated cystic fibrosis pulmonary exacerbations. Chest 2010;137:852-860.

274. Asner S, Waters V, Solomon M, et al. Role of respiratory viruses in pulmonary exacerbations in children with cystic fibrosis. $J$ Cyst Fibros 2012;11:433-439.

275. Poritz MA, Blaschke AJ, Byington CL, et al. FilmArray, an automated nested multiplex PCR system for multi-pathogen detection: development and application to respiratory tract infection. PLoS ONE 2011;6:e26047.

276. National Center for Emerging and Zoonotic Infectious Diseases, Centers for Disease Control and Prevention. http:// www.cdc.gov/ncezid. Published 2013. Accessed January 13, 2014.

277. Maeda Y, Stanley T, Stirling J, et al. No evidence of transmission of bacteria between reptiles and a CF patient-a case report of a young adult CF patient and reptiles. Zoonoses Public Health 2010;57:e47-e53.

278. Ner Z, Ross LA, Horn MV, et al. Bordetella bronchiseptica infection in pediatric lung transplant recipients. Pediatr Transplant 2003;7:413-417.

279. Hemsworth S, Pizer B. Pet ownership in immunocompromised children-a review of the literature and survey of existing guidelines. Eur J Oncol Nurs 2006;10:117-127.

280. Register KB, Sukumar N, Palavecino EL, Rubin BK, Deora R. Bordetella bronchiseptica in a paediatric cystic fibrosis patient: possible transmission from a household cat. Zoonoses Public Health 2012;59:246-250.

281. Mohan K, Fothergill JL, Storrar J, Ledson MJ, Winstanley C, Walshaw MJ. Transmission of Pseudomonas aeruginosa epi- demic strain from a patient with cystic fibrosis to a pet cat. Thorax 2008;63:839-840.

282. Loeffler A, Lloyd DH. Companion animals: a reservoir for methicillin-resistant Staphylococcus aureus in the community? Epidemiol Infect 2010;138:595-605.

283. Ferreira JP, Fowler VG Jr, Correa MT, Lyman R, Ruffin F, Anderson KL. Transmission of methicillin-resistant Staphylococcus aureus between human and hamster. J Clin Microbiol 2011;49:1679-1680.

284. Lewis FM, Marsh BJ, von Reyn CF. Fish tank exposure and cutaneous infections due to Mycobacterium marinum: tuberculin skin testing, treatment, and prevention. Clin Infect Dis 2003;37:390-397.

285. Pandian TK, Deziel PJ, Otley CC, Eid AJ, Razonable RR. Mycobacterium marinum infections in transplant recipients: case report and review of the literature. Transpl Infect Dis 2008;10: 358-363.

286. Centers for Disease Control and Prevention. Healthy Pets Healthy People. http://www.cdc.gov/healthypets/. Published 2013. Accessed January 13, 2014.

287. Lefebvre SL, Golab GC, Christensen E, et al. Guidelines for animal-assisted interventions in health care facilities. Am J Infect Control 2008;36:78-85.

288. Curran KA, Miller J. Guidelines for animal-assisted interventions in health care facilities. Am J Infect Control 2009;37:257258.

289. US Department of Justice Civil Rights Division. Commonly Asked Questions about Service Animals in Places of Business. http://www.ada.gov/qasrvc.htm. Published 2008. Accessed January 13, 2014.

290. Sheahan T, Rockx B, Donaldson E, et al. Mechanisms of zoonotic severe acute respiratory syndrome coronavirus host range expansion in human airway epithelium. J Virol 2008;82:22742285.

291. Moore JE, Goldsmith CE, Millar BC, et al. Cystic fibrosis and the isolation of Pseudomonas aeruginosa from horses. Vet Rec 2008;163:399-400.

292. Chowdhury P, Heinemann JA. The general secretory pathway of Burkholderia gladioli pv. agaricicola BG164R is necessary for cavity disease in white button mushrooms. Appl Environ Microbiol 2006;72:3558-3565.

293. Fiore A, Laevens S, Bevivino A, et al. Burkholderia cepacia complex: distribution of genomovars among isolates from the maize rhizosphere in Italy. Environ Microbiol 2001;3:137-143.

294. Miller SC, LiPuma JJ, Parke JL. Culture-based and non-growthdependent detection of the Burkholderia cepacia complex in soil environments. Appl Environ Microbiol 2002;68:3750-3758.

295. Ramette A, LiPuma JJ, Tiedje JM. Species abundance and diversity of Burkholderia cepacia complex in the environment. Appl Environ Microbiol 2005;71:1193-1201.

296. Vermis K, Brachkova M, Vandamme P, Nelis H. Isolation of Burkholderia cepacia complex genomovars from waters. Syst Appl Microbiol 2003;26:595-600.

297. Muhdi K, Edenborough FP, Gumery L, et al. Outcome for patients colonised with Burkholderia cepacia in a Birmingham adult cystic fibrosis clinic and the end of an epidemic. Thorax 1996;51:374-377.

298. Coenye T, Spilker T, Van Schoor A, LiPuma JJ, Vandamme P. Recovery of Burkholderia cenocepacia strain PHDC from cystic fibrosis patients in Europe. Thorax 2004;59:952-954. 
299. Fisher MC, LiPuma JJ, Dasen SE, et al. Source of Pseudomonas cepacia: ribotyping of isolates from patients and from the environment. J Pediatr 1993;123:745-747.

300. LiPuma JJ, Spilker T, Coenye T, Gonzalez CF. An epidemic Burkholderia cepacia complex strain identified in soil. Lancet 2002;359:2002-2003.

301. Baldwin A, Mahenthiralingam E, Drevinek P, et al. Environmental Burkholderia cepacia complex isolates in human infections. Emerg Infect Dis 2007;13:458-461.

302. Mahenthiralingam E, Baldwin A, Dowson CG. Burkholderia cepacia complex bacteria: opportunistic pathogens with important natural biology. J Appl Microbiol 2008;104:1539-1551.

303. McNeely D, Moore JE, Elborn JS, Millar BC, Rendall J, Dooley JS. Isolation of Burkholderia cenocepacia and Burkholderia vietnamiensis from human sewage. Int J Environ Health Res 2009; 19:157-162.

304. Remold SK, Brown CK, Farris JE, Hundley TC, Perpich JA, Purdy ME. Differential habitat use and niche partitioning by Pseudomonas species in human homes. Microb Ecol 2011;62: 505-517.

305. Regnath T, Kreutzberger M, Illing S, Oehme R, Liesenfeld O. Prevalence of Pseudomonas aeruginosa in households of patients with cystic fibrosis. Int J Hyg Environ Health 2004;207: 585-588.

306. Schelstraete P, Van Daele S, De Boeck K, et al. Pseudomonas aeruginosa in the home environment of newly infected cystic fibrosis patients. Eur Respir J 2008;31:822-829.

307. Barben J, Hafen G, Schmid J. Pseudomonas aeruginosa in public swimming pools and bathroom water of patients with cystic fibrosis. J Cyst Fibros 2005;4:227-231.

308. Rosenfeld M, Emerson J, McNamara S, et al. Risk factors for age at initial Pseudomonas acquisition in the cystic fibrosis epic observational cohort. J Cyst Fibros 2012;11:446-453.

309. Romling U, Wingender J, Muller H, Tummler B. A major Pseudomonas aeruginosa clone common to patients and aquatic habitats. Appl Environ Microbiol 1994;60:1734-1738.

310. Hauben L, Vauterin L, Moore ER, Hoste B, Swings J. Genomic diversity of the genus Stenotrophomonas. Int J Syst Bacteriol 1999;49(pt 4):1749-1760.

311. Spencer RC. The emergence of epidemic, multiple-antibioticresistant Stenotrophomonas (Xanthomonas) maltophilia and Burkholderia (Pseudomonas) cepacia. J Hosp Infect 1995; 30(suppl):453-464.

312. Kay SE, Clark RA, White KL, Peel MM. Recurrent Achromobacter piechaudii bacteremia in a patient with hematological malignancy. J Clin Microbiol 2001;39:808-810.

313. Falkinham JO 3rd. Surrounded by mycobacteria: nontuberculous mycobacteria in the human environment. J Appl Microbiol 2009;107:356-367.

314. De Groote MA, Pace NR, Fulton K, Falkinham JO 3rd. Relationships between Mycobacterium isolates from patients with pulmonary mycobacterial infection and potting soils. Appl Environ Microbiol 2006;72:7602-7606.

315. Fujita K, Ito Y, Hirai T, et al. Genetic relatedness of Mycobacterium avium-intracellulare complex isolates from patients with pulmonary MAC disease and their residential soils. Clin Microbiol Infect 2013;19:537-541.

316. Decker BK, Palmore TN. The role of water in healthcareassociated infections. Curr Opin Infect Dis 2013;26:345-351.

317. Festini F, Taccetti G, Mannini C, et al. Patient risk of contact with respiratory pathogens from inanimate surfaces in a cystic fibrosis outpatient clinic: a prospective study over a four-year period. Pediatr Pulmonol 2007;42:779-784.

318. Klausner JD, Zukerman C, Limaye AP, Corey L. Outbreak of Stenotrophomonas maltophilia bacteremia among patients undergoing bone marrow transplantation: association with faulty replacement of handwashing soap. Infect Control Hosp Epidemiol 1999;20:756-758.

319. Weber DJ, Rutala WA, Blanchet CN, Jordan M, Gergen MF. Faucet aerators: a source of patient colonization with Stenotrophomonas maltophilia. Am J Infect Control 1999;27:59-63.

320. Denton M, Rajgopal A, Mooney L, et al. Stenotrophomonas maltophilia contamination of nebulizers used to deliver aerosolized therapy to inpatients with cystic fibrosis. J Hosp Infect 2003;55:180-183.

321. Valdezate S, Vindel A, Martin-Davila P, Del Saz BS, Baquero F, Canton R. High genetic diversity among Stenotrophomonas maltophilia strains despite their originating at a single hospital. J Clin Microbiol 2004;42:693-699.

322. Donskey CJ. Does improving surface cleaning and disinfection reduce health care-associated infections? Am J Infect Control 2013;41:S12-S19.

323. Harris AD. How important is the environment in the emergence of nosocomial antimicrobial-resistant bacteria? Clin Infect Dis 2008;46:686-688.

324. Otter JA, Yezli S, French GL. The role played by contaminated surfaces in the transmission of nosocomial pathogens. Infect Control Hosp Epidemiol 2011;32:687-699.

325. Weber DJ, Rutala WA, Miller MB, Huslage K, Sickbert-Bennett E. Role of hospital surfaces in the transmission of emerging health care-associated pathogens: norovirus, Clostridium difficile, and Acinetobacter species. Am J Infect Control 2010;38: S25-S33.

326. Hota B, Blom DW, Lyle EA, Weinstein RA, Hayden MK. Interventional evaluation of environmental contamination by vancomycin-resistant enterococci: failure of personnel, product, or procedure? J Hosp Infect 2009;71:123-131.

327. Boyce JM, Havill NL, Otter JA, Adams NM. Widespread environmental contamination associated with patients with diarrhea and methicillin-resistant Staphylococcus aureus colonization of the gastrointestinal tract. Infect Control Hosp Epidemiol 2007;28:1142-1147.

328. Edmond MB, Wenzel RP, Pasculle AW. Vancomycin-resistant Staphylococcus aureus: perspectives on measures needed for control. Ann Intern Med 1996;124:329-334.

329. Weber DJ, Anderson D, Rutala WA. The role of the surface environment in healthcare-associated infections. Curr Opin Infect Dis 2013;26:338-344.

330. Vajravelu RK, Guerrero DM, Jury LA, Donskey CJ. Evaluation of stethoscopes as vectors of Clostridium difficile and methicillin-resistant Staphylococcus aureus. Infect Control Hosp Epidemiol 2012;33:96-98.

331. Blydt-Hansen T, Subbarao K, Quennec P, McDonald J. Recovery of respiratory syncytial virus from stethoscopes by conventional viral culture and polymerase chain reaction. Pediatr Infect Dis J 1999;18:164-165.

332. Kerr JR, Martin H, Chadwick MV, Edwards A, Hodson ME, Geddes DM. Evidence against transmission of Pseudomonas aeruginosa by hands and stethoscopes in a cystic fibrosis unit. J Hosp Infect 2002;50:324-326. 
333. Muscarella LF. Prevention of disease transmission during flexible laryngoscopy. Am J Infect Control 2007;35:536-544.

334. Muscarella LF. Recommendations to resolve inconsistent guidelines for the reprocessing of sheathed and unsheathed rigid laryngoscopes. Infect Control Hosp Epidemiol 2007;28: 504-507.

335. Bou R, Aguilar A, Perpinan J, et al. Nosocomial outbreak of Pseudomonas aeruginosa infections related to a flexible bronchoscope. J Hosp Infect 2006;64:129-135.

336. DiazGranados CA, Jones MY, Kongphet-Tran T, et al. Outbreak of Pseudomonas aeruginosa infection associated with contamination of a flexible bronchoscope. Infect Control Hosp Epidemiol 2009;30:550-555.

337. Kirschke DL, Jones TF, Craig AS, et al. Pseudomonas aeruginosa and Serratia marcescens contamination associated with a manufacturing defect in bronchoscopes. N Engl J Med 2003;348: 214-220.

338. Shimono N, Takuma T, Tsuchimochi N, et al. An outbreak of Pseudomonas aeruginosa infections following thoracic surgeries occurring via the contamination of bronchoscopes and an automatic endoscope reprocessor. I Infect Chemother 2008;14: 418-423.

339. Phillips MS, von Reyn CF. Nosocomial infections due to nontuberculous mycobacteria. Clin Infect Dis 2001;33:1363-1374.

340. Begg N, O’Mahony M, Penny P, Richardson EA. Mycobacterium chelonei associated with a hospital hydrotherapy pool. Community Med 1986;8:348-350.

341. Jones AM, Govan JR, Doherty CJ, et al. Identification of airborne dissemination of epidemic multiresistant strains of Pseudomonas aeruginosa at a CF centre during a cross infection outbreak. Thorax 2003;58:525-527.

342. Jimenez L. Microbial diversity in pharmaceutical product recalls and environments. PDA J Pharm Sci Technol 2007;61:383399.

343. Torbeck L, Raccasi D, Guilfoyle DE, Friedman RL, Hussong D. Burkholderia cepacia: this decision is overdue. PDA J Pharm Sci Technol 2011;65:535-543.

344. Weber DJ, Rutala WA, Sickbert-Bennett EE. Outbreaks associated with contaminated antiseptics and disinfectants. Antimicrob Agents Chemother 2007;51:4217-4224.

345. Balkhy HH, Cunningham G, Francis C, et al. A National Guard outbreak of Burkholderia cepacia infection and colonization secondary to intrinsic contamination of albuterol nebulization solution. Am J Infect Control 2005;33:182-188.

346. Berthelot P, Grattard F, Mahul P, et al. Ventilator temperature sensors: an unusual source of Pseudomonas cepacia in nosocomial infection. J Hosp Infect 1993;25:33-43.

347. Burdge DR, Nakielna EM, Noble MA. Case-control and vector studies of nosocomial acquisition of Pseudomonas cepacia in adult patients with cystic fibrosis. Infect Control Hosp Epidemiol 1993;14:127-130.

348. Estivariz CF, Bhatti LI, Pati R, et al. An outbreak of Burkholderia cepacia associated with contamination of albuterol and nasal spray. Chest 2006;130:1346-1353.

349. Hamill RJ, Houston ED, Georghiou PR, et al. An outbreak of Burkholderia (formerly Pseudomonas) cepacia respiratory tract colonization and infection associated with nebulized albuterol therapy. Ann Intern Med 1995;122:762-766.

350. Hutchinson J, Runge W, Mulvey M, et al. Burkholderia cepacia infections associated with intrinsically contaminated ultra- sound gel: the role of microbial degradation of parabens. Infect Control Hosp Epidemiol 2004;25:291-296.

351. Kutty PK, Moody B, Gullion JS, et al. Multistate outbreak of Burkholderia cenocepacia colonization and infection associated with the use of intrinsically contaminated alcohol-free mouthwash. Chest 2007;132:1825-1831.

352. Loukil C, Saizou C, Doit C, et al. Epidemiologic investigation of Burkholderia cepacia acquisition in two pediatric intensive care units. Infect Control Hosp Epidemiol 2003;24:707-710.

353. Molina-Cabrillana J, Bolanos-Rivero M, Alvarez-Leon EE, et al. Intrinsically contaminated alcohol-free mouthwash implicated in a nosocomial outbreak of Burkholderia cepacia colonization and infection. Infect Control Hosp Epidemiol 2006;27: 1281-1282.

354. Nasser RM, Rahi AC, Haddad MF, Daoud Z, Irani-Hakime N, Almawi WY. Outbreak of Burkholderia cepacia bacteremia traced to contaminated hospital water used for dilution of an alcohol skin antiseptic. Infect Control Hosp Epidemiol 2004;25: 231-239.

355. Panlilio AL, Beck-Sague CM, Siegel JD, et al. Infections and pseudoinfections due to povidone-iodine solution contaminated with Pseudomonas cepacia. Clin Infect Dis 1992;14:10781083.

356. Pegues CF, Pegues DA, Ford DS, et al. Burkholderia cepacia respiratory tract acquisition: epidemiology and molecular characterization of a large nosocomial outbreak. Epidemiol Infect 1996;116:309-317.

357. Ramsey AH, Skonieczny P, Coolidge DT, Kurzynski TA, Proctor ME, Davis JP. Burkholderia cepacia lower respiratory tract infection associated with exposure to a respiratory therapist. Infect Control Hosp Epidemiol 2001;22:423-426.

358. Reboli AC, Koshinski R, Arias K, Marks-Austin K, Stieritz D, Stull TL. An outbreak of Burkholderia cepacia lower respiratory tract infection associated with contaminated albuterol nebulization solution. Infect Control Hosp Epidemiol 1996;17:741743.

359. Romero-Gomez MP, Quiles-Melero MI, Pena Garcia P, et al. Outbreak of Burkholderia cepacia bacteremia caused by contaminated chlorhexidine in a hemodialysis unit. Infect Control Hosp Epidemiol 2008;29:377-378.

360. Takigawa K, Fujita J, Negayama K, et al. Nosocomial outbreak of Pseudomonas cepacia respiratory infection in immunocompromised patients associated with contaminated nebulizer devices. Kansenshogaku Zasshi 1993;67:1115-1125.

361. Weber DJ, Wilson MB, Rutala WA, Thomann CA. Manual ventilation bags as a source for bacterial colonization of intubated patients. Am Rev Respir Dis 1990;142:892-894.

362. Weems JJ Jr. Nosocomial outbreak of Pseudomonas cepacia associated with contamination of reusable electronic ventilator temperature probes. Infect Control Hosp Epidemiol 1993;14: 583-586.

363. Keen M, Foreman A, Wormald PJ. The clinical significance of nasal irrigation bottle contamination. Laryngoscope 2010;120: 2110-2114.

364. Welch KC, Cohen MB, Doghramji LL, et al. Clinical correlation between irrigation bottle contamination and clinical outcomes in post-functional endoscopic sinus surgery patients. Am J Rhinol Allergy 2009;23:401-404.

365. Koh SJ, Song T, Kang YA, et al. An outbreak of skin and soft 
tissue infection caused by Mycobacterium abscessus following acupuncture. Clin Microbiol Infect 2010;16:895-901.

366. Newman MI, Camberos AE, Clynes ND, Ascherman JA. Outbreak of atypical mycobacteria infections in U.S. patients traveling abroad for cosmetic surgery. Plast Reconstr Surg 2005; 115:964-965.

367. Liu L, Coenye T, Burns JL, Whitby PW, Stull TL, LiPuma JJ. Ribosomal DNA-directed PCR for identification of Achromobacter (Alcaligenes) xylosoxidans recovered from sputum samples from cystic fibrosis patients. J Clin Microbiol 2002;40: $1210-1213$.

368. Kennedy BS, Bedard B, Younge M, et al. Outbreak of Mycobacterium chelonae infection associated with tattoo ink. $N$ Engl J Med 2012;367:1020-1024.

369. Winthrop KL, Abrams M, Yakrus M, et al. An outbreak of mycobacterial furunculosis associated with footbaths at a nail salon. N Engl J Med 2002;346:1366-1371.

370. Haiduven D. Nosocomial aspergillosis and building construction. Med Mycol 2009;47(suppl 1):S210-S216.

371. Vonberg RP, Gastmeier P. Nosocomial aspergillosis in outbreak settings. J Hosp Infect 2006;63:246-254.

372. Weber DJ, Peppercorn A, Miller MB, Sickbert-Benett E, Rutala WA. Preventing healthcare-associated Aspergillus infections: review of recent CDC/HICPAC recommendations. Med Mycol 2009;47(suppl 1):S199-S209.

373. Ashish A, Shaw M, Winstanley C, Humphreys L, Walshaw MJ. Halting the spread of epidemic Pseudomonas aeruginosa in an adult cystic fibrosis centre: a prospective cohort study. JRSM Short Rep 2013;4:1.

374. Festini F, Buzzetti R, Bassi C, et al. Isolation measures for prevention of infection with respiratory pathogens in cystic fibrosis: a systematic review. J Hosp Infect 2006;64:1-6.

375. Griffiths AL, Wurzel DF, Robinson PJ, Carzino R, Massie J. Australian epidemic strain pseudomonas (AES-1) declines further in a cohort segregated cystic fibrosis clinic. J Cyst Fibros 2012;11:49-52.

376. Conway S. Segregation is good for patients with cystic fibrosis. J R Soc Med 2008;101(suppl 1):S31-S35.

377. Resar R, Griffin F, Haraden C, Nolan T. Using Care Bundles to Improve Health Care Quality. Institute for Healthcare Improvement (IHI) Innovation Series white paper. Cambridge, MA: IHI, 2012.

378. Kaier K, Wilson C, Hulscher M, et al. Implementing strategic bundles for infection prevention and management. Infection 2012;40:225-228.

379. Marwick C, Davey P. Care bundles: the holy grail of infectious risk management in hospital? Curr Opin Infect Dis 2009;22: 364-369.

380. Centers for Disease Control and Prevention. Cover Your Cough. http://www.cdc.gov/flu/protect/covercough.htm. Published 2010. Accessed January 13, 2014.

381. Miroballi Y, Garber E, Jia H, et al. Infection control knowledge, attitudes, and practices among cystic fibrosis patients and their families. Pediatr Pulmonol 2012;47:144-152.

382. Saiman L, Garber E. Infection control in cystic fibrosis: barriers to implementation and ideas for improvement. Curr Opin Pulm Med 2009;15:626-631.

383. Cooper T. Putting educational theory into clinical practice. $J$ Hosp Infect 2007;65(suppl 2):124-127.

384. Ferguson PE, Jordens CF, Gilroy NM. Patient and family ed- ucation in HSCT: improving awareness of respiratory virus infection and influenza vaccination. A descriptive study and brief intervention. Bone Marrow Transplant 2010;45:656-661.

385. Larson EL. New rules for the game: interdisciplinary education for health professionals. 1995. Nurs Outlook 2012;60:264-270.

386. Matlow AG, Wray R, Richardson SE. Attitudes and beliefs, not just knowledge, influence the effectiveness of environmental cleaning by environmental service workers. Am J Infect Control 2012;40:260-262.

387. O’Boyle CA, Henly SJ, Larson E. Understanding adherence to hand hygiene recommendations: the theory of planned behavior. Am J Infect Control 2001;29:352-360.

388. Erasmus V, Brouwer W, van Beeck EF, et al. A qualitative exploration of reasons for poor hand hygiene among hospital workers: lack of positive role models and of convincing evidence that hand hygiene prevents cross-infection. Infect Control Hosp Epidemiol 2009;30:415-419.

389. Positive Deviance Initiative. What Is Positive Deviance? http:// www.positivedeviance.org. Published 2010. Accessed January 13, 2014.

390. Marra AR, Guastelli LR, de Araujo CM, et al. Positive deviance: a new strategy for improving hand hygiene compliance. Infect Control Hosp Epidemiol 2010;31:12-20.

391. Hysong SJ, Best RG, Pugh JA. Audit and feedback and clinical practice guideline adherence: making feedback actionable. Implement Sci 2006;1:9.

392. Larson EL, Patel SJ, Evans D, Saiman L. Feedback as a strategy to change behaviour: the devil is in the details. J Eval Clin Pract 2013;19:230-234.

393. Boyce JM, Pittet D; Society for Healthcare Epidemiology of America, Association for Professionals in Infection Control, Infectious Diseases Society of America. Guideline for hand hygiene in health-care settings: recommendations of the Healthcare Infection Control Practices Advisory Committee and the HICPAC/ SHEA/APIC/IDSA Hand Hygiene Task Force. MMWR Recomm Rep 2002;51:1-45.

394. Pittet D, Allegranzi B, Boyce J. The World Health Organization guidelines on hand hygiene in health care and their consensus recommendations. Infect Control Hosp Epidemiol 2009;30:611622.

395. Aiello AE, Coulborn RM, Perez V, Larson EL. Effect of hand hygiene on infectious disease risk in the community setting: a meta-analysis. Am J Public Health 2008;98:1372-1381.

396. Warren-Gash C, Fragaszy E, Hayward AC. Hand hygiene to reduce community transmission of influenza and acute respiratory tract infection: a systematic review. Influenza Other Respir Viruses 2013;7:738-749.

397. Aiello AE, Larson EL, Levy SB. Consumer antibacterial soaps: effective or just risky? Clin Infect Dis 2007;45(suppl 2):S137S147.

398. McGinley KJ, Larson EL, Leyden JJ. Composition and density of microflora in the subungual space of the hand. J Clin Microbiol 1988;26:950-953.

399. Hedderwick SA, McNeil SA, Lyons MJ, Kauffman CA. Pathogenic organisms associated with artificial fingernails worn by healthcare workers. Infect Control Hosp Epidemiol 2000;21:505509.

400. McNeil SA, Foster CL, Hedderwick SA, Kauffman CA. Effect of hand cleansing with antimicrobial soap or alcohol-based gel 
on microbial colonization of artificial fingernails worn by health care workers. Clin Infect Dis 2001;32:367-372.

401. Pottinger J, Burns S, Manske C. Bacterial carriage by artificial versus natural nails. Am J Infect Control 1989;17:340-344.

402. Parry MF, Grant B, Yukna M, et al. Candida osteomyelitis and diskitis after spinal surgery: an outbreak that implicates artificial nail use. Clin Infect Dis 2001;32:352-357.

403. Gordin FM, Schultz ME, Huber R, Zubairi S, Stock F, Kariyil J. A cluster of hemodialysis-related bacteremia linked to artificial fingernails. Infect Control Hosp Epidemiol 2007;28:743744.

404. Moolenaar RL, Crutcher JM, San Joaquin VH, et al. A prolonged outbreak of Pseudomonas aeruginosa in a neonatal intensive care unit: did staff fingernails play a role in disease transmission? Infect Control Hosp Epidemiol 2000;21:80-85.

405. Gupta A, Della-Latta P, Todd B, et al. Outbreak of extendedspectrum $\beta$-lactamase-producing Klebsiella pneumoniae in a neonatal intensive care unit linked to artificial nails. Infect Control Hosp Epidemiol 2004;25:210-215.

406. US Food and Drug Administration. About Personal Protective Equipment (PPE). http://www.fda.gov/MedicalDevices/Products andMedicalProcedures/GeneralHospitalDevicesandSupplies /PersonalProtectiveEquipment/ucm055943.htm. Published 2010. Accessed January 13, 2014.

407. Harris AD, Pineles L, Belton B, et al. Universal glove and gown use and acquisition of antibiotic-resistant bacteria in the ICU: a randomized trial. JAMA 2013;310:1571-1580.

408. Morgan DJ, Liang SY, Smith CL, et al. Frequent multidrugresistant Acinetobacter baumannii contamination of gloves, gowns, and hands of healthcare workers. Infect Control Hosp Epidemiol 2010;31:716-721.

409. Perry C, Marshall R, Jones E. Bacterial contamination of uniforms. J Hosp Infect 2001;48:238-241.

410. Zachary KC, Bayne PS, Morrison VJ, Ford DS, Silver LC, Hooper DC. Contamination of gowns, gloves, and stethoscopes with vancomycin-resistant enterococci. Infect Control Hosp Epidemiol 2001;22:560-564.

411. Bearman GM, Marra AR, Sessler CN, et al. A controlled trial of universal gloving versus contact precautions for preventing the transmission of multidrug-resistant organisms. Am J Infect Control 2007;35:650-655.

412. Tenorio AR, Badri SM, Sahgal NB, et al. Effectiveness of gloves in the prevention of hand carriage of vancomycin-resistant enterococcus species by health care workers after patient care. Clin Infect Dis 2001;32:826-829.

413. Trick WE, Vernon MO, Welbel SF, Demarais P, Hayden MK, Weinstein RA. Multicenter intervention program to increase adherence to hand hygiene recommendations and glove use and to reduce the incidence of antimicrobial resistance. Infect Control Hosp Epidemiol 2007;28:42-49.

414. Gammon J, Morgan-Samuel H, Gould D. A review of the evidence for suboptimal compliance of healthcare practitioners to standard/universal infection control precautions. J Clin Nurs 2008;17:157-167.

415. US Department of Labor. Occupational Safety and Health Administration regulations 1910.134: respiratory protection. https://www.osha.gov/pls/oshaweb/owadisp.show_document $? \mathrm{p} \_$table $=$STANDARDS\&p_id $=12716$. Published 2011. Accessed January 13, 2014.

416. Carling PC, Huang SS. Improving healthcare environmental cleaning and disinfection: current and evolving issues. Infect Control Hosp Epidemiol 2013;34:507-513.

417. Strausbaugh LJ, Siegel JD, Weinstein RA. Preventing transmission of multidrug-resistant bacteria in health care settings: a tale of 2 guidelines. Clin Infect Dis 2006;42:828-835.

418. Passaretti CL, Otter JA, Reich NG, et al. An evaluation of environmental decontamination with hydrogen peroxide vapor for reducing the risk of patient acquisition of multidrugresistant organisms. Clin Infect Dis 2013;56:27-35.

419. Centers for Disease Control and Prevention. Options for Evaluating Environmental Cleaning. http://www.cdc.gov/HAI /toolkits/Evaluating-Environmental-Cleaning.html. Published 2010. Accessed January 13, 2014.

420. Boyce JM, Havill NL, Havill HL, Mangione E, Dumigan DG, Moore BA. Comparison of fluorescent marker systems with 2 quantitative methods of assessing terminal cleaning practices. Infect Control Hosp Epidemiol 2011;32:1187-1193.

421. Rutala WA, Weber DJ. New developments in reprocessing semicritical items. Am J Infect Control 2013;41:S60-S66.

422. Harbarth S, Sudre P, Dharan S, Cadenas M, Pittet D. Outbreak of Enterobacter cloacae related to understaffing, overcrowding, and poor hygiene practices. Infect Control Hosp Epidemiol 1999; 20:598-603.

423. Sheth NK, Post GT, Wisniewski TR, Uttech BV. Multidose vials versus single-dose vials: a study in sterility and cost-effectiveness. J Clin Microbiol 1983;17:377-379.

424. Guidelines for preventing health-care-associated pneumonia, 2003 recommendations of the CDC and the Healthcare Infection Control Practices Advisory Committee. Respir Care 2004; 49:926-939.

425. Ari A, Restrepo RD. Aerosol delivery device selection for spontaneously breathing patients: 2012. Respir Care 2012;57:613626.

426. Lester MK, Flume PA, Gray SL, Anderson D, Bowman CM. Nebulizer use and maintenance by cystic fibrosis patients: a survey study. Respir Care 2004;49:1504-1508.

427. O’Malley CA, VandenBranden SL, Zheng XT, Polito AM, McColley SA. A day in the life of a nebulizer: surveillance for bacterial growth in nebulizer equipment of children with cystic fibrosis in the hospital setting. Respir Care 2007;52:258-262.

428. Hutchinson GR, Parker S, Pryor JA, et al. Home-use nebulizers: a potential primary source of Burkholderia cepacia and other colistin-resistant, gram-negative bacteria in patients with cystic fibrosis. J Clin Microbiol 1996;34:584-587.

429. Jakobsson BM, Onnered AB, Hjelte L, Nystrom B. Low bacterial contamination of nebulizers in home treatment of cystic fibrosis patients. J Hosp Infect 1997;36:201-207.

430. Pitchford KC, Corey M, Highsmith AK, et al. Pseudomonas species contamination of cystic fibrosis patients' home inhalation equipment. J Pediatr 1987;111:212-216.

431. Rosenfeld M, Joy P, Nguyen CD, Krzewinski J, Burns JL. Cleaning home nebulizers used by patients with cystic fibrosis: is rinsing with tap water enough? J Hosp Infect 2001;49:229-230.

432. Blau H, Mussaffi H, Mei Zahav M, et al. Microbial contamination of nebulizers in the home treatment of cystic fibrosis. Child Care Health Dev 2007;33:491-495.

433. Best M, Sattar SA, Springthorpe VS, Kennedy ME. Comparative mycobactericidal efficacy of chemical disinfectants in suspension and carrier tests. Appl Environ Microbiol 1988;54:28562858 . 
434. Merritt K, Hitchins VM, Brown SA. Safety and cleaning of medical materials and devices. J Biomed Mater Res 2000;53: 131-136.

435. Rosaspina S, Salvatorelli G, Anzanel D. The bactericidal effect of microwaves on Mycobacterium bovis dried on scalpel blades. J Hosp Infect 1994;26:45-50.

436. Rosaspina S, Salvatorelli G, Anzanel D, Bovolenta R. Effect of microwave radiation on Candida albicans. Microbios 1994;78: $55-59$.

437. Sanborn MR, Wan SK, Bulard R. Microwave sterilization of plastic tissue culture vessels for reuse. Appl Environ Microbiol 1982;44:960-964.

438. Towle D, Callan DA, Farrel PA, Egan ME, Murray TS. Baby bottle steam sterilizers disinfect home nebulizers inoculated with bacterial respiratory pathogens. J Cyst Fibros 2013;12:512516.

439. Rutala WA, ed. Disinfection, Sterilization and Antisepsis in Health Care. Champlain, NY: Polyscience Publications, 1998: 133-149.

440. Luebbert P. Home care. In: Olmstead R, ed. APIC Text of Infection Control and Epidemiology: Principles and Practice. Washington, DC: Association for Professionals in Infection Control and Epidemiology, 2000:44-47.

441. Karapinar M, Gonul SA. Effects of sodium bicarbonate, vinegar, acetic and citric acids on growth and survival of Yersinia enterocolitica. Int J Food Microbiol 1992;16:343-347.

442. Rutala WA, Barbee SL, Aguiar NC, Sobsey MD, Weber DJ. Antimicrobial activity of home disinfectants and natural products against potential human pathogens. Infect Control Hosp Epidemiol 2000;21:33-38.

443. Reychler G, Leonard A, Van Ossel C, et al. Impact of hypochlorite-based disinfection on bacterial contamination of cystic fibrosis patients' home-nebulisers. J Hosp Infect 2009;72:351357.

444. Jones AM, Dodd ME, Govan JR, et al. Prospective surveillance for Pseudomonas aeruginosa cross-infection at a cystic fibrosis center. Am J Respir Crit Care Med 2005;171:257-260.

445. McKay KO, Cooper PJ, van Asperen PP. Segregation of children with CF diagnosed via newborn screening and acquisition of Pseudomonas aeruginosa. J Cyst Fibros 2009;8:400-404.

446. Wiehlmann L, Cramer N, Ulrich J, Hedtfeld S, Weissbrodt H, Tummler B. Effective prevention of Pseudomonas aeruginosa cross-infection at a cystic fibrosis centre-results of a 10-year prospective study. Int J Med Microbiol 2012;302:69-77.

447. Saiman L. Infection prevention and control in cystic fibrosis. Curr Opin Infect Dis 2011;24:390-395.

448. Ledson MJ, Gallagher MJ, Corkill JE, Hart CA, Walshaw MJ. Cross infection between cystic fibrosis patients colonised with Burkholderia cepacia. Thorax 1998;53:432-436.

449. Zhou J, Garber E, Saiman L. Survey of infection control policies for patients with cystic fibrosis in the United States. Am J Infect Control 2008;36:220-222.

450. Centers for Disease Control and Prevention. Stopping the Spread of Germs at Home, Work, and School. http://www.cdc .gov/flu/protect/stopgerms.htm. Published 2013. Accessed January $13,2014$.

451. Centers for Disease Control and Prevention. Building Air Quality: A Guide for Building Owners and Facility Managers. http:// www.cdc.gov/niosh/docs/91-114/. Published 1991. Accessed January 13, 2014.
452. Rutala WA, Jones SM, Worthington JM, Reist PC, Weber DJ. Efficacy of portable filtration units in reducing aerosolized particles in the size range of Mycobacterium tuberculosis. Infect Control Hosp Epidemiol 1995;16:391-398.

453. Manning ML, Davis J, Sparnon E, Ballard RM. iPads, droids, and bugs: infection prevention for mobile handheld devices at the point of care. Am J Infect Control 2013;41:1073-1076.

454. Guzman-Cottrill JA, Ravin KA, Bryant KA, Zerr DM, Kociolek L, Siegel JD. Infection prevention and control in residential facilities for pediatric patients and their families. Infect Control Hosp Epidemiol 2013;34:1003-1041.

455. Hoogkamp-Korstanje JA, Meis JF, Kissing J, van der Laag J, Melchers WJ. Risk of cross-colonization and infection by Pseudomonas aeruginosa in a holiday camp for cystic fibrosis patients. J Clin Microbiol 1995;33:572-575.

456. Ojeniyi B, Frederiksen B, Hoiby N. Pseudomonas aeruginosa cross-infection among patients with cystic fibrosis during a winter camp. Pediatr Pulmonol 2000;29:177-181.

457. Brimicombe RW, Dijkshoorn L, van der Reijden TJ, et al. Transmission of Pseudomonas aeruginosa in children with cystic fibrosis attending summer camps in the Netherlands. J Cyst Fibros 2008;7:30-36.

458. Centers for Disease Control and Prevention. Influenza Antiviral Medications: Summary for Clinicians. http://www.cdc.gov/flu /professionals/antivirals/summary-clinicians.htm. Published 2013. Accessed January 13, 2014.

459. General recommendations on immunization-recommendations of the Advisory Committee on Immunization Practices (ACIP). MMWR Recomm Rep 2011;60:1-64.

460. Centers for Disease Control and Prevention. Immunization Schedules. http://www.cdc.gov/vaccines/schedules/index.html. Published 2013. Accessed January 13, 2014.

461. Centers for Disease Control and Prevention. Use of 13-valent pneumococcal conjugate vaccine and 23-valent pneumococcal polysaccharide vaccine among children aged 6-18 years with immunocompromising conditions: recommendations of the Advisory Committee on Immunization Practices (ACIP). MMWR Morb Mortal Wkly Rep 2013;62:521-524.

462. Doring G, Pier GB. Vaccines and immunotherapy against Pseudomonas aeruginosa. Vaccine 2008;26:1011-1024.

463. Fowler VG, Allen KB, Moreira ED, et al. Effect of an investigational vaccine for preventing Staphylococcus aureus infections after cardiothoracic surgery: a randomized trial. JAMA 2013;309:1368-1378.

464. Sharma A, Krause A, Worgall S. Recent developments for Pseudomonas vaccines. Hum Vaccin 2011;7:999-1011.

465. Centers for Disease Control and Prevention. Prevention and control of seasonal influenza with vaccines: recommendations of the Advisory Committee on Immunization PracticesUnited States, 2013-2014. MMWR Recomm Rep 2013;62:1-43.

466. Grizas AP, Camenga D, Vazquez M. Cocooning: a concept to protect young children from infectious diseases. Curr Opin Pediatr 2012;24:92-97.

467. Zaman K, Roy E, Arifeen SE, et al. Effectiveness of maternal influenza immunization in mothers and infants. $N$ Engl J Med 2008;359:1555-1564.

468. Eick AA, Uyeki TM, Klimov A, et al. Maternal influenza vaccination and effect on influenza virus infection in young infants. Arch Pediatr Adolesc Med 2011;165:104-111.

469. Rubin LG, Levin MJ, Ljungman P, et al. 2013 IDSA clinical 
practice guideline for vaccination of the immunocompromised host. Clin Infect Dis 2014;58:309-318.

470. Saldana PS, Pomeranz JL. Cystic fibrosis and the workplace: a review of the literature. Work 2012;42:185-193.

471. Cystic Fibrosis Foundation. Cystic Fibrosis in the Workplace. http://www.cff.org/UploadedFiles/LivingWithCF/InWorkplace /CFInTheWorkplace.pdf. Published 2003. Accessed January 13, 2014.

472. Tuchman LK, Schwartz LA, Sawicki GS, Britto MT. Cystic fibrosis and transition to adult medical care. Pediatrics 2010;125: $566-573$.

473. Al-Yateem N. Guidelines for the transition from child to adult cystic fibrosis care. Nurs Child Young People 2013;25:29-34.

474. Tuchman L, Schwartz M. Health outcomes associated with transition from pediatric to adult cystic fibrosis care. Pediatrics 2013;132:847-853.

475. Walters S. Association of Cystic Fibrosis Adults Survey 1994. London: CF Trust, 1995.

476. Walters S. Health service careers for people with cystic fibrosis. J R Soc Med 2002;95(suppl 41):41-51.

477. David TJ. Can patients with cystic fibrosis have a career in the health service? J R Soc Med 2002;95:278-279.

478. US Department of Health and Human Services. Understanding Health Information Privacy. http://www.hhs.gov/ocr/privacy /hipaa/understanding/. Accessed January 13, 2014.

479. Barratt RL, Shaban R, Moyle W. Patient experience of source isolation: lessons for clinical practice. Contemp Nurse 2011;39: 180-193.

480. Chua SE, Cheung V, McAlonan GM, et al. Stress and psychological impact on SARS patients during the outbreak. Can J Psychiatry 2004;49:385-390.

481. Davies H, Rees J. Psychological effects of isolation nursing (1): mood disturbance. Nurs Stand 2000;14:35-38.

482. Stajduhar KI, Neithercut J, Chu E, et al. Thyroid cancer: patients' experiences of receiving iodine-131 therapy. Oncol Nurs Forum 2000;27:1213-1218.

483. Catalano G, Houston SH, Catalano MC, et al. Anxiety and depression in hospitalized patients in resistant organism isolation. South Med J 2003;96:141-145.

484. Wassenberg MW, Severs D, Bonten MJ. Psychological impact of short-term isolation measures in hospitalised patients. J Hosp Infect 2010;75:124-127.

485. Stelfox HT, Bates DW, Redelmeier DA. Safety of patients isolated for infection control. JAMA 2003;290:1899-1905.

486. Abad C, Fearday A, Safdar N. Adverse effects of isolation in hospitalised patients: a systematic review. J Hosp Infect 2010; 76:97-102.

487. Morgan DJ, Diekema DJ, Sepkowitz K, Perencevich EN. Adverse outcomes associated with contact precautions: a review of the literature. Am J Infect Control 2009;37:85-93.

488. Day HR, Perencevich EN, Harris AD, et al. Depression, anxiety, and moods of hospitalized patients under contact precautions. Infect Control Hosp Epidemiol 2013;34:251-258.

489. Russo K, Donnelly M, Reid AJ. Segregation-the perspectives of young patients and their parents. J Cyst Fibros 2006;5:9399.

490. Griffiths AL, Armstrong D, Carzino R, Robinson P. Cystic fibrosis patients and families support cross-infection measures. Eur Respir J 2004;24:449-452.

491. Rees J, Davies HR, Birchall C, Price J. Psychological effects of source isolation nursing (2): patient satisfaction. Nurs Stand 2000;14:32-36.

492. Ward D. Ageism and the abuse of older people in health and social care. Br J Nurs 2000;9:560-563.

493. Gammon J. Analysis of the stressful effects of hospitalisation and source isolation on coping and psychological constructs. Int J Nurs Pract 1998;4:84-96.

494. Campbell T. Feelings of oncology patients about being nursed in protective isolation as a consequence of cancer chemotherapy treatment. J Adv Nurs 1999;30:439-447.

495. Gammon J. The psychological consequences of source isolation: a review of the literature. J Clin Nurs 1999;8:13-21.

496. Ferrin M, Robinson C, Hadjiliadis D, Holsclaw DS. Internet technology use by adults for socialization: impact of CF Foundation infection control guidelines. Pediatr Pulmonol 2010; 46(suppl 33):445.

497. Marciel KK, Saiman L, Quittell LM, Dawkins K, Quittner AL. Cell phone intervention to improve adherence: cystic fibrosis care team, patient, and parent perspectives. Pediatr Pulmonol 2010;45:157-164.

498. Cabana MD, Rand CS, Powe NR, et al. Why don't physicians follow clinical practice guidelines? a framework for improvement. JAMA 1999;282:1458-1465.

499. Garber E, Desai M, Zhou J, et al. Barriers to adherence to cystic fibrosis infection control guidelines. Pediatr Pulmonol 2008;43: 900-907.

500. Davis DA, Thomson MA, Oxman AD, Haynes RB. Changing physician performance: a systematic review of the effect of continuing medical education strategies. JAMA 1995;274:700705. 